\title{
EXPLICIT HARMONIC STRUCTURE OF BIDIMENSIONAL LINEAR STRAIN-GRADIENT ELASTICITY
}

\author{
N. AUFFRAY, H. ABDOUL-ANZIZ, AND B. DESMORAT
}

\begin{abstract}
In the perspective of homogenization theory, strain-gradient elasticity is a strategy to describe the overall behaviour of materials with coarse mesostructure. In this approach, the effect of the mesostructure is described by the use of three elasticity tensors whose orders vary from 4 to 6 . Higher-order constitutive tensors make it possible to describe rich physical phenomena. However, these objects have intricate algebraic structures that prevent us from having a clear picture of their modeling capabilities. The harmonic decomposition is a fundamental tool to investigate the anisotropic properties of constitutive tensor spaces. For higher-order tensors (i.e. tensors of order $n \geq 3$ ), their determination is generally a difficult task. In this paper, a novel procedure to obtain this decomposition is introduced. This method, which we have called the Clebsch-Gordan Harmonic Algorithm, allows one to obtain explicit harmonic decompositions satisfying good properties such as orthogonality and uniqueness. The elements of the decomposition also have a precise geometrical meaning simplifying their physical interpretation. This new algorithm is here developed in the specific case of 2D space and applied to Mindlin's Strain-Gradient Elasticity. We provide, for the first time, the harmonic decompositions of the fifth- and sixth-order elasticity tensors involved in this constitutive law. The Clebsch-Gordan Harmonic Algorithm is not restricted to strain-gradient elasticity and may find interesting applications in different fields of mechanics which involve higher-order tensors.
\end{abstract}

\section{INTRODUCTION}

Strain-Gradient Elasticity. Continuum mechanics is a well-established theory which constitutes the classical framework to study strain and stress in solid materials. The physics contained in the theory is versatile enough to describe the inner state of a planet subjected to the force of gravity, as well as to meet the daily needs of a mechanical engineer. These successes make classical continuum mechanics a fundamental theory of modern physics. Nevertheless, despite all its successes, situations have arisen in which its classical formulation reaches its limits and fails to correctly describe the physics at work: mechanics of nano-structures [81, 31, 22], elastic waves in periodic continua [28,61], capillarity and surface tension phenomena [20,53,65, 35], etc. Despite their diversities, these examples have in common that they show dependencies to characteristic lengths, a property that cannot be taken into account within the classical formulation of continuum mechanics.

Since the pioneering work of the Cosserat brothers in the early years of the 20th century [23], many scientists have proposed enriched continuum theories to extend the capabilities of the standard theory. With the contributions of Koiter [48], Toupin [71], Eringen [32], Mindlin [52, 53, 54] and many others, the 60' was probably the most fertile period of this project [50]. At that time, the community of theoretical mechanics developed many models that are still relevant today. Leaving aside non-local aspects, the classical theory can be enriched either by including additional degrees of freedom (DOF) or by including higher-gradients of the DOF in the mechanical energy. All these models suffer the drawbacks: 1) to require too many material parameters to be of practical uses and 2) due to the intrinsic complexity of the equations, the content of the physics described by these models is difficult to grasp. At that time, except for some applications in physics to describe the dispersion of elastic waves in crystals [28], or to describe the mechanics of liquid crystals [33], these models were mostly unused.

The development of numerical homogenization methods that allow the coefficients required by the extended models to be related to a known mesostructure has changed the situation [34, 11, 72]. There is now a renewed interest in generalized continuum theories $[75,5,12,15]$. This renewed interest has also been

Date: December 22, 2020.

2010 Mathematics Subject Classification. 74B05; 74E10.

Key words and phrases. Anisotropy; Symmetry classes; Higher-order tensors; Harmonic decomposition. 
supported by the emergence of additive manufacturing techniques that permit specific mesostructures to be fabricated (almost) on demand [77, 2, 42]. Recently theoretical approaches have been developed to design architectured materials in order to maximise the non-standard effects predicted by generalized mechanics [13], and for the first time the associated materials have been produced and tested [57].

In statics, to have an important contribution in the overall behaviour of strain-gradient effects, the classical elasticity needs to be almost degenerated [1, 44]. This aspect has been widely studied in pantographic structures [3, 25], and is also encountered in pentamode metamaterials [51, 46] which also possess quasidegenerated deformation modes. In dynamics, the contribution of higher-order effects is easier to highlight and to control $[6,12,18]$. In some recent contributions $[63,62]$, it has been shown that for honeycomb materials, higher-order effects make a significant contribution as soon as the wavelength is about 10 times the size of unit cell size. This effect has been used in [62] to bend the trajectory of an elastic wave around a circular hole. The control of this effect in more general situations can find interesting engineering applications [19, 64].

The design of architectured materials in which these higher-order effects are maximised, or at the contrary inhibited, is at the present time an open and challenging problem in mechanical sciences. An approach to the optimal design of strain-gradient architectured materials is to use topological optimisation algorithms $[24,60,4,26]$. For isotropic continua the material optimisation process is formulated by expressing the design functionals as functions of the isotropic parameters of the different constitutive tensors[14]. For anisotropic materials the previous approach cannot be extended without a few precautions. A path to this rigorous extension is through the use of tensor invariants [60, 59].

Harmonic decomposition. To optimize the material independently of its spatial orientation, design functionals should be expressed as functions of tensor invariants rather than in terms of tensor components. The associated mathematical theory is the invariant theory, which aims at determining the minimal number of tensorial functions that are invariant with respect to a given group of transformations. Here, since a material is left invariant by orthogonal transformations (rotations and improper transformations), the group of transformations will be $\mathrm{O}(d)$ in a $d$-dimensional space. If the mathematical theory is clear in any dimension, its practical and effective application strongly depend on the space dimension. For $d=2$ the situation is rather clear and general results are available [79,27], while the case $d=3$ presents some serious difficulties preventing general results $[17,66,56,21]$.

In both cases, the effective construction of a basis of invariants lies on a first step which is the determination of an explicit irreducible decomposition of the constitutive tensors. In the case of a symmetric second-order tensor, this decomposition corresponds to the decomposition of a tensor into a spherical and a deviatoric part. This approach can be generalized to tensors of arbitrary order [45, 79, 9]. If the formal structure of these decompositions is easy to determine, both in $2 \mathrm{D}$ and $3 \mathrm{D}$, when it comes to determining an explicit decomposition formula, things become more difficult:

- even if the harmonic structure is uniquely defined, the explicit decomposition is, in general, not unique and hence different, albeit isomorphic, constructions are possible [38];

- without a pertinent choice for the explicit harmonic decomposition, the derived tensor invariants will not have a clear physical content;

- the complexity of the computations increases quickly with the tensor order. An algorithmic procedure is, therefore, mandatory to perform this decomposition.

In the present study, which is devoted to bidimensional strain-gradient elasticity, the constitutive law involves tensors of order ranging from 4 to 6 . If the harmonic decomposition of the fourth-order elasticity tensor is well known [76, 26], for the fifth- and sixth-order tensors no decompositions are, to the best of the authors' knowledge, available in the literature. Some procedures to perform this decomposition are available in the literature of continuum mechanics:

- Spencer's Algorithm [68]: Spencer's method consists in first reducing a general tensor into totally symmetric tensors and then decomposing each totally symmetric tensor into totally symmetric traceless tensors. It is, at the present time, the most known and used algorithm [36, 37, 49]. However, this approach suffers the following limitations:

- the treatment of higher-order tensors is quickly intractable;

- its numerical implementation is not straightforward. 
If efficient in simple situations, the Spencer's algorithm seems to be of limited interest to treat more complicated problems.

- Verchery's Method [74, 73]: Verchery's approach is based on a complex change of variable, in a sense analogous to the transformation of Green and Zerna [40], which can also be rooted to the work of Kolosov and Muskhelishvili [55]. This method, which was recently re-explored in [27], is elegant, but its main limitations are:

- it does not produce a practical formula for the decomposition;

- when different harmonic terms of the same order are present, the pairing of their components is not direct;

- the method is restricted to the $2 \mathrm{D}$ cases.

- Zou's Approach [80, 82]: The Zou's method exploits a Clebsch-Gordan identity to construct an orthogonal harmonic decomposition of a $n$ th-order tensor from the orthogonal harmonic decomposition of a $(n-1)$ th-order tensor. This iterative method is powerful to obtain orthogonal harmonic decomposition of higher-order tensors without index symmetry. Its application to higher-order tensors having specific index symmetries is possible, but can be cumbersome.

In addition, as a common limitation, none of the three listed methods provides a clear mechanical content to the harmonic tensors of the resulting decomposition.

The objective of the present contribution is to introduce a new method for determining explicit harmonic decompositions that solves the problems identified with the previous methods. The algorithm we propose, which will be referred to as the Clebsch-Gordan Harmonic Algorithm, will be conducted here in a 2D framework, but it can be extended without any conceptual obstruction to the 3D framework.

The main idea of the Clebsch-Gordan approach, and the main difference from all other methods, is first to decompose not the constitutive tensor (e.g. the fourth-order elasticity tensor for classical elasticity) but the state tensors on which it acts (e.g. the strain/stress second-order tensor). This first decomposition will induce a block structure on the constitutive tensor. If the elementary blocks are generally not harmonic, their harmonic structures are very simple, and their decomposition into irreducible tensors easy to proceed. The combination of these different steps leads to an explicit harmonic decomposition of the constitutive tensor. An example of this situation is provided in Appendix C. Compared to the other methods, the main advantages of the proposed approach are:

(1) the procedure is algorithmic and can easily be implemented in a Computer Algebra System;

(2) once a decomposition of the state tensor spaces chosen, the resulting decomposition of the constitutive tensors is uniquely defined;

(3) the elements of the decomposition are orthogonal to each other and have a clear physical content.

Further, since the space of state tensors is first decomposed, the resulting harmonic decomposition implies a decomposition of the internal energy density. Such a property is valuable to provide a physical content to the higher-order constitutive parameters of the model.

Before closing this introduction, it is important to mention that the proposed method is introduced here in the context of strain-gradient elasticity only because of the authors' interest in this application. Tensors of orders greater than two are necessary in many areas of mechanics. They appear, for example, in generalised continuum theories such as in strain-gradient elasticity [52] or micromorphic elasticity [32], but also in higher-order models of the plastic yielding function [39], for the study of the acoustoelastic effect [70], in continuum damage mechanics in which fabric tensors are involved [47] and, of course, for elastic shells [30]. We believe that our approach to harmonic decomposition can find interesting applications in these different fields.

This article is organized as follows. In Section 1, we introduce notations that we will use throughout the text. Section 2 is devoted to the description of the strain-gradient elasticity constitutive law. By the end of this section, the harmonic structure of the model is introduced and detailed. Section 3 is devoted to the theoretical aspects of the method. Some general results that will be used all along the paper are introduced. In Section 4 the method is detailed for the fourth-order elasticity tensor. The purpose of this section is mainly illustrative and aims a recovering the well-known harmonic decomposition of the elasticity tensor. This approach is then extended, in Section 5, to the decomposition of the fifth- and sixth-order elasticity tensors involved in the model. Those results are, we believe, new and not available in the literature. 


\section{Notations}

Throughout this paper, the two-dimensional Euclidean space $\mathcal{E}^{2}$ is equipped with a rectangular Cartesian coordinate system with origin $\mathrm{O}$ and an orthonormal basis $\mathcal{B}=\left\{\underline{\mathrm{e}}_{1}, \underline{\mathrm{e}}_{2}\right\}$. Upon the choice of a reference point $\mathrm{O}$ in $\mathcal{E}^{2}$ and a given basis $\mathcal{B}, \mathcal{E}^{2}$ will be identified with the 2 -dimensional vector space $\mathbb{R}^{2}$. As a consequence, points in $\mathcal{E}^{2}$ will be designated by their vector positions with respect to $\mathrm{O}$. In the following, $\underline{\mathrm{x}}=\left(x_{1}, x_{2}\right)=x_{1} \underline{\mathrm{e}}_{1}+x_{2} \underline{\mathrm{e}}_{2}=x_{i} \underline{\mathrm{e}}_{i}$, where Einstein summation convention is used, i.e. when an index appears twice in an expression it implies summation of this term over all the values of the index. Below are provided some specific notations and conventions used in this article.

Groups:

- $\mathrm{O}(2)$ : the group of invertible transformations of $\mathbb{R}^{2}$ satisfying $\mathbf{g}^{-1}=\mathbf{g}^{T}$, where $\mathbf{g}^{-1}$ and $\mathbf{g}^{T}$ stand for the inverse and the transpose of $\mathbf{g}$. This group is called the orthogonal group;

- $\mathrm{SO}(2)$ : the subgroup of $\mathrm{O}(2)$ of elements with determinant 1 , called the special orthogonal group;

- $\mathfrak{S}_{n}$ : the group of all permutations on the set $\{1,2, \ldots, n\}$, called the symmetric group;

- $\mathbb{1}$ : the trivial group solely containing the identity.

As a matrix group, $\mathrm{O}(2)$ is generated by

$$
\mathbf{r}(\theta)=\left(\begin{array}{cc}
\cos \theta & -\sin \theta \\
\sin \theta & \cos \theta
\end{array}\right) \quad \text { with } 0 \leq \theta<2 \pi \quad \text { and } \quad \boldsymbol{\pi}\left(\underline{\mathrm{e}}_{2}\right)=\left(\begin{array}{cc}
1 & 0 \\
0 & -1
\end{array}\right)
$$

where $\mathbf{r}(\theta)$ is the rotation by an angle $\theta$ and $\boldsymbol{\pi}(\underline{\mathrm{n}})$ is the reflection across the line normal to $\underline{\mathrm{n}}$ :

$$
\pi(\underline{\mathrm{n}}):={\underset{\sim}{\mathrm{i}}}^{(2)}-2 \underline{\mathrm{n}} \otimes \underline{\mathrm{n}}, \quad\|\underline{\mathrm{n}}\|=1,
$$

with ${\underset{\sim}{(}}^{(2)}$ the second order identity tensor, as defined below.

To describe the symmetry classes of tensors, the following finite subgroups of $\mathrm{O}(2)$ will be used:

- $\mathrm{Z}_{k}(k \geq 2)$ the cyclic group with $k$ elements, generated by $\mathbf{r}(2 \pi / k)$. For $k=1$, we have $\mathrm{Z}_{k}=\mathbb{1}$;

- $\mathrm{D}_{k}(k \geq 2)$ the dihedral group with $2 k$ elements, generated by $\mathbf{r}(2 \pi / k)$ and $\boldsymbol{\pi}(\underline{\mathrm{n}})$. For $k=1$, the group $\mathrm{D}_{1}^{\underline{\mathrm{n}}}$ will be denoted by $\mathrm{Z}_{2}^{\pi, \underline{\mathrm{n}}}$.

It has to be noted that, up to conjugacy by an element of $\mathrm{SO}(2)$, any $\mathrm{D}_{k}^{\mathrm{n}}$ is conjugate to $\mathrm{D}_{k}^{\mathrm{e}_{2}}$, and will simply be denoted by $\mathrm{D}_{k}$. Accordingly, $\mathrm{Z}_{2}^{\pi, \underline{\mathrm{e}}_{2}}$ will simply be denoted $\mathrm{Z}_{2}^{\pi}$.

Tensor products:

- $\otimes$ denotes the usual tensor product of two tensors or vector spaces;

- $\stackrel{n}{\otimes}$ denotes the $n$th power of the tensor product, for example $\stackrel{n}{\otimes} V:=V \otimes \cdots \otimes V(n$ copies of $V)$;

- $\otimes^{s}$ denotes the symmetrized tensor product;

- $\bar{\otimes}$ and $\underline{\otimes}$ indicate the following partial twisted tensor products between second-order tensors:

$$
(\underset{\sim}{\mathrm{a}} \bar{\otimes} \underset{\sim}{\mathrm{b}})_{i j k l}=a_{i k} b_{j l},(\underset{\sim}{\mathrm{a}} \underset{\sim}{\otimes} \underset{\sim}{\mathrm{b}})_{i j k l}=a_{i l} b_{j k}
$$

- $\underline{\otimes}$ indicates the twisted tensor product defined by

$$
(\underset{\sim}{\mathrm{a}} \underset{\bar{\otimes}}{\mathrm{b}})=\frac{1}{2}(\underset{\sim}{\mathrm{a}} \bar{\otimes} \underset{\sim}{\mathrm{b}}+\underset{\sim}{\mathrm{a}} \underset{\sim}{\otimes} \underset{\sim}{b}) \text {. }
$$

Tensor spaces:

- $\mathbb{G}^{n}:=\stackrel{n}{\otimes} \mathbb{R}^{2}$ is the space of $n$ th-order tensors having no index symmetries;

- $\mathbb{T}^{n}$ is a subspace of $\mathbb{G}^{n}$ defined by its index symmetries;

- $\mathbb{S}^{n}$ is the space of totally symmetric ${ }^{1} n$ th-order tensors on $\mathbb{R}^{2}$;

- $\mathbb{K}^{n}$ is the space of $n$ th-order harmonic tensors (i.e. totally symmetric and traceless tensors), with

$$
\operatorname{dim}\left(\mathbb{K}^{n}\right)= \begin{cases}2 & \text { if } n>0, \\ 1 & \text { if } n \in\{0,-1\}\end{cases}
$$

Among them:

$-\mathbb{K}^{0}$ is the space of scalars;

\footnotetext{
${ }^{1}$ By totally symmetric we mean symmetric with respect to all permutations of indices.
} 
$-\mathbb{K}^{-1}$ is the space of pseudo-scalars (i.e. scalars which change sign under improper transformations).

We will use tensors of different orders, tensors of order $-1,0,1,2,3,4,5$ and 6 are denoted by $\beta, \alpha, \underset{\sim}{\mathrm{v}}, \underset{\sim}{\mathrm{a}}$, $\underset{\simeq}{\mathrm{A}}, \underset{\approx}{\mathrm{B}}, \underset{\approx}{\mathrm{C}}, \underset{\approx}{\mathrm{D}}$, respectively. General tensors (i.e. with no mention of their order) are denoted using bold fonts, as for instance $\mathbf{T}$. With respect to $\mathcal{B}$, the components of $\mathbf{T} \in \mathbb{T}^{n}$ are denoted as

$$
\mathbf{T}=T_{i_{1} \ldots i_{n}} .
$$

The simple, double, triple and fourth-order contractions are written ., :, :, ::, respectively. Generic $k$ th-order contraction will be indicated by the notation ${ }^{(k)}$. In components with respect to $\mathcal{B}$, for general tensors $\mathbf{A}$ and $\mathbf{B}$, these notations correspond to

$$
\begin{gathered}
(\mathbf{A} \cdot \mathbf{B})_{i_{1} \ldots i_{n}}=A_{i_{1} \ldots i_{p} j} B_{j i_{p+1} \ldots i_{n}}, \quad(\mathbf{A}: \mathbf{B})_{i_{1} \ldots i_{n}}=A_{i_{1} \ldots i_{p} j k} B_{j k i_{p+1} \ldots i_{n}}, \\
(\mathbf{A}: \mathbf{B})_{i_{1} \ldots i_{n}}=A_{i_{1} \ldots i_{p} j k l} B_{j k l i_{p+1} \ldots i_{n}}, \quad(\mathbf{A}:: \mathbf{B})_{i_{1} \ldots i_{n}}=A_{i_{1} \ldots i_{p} j k l m} B_{j k l m i_{p+1} \ldots i_{n}} .
\end{gathered}
$$

When needed, index symmetries of both spaces and their elements are expressed as follows: (..) indicates invariance under permutations of the indices in parentheses and.... indicates symmetry with respect to permutations of the underlined blocks. For example, $\underset{\sim}{\mathrm{a}} \in \mathbb{T}_{(i j)}$ means that $a_{i j}=a_{j i}$.

Actions on tensors. We consider the action of two groups on the space $\mathbb{T}^{n}$ :

- the action of $\mathrm{O}(2)$, given by

$$
\forall g \in \mathrm{O}(2), \mathbf{T}^{g}:=g \stackrel{n}{\star} \mathbf{T}=g_{i_{1} j_{1}} g_{i_{2} j_{2}} \ldots g_{i_{n} j_{n}} T_{j_{1} j_{2} \ldots j_{n}} \underline{\mathrm{e}}_{i_{1}} \otimes \ldots \otimes \underline{\mathrm{e}}_{i_{n}} .
$$

This action is the tensorial action, sometimes also known as the Rayleigh product. When clear from the context, no mention will be made of the tensor order in the product, in this case the notation $n_{\star}^{n}$ simplifies to $\star$. The set $\mathrm{G}(\mathbf{T}):=\{g \in \mathrm{O}(2) \mid g \star \mathbf{T}=\mathbf{T}\}$ is called the spatial symmetry group of $\mathbf{T}$. A tensor $\mathbf{T}$ is said to be isotropic if $\mathrm{G}(\mathbf{T})=\mathrm{O}(2)$;

- the action of $\mathfrak{S}_{n}$, given by

$$
\forall \varsigma \in \mathfrak{S}_{n}, \mathbf{T}^{\varsigma}:=\varsigma * \mathbf{T}=T_{i_{\varsigma(1)} i_{\varsigma(2)} \ldots i_{\varsigma(n)}} \underline{\mathrm{e}}_{i_{1}} \otimes \ldots \otimes \underline{\mathrm{e}}_{i_{n}} .
$$

The set $\mathcal{G}(\mathbf{T}):=\left\{\varsigma \in \mathfrak{S}_{n} \mid \varsigma * \mathbf{T}=\mathbf{T}\right\}$ is called the index symmetry group of $\mathbf{T}$. A tensor $\mathbf{T}$ is said to be

- generic if $\mathcal{G}(\mathbf{T})=\mathbb{1}$, elements of $\mathbb{G}^{n}$ verify this property;

- totally symmetric if $\mathcal{G}(\mathbf{T})=\mathfrak{S}_{n}$, elements of $\mathbb{S}^{n}$ verify this property.

The notation $\mathcal{G}\left(\mathbb{T}^{n}\right)$ will also be used to indicate the index symmetry group of a generic element $\mathbf{T} \in \mathbb{T}^{n}$.

Special tensors:

- $\mathbf{I}^{\mathbb{V}}$ is the identity tensor on the vector space $\mathbb{V}$. Identity tensors can be expressed using isotropic tensors [78] :

- $\underset{\sim}{\mathrm{I}^{2}}$ is the second-order identity tensor on $\mathbb{R}^{2}$. It is defined from $\underset{\sim}{\mathrm{i}}{ }^{(2)}$ which components are given by the Kronecker delta $\delta_{i j}$ :

$$
{\stackrel{\mathrm{I}}{\mathbb{R}^{2}}}^{\mathrm{i}_{\sim}^{(2)}}
$$

$-{\underset{\sim}{\mathrm{I}}}^{\mathbb{T}^{(i j)}}={\underset{\sim}{\mathrm{i}}}^{(2)} \underline{\bar{\otimes}} \underset{\sim}{\mathrm{i}}{ }^{(2)}$ is the fourth-order identity tensor on $\mathbb{S}^{2}$.

- $\underset{\sim}{\epsilon}$ denotes the $2 \mathrm{D}$ Levi-Civita tensor defined by

$$
\epsilon_{i j}= \begin{cases}1 & \text { if } i j=12 \\ -1 & \text { if } i j=21 \\ 0 & \text { if } i=j\end{cases}
$$


Miscellaneous notations:

- $\simeq$ denotes an isomorphism;

- $\mathcal{L}(\mathbb{E}, \mathbb{F})$ indicates the space of linear maps from $\mathbb{E}$ to $\mathbb{F}$;

- $\mathcal{L}(\mathbb{E})$ indicates the space of linear maps from $\mathbb{E}$ to $\mathbb{E}$;

- $\mathcal{L}^{s}(\mathbb{E})$ indicates the space of self-adjoint linear maps on $\mathbb{E}$.

Tensor isotropic basis. Let us introduce $\mathbb{I}^{n}$ the space of $n$ th-order isotropic tensors:

$$
\mathbb{I}^{n}:=\left\{\mathbf{T} \in \mathbb{T}^{n} \mid \quad \forall g \in \mathrm{O}(2), \quad g \star \mathbf{T}=\mathbf{T}\right\} .
$$

Elements of $\mathbb{I}^{n}$ are denoted $\mathbf{i}_{p}^{(n)}$, in which $n$ indicates the order of the tensor and $p$ distinguishes among the different isotropic tensors of the same order. Every isotropic tensor can be expressed as a linear combination of products of $\underset{\sim}{i(2)}[78]$. Products of $\underset{\sim}{i(2)}$ will be referred to as elementary isotropic tensors and, by definition, the element $\mathbf{i}_{1}^{(2 p)}$ is defined as:

$$
\mathbf{i}_{1}^{(2 p)}={\underset{\sim}{\mathrm{i}}}^{(2)} \otimes \ldots \otimes{\underset{\sim}{\mathrm{i}}}^{(2)}={\underset{\sim}{\mathrm{i}}}^{(2)} \stackrel{p-1}{\otimes}{\underset{\sim}{\mathrm{i}}}^{(2)}
$$

For fourth-order tensors, there exists 3 elementary isotropic tensors ${ }^{2}$ :

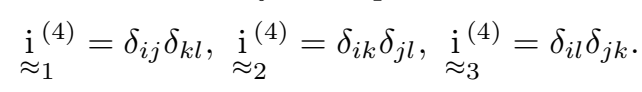

For latter use, this allows us to express the identity tensor on second-order symmetric tensors as

$$
{\underset{\approx}{\mathrm{T}}}^{\mathrm{T}}(i j)=\frac{1}{2}\left(\underset{\approx_{2}}{\mathrm{i}^{(4)}}+\underset{\mathrm{\sim}_{3}^{\mathrm{i}}}{(4)}\right)
$$

For sixth-order tensors, there exists 15 elementary isotropic tensors:

$$
\begin{aligned}
& \stackrel{\mathrm{i}}{(}_{1}^{(6)}=\delta_{i j} \delta_{k l} \delta_{m n}, \quad \stackrel{\mathrm{i}}{(6)}^{(6)}=\delta_{i j} \delta_{k m} \delta_{l n}, \quad{\stackrel{\mathrm{i}}{\approx_{3}}}^{(6)}=\delta_{i j} \delta_{k n} \delta_{l m} \\
& \stackrel{\mathrm{i}}{(6)}_{4}^{(6)}=\delta_{i k} \delta_{j l} \delta_{m n}, \quad{\stackrel{\mathrm{i}}{\mathrm{\sigma}_{5}}}^{(6)}=\delta_{i k} \delta_{j m} \delta_{l n}, \quad{\stackrel{\mathrm{i}}{\mathrm{\sigma}_{6}}}_{6}^{(6)}=\delta_{i k} \delta_{j n} \delta_{l m} \\
& {\stackrel{\mathrm{i}}{\approx_{7}}}^{(6)}=\delta_{i l} \delta_{j k} \delta_{m n}, \quad{\stackrel{\mathrm{i}}{\mathrm{\sigma}_{8}}}^{(6)}=\delta_{i l} \delta_{j m} \delta_{k n}, \quad{\stackrel{\mathrm{i}}{\mathrm{\sigma}_{9}}}_{9}^{(6)}=\delta_{i l} \delta_{j n} \delta_{k m} \\
& \stackrel{\mathrm{i}}{(6)}_{10}^{(6)} \delta_{i m} \delta_{j k} \delta_{l n}, \quad \stackrel{\mathrm{i}}{(6)}^{(6)}=\delta_{i m} \delta_{j n} \delta_{k l}, \quad{\stackrel{\mathrm{i}}{\approx_{12}}}^{(6)}=\delta_{i m} \delta_{j l} \delta_{k n} \\
& {\stackrel{\mathrm{i}}{\mathrm{i}_{13}}}_{(6)}^{(6)}=\delta_{i n} \delta_{j k} \delta_{l m}, \quad{\stackrel{\mathrm{i}}{\approx_{14}}}_{14}^{(6)}=\delta_{i n} \delta_{j l} \delta_{k m}, \quad{\stackrel{\mathrm{i}}{\approx_{15}}}^{(6)}=\delta_{i n} \delta_{j m} \delta_{k l} .
\end{aligned}
$$

According to the dimension of the physical spaces, these elementary tensors may not be necessarily independent. According to Racah [58], in 2D the number of independent fourth-order isotropic tensors is still 3, while for sixth-order isotropic tensors only 10 are independent.

\section{StRAIN-GRADIENT LINEAR ELASTICITY}

We introduce in this section the constitutive law of a linear strain-gradient elastic material [52, 54]. First, we present the state and constitutive tensors of the model. And, in the second part of the section, we detail their harmonic structures, a step mandatory for constructing the harmonic decomposition.

2.1. Constitutive equations. State tensors describe point-wisely the different physical fields (primal and dual) of the model. A linear constitutive law can be viewed as a linear map between the state tensors that characterize a chosen physical model. It is defined by a set of constitutive tensors which describe the influence of the matter on these state tensor fields. More precisely, they describe how primal and dual fields are connected by the matter [69].

In the case of classical elasticity, the state tensors are $\underset{\sim}{\sigma}$ and $\underset{\sim}{\varepsilon}$. These tensors characterize the local state of stress and of strain, respectively ${ }^{3}$, and belong to the same space $\mathbb{T}_{(i j)}$. The linearity of the model implies

\footnotetext{
2 with a slight abuse of notation.

${ }^{3}$ In the infinitesimal setting, the strain tensor is defined from the displacement field $\underline{\mathrm{u}}$ as $\underset{\sim}{\varepsilon}:=\frac{1}{2}(\underline{\mathrm{u}} \otimes \underline{\nabla}+\underline{\nabla} \otimes \underline{\mathrm{u}})$, where $\underline{\nabla}$ denotes the nabla differential operator.
} 
the use of a fourth-order tensor $\underset{\approx}{\mathrm{C}}$ as a constitutive tensor. This tensor can be viewed as an element of $\mathcal{L}^{s}\left(\mathbb{T}_{(i j)}, \mathbb{T}_{(i j)}\right)$. In summary, for classical elasticity:

- State tensors: $\underset{\sim}{\sigma}, \underset{\sim}{\varepsilon}$;

- Constitutive tensor: $\underset{\approx}{\mathrm{C}}$.

The linear strain-gradient elasticity model $[53,54]$ is obtained by extending the set of state tensors by including the strain-gradient tensor $\underset{\simeq}{\eta}:=\underset{\sim}{\varepsilon} \otimes \underline{\nabla}$ and its dual quantity, the hyperstress tensor $\underset{\simeq}{\tau}$. This construction corresponds to the Mindlin's Type II formulation [54]. Those tensors are elements of $\mathbb{T}_{(i j) k}$. The constitutive equations of the model define the stress tensor $\sigma$ and the hyperstress tensor $\underset{\sim}{\tau}$ as linear functions of the strain tensor $\underset{\sim}{\varepsilon}$ and the strain-gradient tensor $\underset{\sim}{\sim}$. This coupled constitutive law requires tensors belonging to the following spaces:

$$
\underset{\approx}{\mathrm{C}} \in \mathbb{E} \mathrm{la}_{4} \simeq \mathcal{L}^{s}\left(\mathbb{T}_{(i j)}, \mathbb{T}_{(i j)}\right), \quad \underset{\approx}{\mathrm{M}} \in \mathbb{E} \mathrm{la}_{5} \simeq \mathcal{L}\left(\mathbb{T}_{(i j) k}, \mathbb{T}_{(i j)}\right), \quad \underset{\approx}{\mathrm{A}} \in \mathbb{E} \mathrm{la}_{6} \simeq \mathcal{L}^{s}\left(\mathbb{T}_{(i j) k}, \mathbb{T}_{(i j) k}\right)
$$

In this model we have:

- State tensors: $\underset{\sim}{\sigma}, \underset{\sim}{\varepsilon}, \underset{\sim}{\tau} \underset{\sim}{\eta}$

- Constitutive tensors: $\underset{\approx}{\mathrm{C}} \underset{\approx}{\mathrm{M}}$ and $\underset{\approx}{\mathrm{A}}$.

To be more specific, the constitutive equations read:

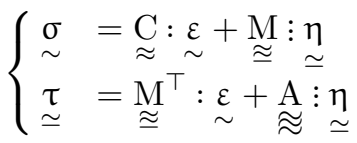

where

- $\underset{\approx}{\mathrm{C}} \in \mathbb{E} \mathrm{Ela}_{4}:=\left\{\underset{\approx}{\mathrm{T}} \in \stackrel{4}{\otimes} \mathbb{R}^{2} \mid \underset{\approx}{\mathrm{T}} \in \mathbb{T}_{\underline{(i j)}} \underline{(k l)}\right\}$ is the fourth-order elasticity tensor;

- $\underset{\approx}{\mathrm{M}} \in \mathbb{E} \mathrm{la}_{5}:=\left\{\underset{\approx}{\mathrm{T}} \in \stackrel{5}{\otimes} \mathbb{R}^{2} \mid \underset{\approx}{\mathrm{T}} \in \mathbb{T}_{(i j)(k l) m}\right\}$ is the fifth-order elasticity tensor;

- $\underset{\approx}{\mathrm{M}^{\top}} \in \mathbb{E}^{\top} \mathrm{a}_{5}^{\top}:=\left\{\underset{\approx}{\mathrm{T}} \in \stackrel{5}{\otimes} \mathbb{R}^{2} \mid \underset{\approx}{\mathrm{T}} \in \mathbb{T}_{(i j) k(l m)}\right\}$ is the fifth-order elasticity tensor defined as the transpose of $\underset{\approx}{\mathrm{M}}$ in the following sense $\left(\underset{\widetilde{\mathrm{M}}}{\mathrm{M}^{\top}}\right)_{i j k l m}=M_{l m i j k}$;

- $\underset{\approx}{\mathrm{A}} \in \mathbb{E} \operatorname{Ela}_{6}:=\left\{\underset{\mathrm{T}}{\mathrm{T}} \in \stackrel{6}{\approx} \mathbb{R}^{2} \mid \underset{\approx}{\mathrm{T}} \in \mathbb{T}_{\underline{(i j) k}} \underline{(l m) n}\right\}$ is the sixth-order elasticity tensor.

Let's define $\mathcal{S}$ grd the tensor space of the strain-gradient constitutive tensors as

$$
\mathcal{S g r d}=\mathbb{E l a}{ }_{4} \oplus \mathbb{E} \mathrm{la}_{5} \oplus \mathbb{E} \mathrm{la}_{6} .
$$

A strain-gradient elastic law is defined by a triplet $\mathcal{E}:=(\underset{\approx}{\mathrm{C}} \underset{\approx}{\mathrm{M}}, \underset{\approx}{\mathrm{A}}) \in \mathcal{S}$ grd .

2.2. Harmonic structure of constitutive tensors. When a material is rotated ${ }^{4}$ its physical nature is not affected but, with respect to a fixed reference, its constitutive tensors are transformed. Since constitutive tensors are usually of order greater than 2, the way they transform is not simple and their different parts transform differently: some components are left fixed while others turn at different speeds. The different mechanisms of transformation of a tensor with respect to an orthogonal transformation are revealed by its harmonic structure ${ }^{5}$. The harmonic decomposition consists in decomposing a finite-dimensional vector space into a direct sum of $\mathrm{O}(2)$-irreducible subspaces. A subspace $\mathbb{K}$ of $\mathbb{T}^{n}$ is called $\mathrm{O}(2)$-irreducible if: 1) it is $\mathrm{O}(2)$-invariant (i.e., $\mathbf{g} \star \mathrm{T} \in \mathbb{K}$ for all $\mathbf{g} \in \mathrm{O}(2)$ and $\mathrm{T} \in \mathbb{K}) ; 2$ ) its only invariant subspaces are itself and the null space. It is known that $\mathrm{O}(2)$-reducible spaces are isomorphic to a direct sum of harmonic tensor spaces

\footnotetext{
${ }^{4}$ Here rotated is understood in the broad sense of a full orthogonal transformation.

${ }^{5}$ The explicit harmonic decomposition is just an explicit expression of this harmonic structure.
} 
$\mathbb{K}^{n}[38,9]$. Such a decomposition is interesting since the $\mathrm{O}(2)$-action on $\mathbb{K}^{n}$ is elementary and given by $\rho_{n}$ [9], with for $n \geq 1$ :

$$
\rho_{n}(\mathbf{r}(\theta)):=\left(\begin{array}{cc}
\cos n \theta & -\sin n \theta \\
\sin n \theta & \cos n \theta
\end{array}\right), \quad \rho_{n}\left(\boldsymbol{\pi}\left(\underline{\mathrm{e}}_{2}\right)\right):=\left(\begin{array}{cc}
1 & 0 \\
0 & -1
\end{array}\right) .
$$

The $\mathrm{O}(2)$-action on $\mathbb{K}^{0}$ is the identity and the $\mathrm{O}(2)$-action on $\mathbb{K}^{-1}$ is given by the determinant of the transformation:

$$
\rho_{0}(\mathbf{Q}):=1, \quad \rho_{-1}(\mathbf{Q}):=\operatorname{det} \mathbf{Q} .
$$

The harmonic structure of a tensor space can be determined without making heavy computations by using the Clebsch-Gordan formula. This formula indicates how the tensor product of two irreducible spaces decomposes into a direct sum of irreducible spaces. Note that this formula only indicates the structure of the resulting vector space and does not provide an explicit construction of the decomposition. The construction of an associated explicit decomposition will be undertaken in Sections 4 and 5. For the determination of the harmonic structure, we use the following result, the proof of which is found in [9].

Lemma 2.1. For every integers $p>0$ and $q>0$, we have the following isotypic decompositions, where the meaningless products are indicated by $\times$ :

\begin{tabular}{|c|c|c|c|}
\hline$\otimes$ & $\mathbb{K}^{q}$ & $\mathbb{K}^{0}$ & $\mathbb{K}^{-1}$ \\
\hline $\mathbb{K}^{p}$ & $\left\{\begin{array}{cc}\mathbb{K}^{p+q} \oplus \mathbb{K}^{|p-q|}, & p \neq q \\
\mathbb{K}^{2 p} \oplus \mathbb{K}^{0} \oplus \mathbb{K}^{-1}, & p=q\end{array}\right.$ & $\mathbb{K}^{p}$ & $\mathbb{K}^{p}$ \\
\hline $\mathbb{K}^{0}$ & $\mathbb{K}^{q}$ & $\mathbb{K}^{0}$ & $\mathbb{K}^{-1}$ \\
\hline $\mathbb{K}^{-1}$ & $\mathbb{K}^{q}$ & $\mathbb{K}^{-1}$ & $\mathbb{K}^{0}$ \\
\hline
\end{tabular}

\begin{tabular}{|c|c|c|c|}
\hline$\otimes^{s}$ & $\mathbb{K}^{p}$ & $\mathbb{K}^{0}$ & $\mathbb{K}^{-1}$ \\
\hline $\mathbb{K}^{p}$ & $\mathbb{K}^{2 p} \oplus \mathbb{K}^{0}$ & $\times$ & $\times$ \\
\hline $\mathbb{K}^{0}$ & $\times$ & $\mathbb{K}^{0}$ & $\times$ \\
\hline $\mathbb{K}^{-1}$ & $\times$ & $\times$ & $\mathbb{K}^{0}$ \\
\hline
\end{tabular}

Using the previous result, we can determine the harmonic structure of the state tensor spaces and constitutive tensor spaces of the strain-gradient elasticity:

\begin{tabular}{|c||c|}
\hline State tensor space & Harmonic structure \\
\hline \hline $\mathbb{T}_{(i j)}$ & $\mathbb{K}^{2} \oplus \mathbb{K}^{0}$ \\
\hline $\mathbb{T}_{(i j) k}$ & $\mathbb{K}^{3} \oplus 2 \mathbb{K}^{1}$ \\
\hline
\end{tabular}

\begin{tabular}{|c||c|}
\hline Constitutive tensor space & Harmonic structure \\
\hline \hline $\mathbb{E l a}_{4}$ & $\mathbb{K}^{4} \oplus \mathbb{K}^{2} \oplus 2 \mathbb{K}^{0}$ \\
\hline $\mathbb{E} \mathrm{la}_{5}$ & $\mathbb{K}^{5} \oplus 3 \mathbb{K}^{3} \oplus 5 \mathbb{K}^{1}$ \\
\hline $\mathbb{E} \mathrm{la}_{6}$ & $\mathbb{K}^{6} \oplus 2 \mathbb{K}^{4} \oplus 5 \mathbb{K}^{2} \oplus 4 \mathbb{K}^{0} \oplus \mathbb{K}^{-1}$ \\
\hline
\end{tabular}

TABLE 1. Irreducible decompositions of state tensor spaces (left table) and constitutive tensor spaces (right table).

The challenge of future sections will be to explicitly construct the harmonic decompositions corresponding to these structures. It should be pointed out that as soon as the harmonic structure involves multiple spaces of the same order, the explicit decomposition is not uniquely defined [38]. As can be seen from the previous tables, this is the case for all the constitutive tensor spaces considered in this study.

\section{HARMONiC DECOMPOSition: METHOdology}

In this section, we present the geometric objects and methods that are at the core of our approach to decompose tensors. With the exception of Proposition 3.2 which must be adapted, the results provided in this section are valid for 2D and 3D physical spaces. The 3D situation will be detailed in a future contribution, and we will focus here only on the $2 \mathrm{D}$ case. 
3.1. The harmonic decomposition. Let $\mathbb{V}$ and $\mathbb{W}$ be two vector spaces, a map $\phi: \mathbb{V} \rightarrow \mathbb{W}$ is said to be $\mathrm{O}(2)$-equivariant if

$$
\forall \mathbf{g} \in \mathrm{O}(2), \forall \mathbf{v} \in \mathbb{V}, \mathbf{g} \star \phi(\mathbf{v})=\phi(\mathbf{g} \star \mathbf{v})
$$

An explicit harmonic decomposition $\phi$ of a tensor $\mathbf{T} \in \mathbb{T}^{n}$ is an $\mathrm{O}(2)$-equivariant linear isomorphism between a direct sum of harmonic spaces $\mathbb{V} \simeq \bigoplus \mathbb{K}^{k_{i}}$ and the space $\mathbb{T}^{n}$ :

$$
\begin{aligned}
\phi: \mathbb{V} \simeq \bigoplus \mathbb{K}^{k_{i}} & \rightarrow \mathbb{T}^{n} \\
\left(\alpha, \ldots, \mathbf{K}^{n}\right) & \mapsto \mathbf{T}=\phi\left(\alpha, \ldots, \mathbf{K}^{n}\right)
\end{aligned}
$$

Since this isomorphism is $\mathrm{O}(2)$-equivariant [9], it satisfies the following property:

$$
\forall \mathbf{g} \in \mathrm{O}(2), \mathbf{g} \star \mathbf{T}=\mathbf{g} \star \phi\left(\alpha, \ldots, \mathbf{K}^{n}\right)=\phi\left(\alpha, \ldots, \mathbf{g} \star \mathbf{K}^{n}\right)
$$

which means that rotating $\mathbf{T}$ is equivalent to rotating the elements of its decomposition, and vice versa. Since the transformations of the harmonic components (i.e. the elements of the harmonic decomposition) are elementary (cf. Equations (2.3)), it is generally easier to study the transformations of the harmonic components rather than the ones of the full tensor. In this view, Equation (3.1) provides an explicit link between these two representations. The challenge is to obtain such an explicit expression for $\phi$.

3.2. A three-step methodology. Consider two spaces of state tensors denoted by $\mathbb{E}$ and $\mathbb{F}$. The (linear) constitutive law is an element $\mathbf{T} \in \mathcal{L}(\mathbb{E}, \mathbb{F})$. In the present context, $\mathbf{T}$ represents the constitutive tensor of which we want to obtain the harmonic decomposition. The construction of a Clebsch-Gordan Harmonic Decomposition (in abbreviated form CGHD) of $\mathbf{T}$ is obtained using the following procedure:

1) State Tensor Harmonic Decomposition (STHD): Choose and compute a harmonic decomposition for elements $\mathbf{v} \in \mathbb{E}$ and $\mathbf{w} \in \mathbb{F}$. This decomposition implies the definition of harmonic embedding operators. From these operators, we get a family of orthogonal projectors that will be used to decompose $\mathbf{T}$

2) Intermediate Block Decomposition (IBD): Consider an element $\mathbf{T} \in \mathcal{L}(\mathbb{E}, \mathbb{F})$ which represents the constitutive tensor of which we want to obtain the harmonic decomposition. The choice of a STHD and the use of the associated projectors induce a decomposition of $\mathcal{L}(\mathbb{E}, \mathbb{F})$ into "blocks". This decomposition, that will be referred to as the Intermediate Block Decomposition, is not irreducible;

3) Clebsch-Gordan Harmonic Decomposition (CGHD): Each elementary block of the Intermediate Block Decomposition belongs to a space $\mathbb{K}^{p} \otimes \mathbb{K}^{q}$, the harmonic structure of which is known by the Clebsch-Gordan formula. The use of harmonic embeddings allows us to break down each block into irreducible tensors.

The combination of the last two steps provides the Clebsch-Gordan Harmonic Decomposition of $\mathbf{T} \in$ $\mathcal{L}(\mathbb{E}, \mathbb{F})$. The resulting decomposition is a particular explicit harmonic decomposition of $\mathbf{T}$ which is compatible with the harmonic decompositions of $\mathbf{v}$ and $\mathbf{w}$. This decomposition is uniquely defined ${ }^{6}$ by the choice of a particular form of the harmonic decompositions for the spaces $\mathbb{E}$ and $\mathbb{F}$. It has to be noted that different choices for the decompositions of $\mathbb{E}$ and $\mathbb{F}$ will lead to different decompositions of $\mathbf{T}$.

3.3. Harmonic embeddings. The isotypic decomposition of a tensor space $\mathbb{T}^{n}$ can be written in two different, but isomorphic, ways:

$$
\mathbb{T}^{n} \simeq \bigoplus_{k=-1}^{n} \bigoplus_{l=0}^{p_{k}} \mathbb{H}_{l}^{(n, k)} \simeq \bigoplus_{k=-1}^{n} \bigoplus_{l=0}^{p_{k}} \mathbb{K}_{l}^{k}
$$

in which the involved spaces are:

$\mathbb{T}^{n}$ : a space of $n$ th-order tensors with given index symmetries, it is the tensor space we want to decompose;

$\mathbb{H}^{(n, k)}$ : a subspace of $\mathbb{T}^{n}$ isomorphic to $\mathbb{K}^{k}$, it is the embedding space for the elements belonging to $\mathbb{K}^{k}$;

$\mathbb{K}^{k}$ : a space of $k$ th-order harmonic tensors, the elements of this space are used to parametrize the harmonic decomposition of $\mathbf{T} \in \mathbb{T}^{n}$.

\footnotetext{
${ }^{6}$ This claimed uniqueness is ensured by the property that in $2 \mathrm{D}$ the tensor product of irreducible harmonic spaces decomposes, as detailed in the tables of Lemma 2.1, into a direct sum of irreducible spaces of distinct orders. Even if the Clesbch-Gordan formula are different, this property is also valid in 3D [45].
} 
In the first decomposition of Equation (3.2), $\mathbb{H}^{(n, k)}$ is a subspace of $\mathbb{T}^{n}$ isomorphic to $\mathbb{K}^{k}$ with $k \leq n$, while in the second one $\mathbb{K}^{k}$ is a subspace of $\mathbb{G}^{k}$. In both decompositions, the first direct sum is on the order of the harmonic space, while the other one concerns the summation of the different spaces of the same order. The space $\mathbb{H}^{(n, k)}$ serves as an intermediate space to embed a tensor $\mathbf{K} \in \mathbb{K}^{k}$ into $\mathbf{T} \in \mathbb{T}^{n}$. Thus, the elements of $\mathbb{H}^{(n, k)}$ are parametrized by elements from $\mathbb{K}^{k}$, this parametrization is what we call the harmonic embedding. This technique will be used repeatedly in our work. As will be seen in Section 4, this allows us to express the harmonic decompositions in terms of projection operators.

Let us consider more precisely the parametrization of $\mathbb{H}^{(n, k)}$ by $\mathbb{K}^{k}$. The connections of the different spaces are shown on the following diagram:

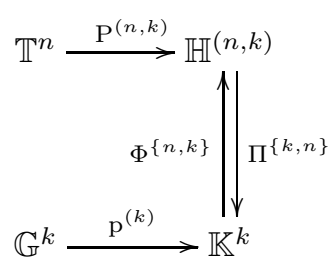

In this diagram, the associated mappings are:

$\mathrm{P}^{(n, k)}$ : a projector from $\mathbb{T}^{n}$ to its subspace $\mathbb{H}^{(n, k)}, \mathrm{P}^{(n, k)}$ is a $2 n$ th-order tensor;

$\mathrm{p}^{(k)}$ : a projector from $\mathbb{G}^{k}$ to its subspace $\mathbb{K}^{k}, \mathrm{p}^{(k)}$ is a $2 k$ th-order tensor;

$\Pi^{\{k, n\}}$ : a projector from $\mathbb{T}^{n}$ to its subspace $\mathbb{K}^{k}, \Pi^{\{k, n\}}$ is a $(n+k)$ th-order tensor ${ }^{7}$;

$\Phi^{\{n, k\}}$ : a harmonic embedding of $\mathbb{K}^{k}$ into $\mathbb{T}^{n}, \Phi^{\{n, k\}}$ is a $(n+k)$ th-order tensor.

By definition, we have the following fundamental relations:

$$
\mathrm{I}^{\mathbb{H}^{(n, k)}}:=\Phi^{\{n, k\}} \stackrel{(k)}{\cdot} \Pi^{\{k, n\}} \in \mathbb{I}^{2 n}, \quad \mathbb{I}^{\mathbb{K}^{k}}:=\Pi^{\{k, n\}} \stackrel{(n)}{\cdot} \Phi^{\{n, k\}} \in \mathbb{I}^{2 k}
$$

and, by construction,

$$
\mathrm{P}^{(n, k)}=\mathrm{I}^{\mathbb{H}^{(n, k)}}, \quad \mathrm{p}^{(k)}=\mathrm{I}^{\mathbb{K}^{k}} .
$$

We have the remarkable property that all these different operators are known as soon as $\Phi^{\{n, k\}}$ is determined. To see that, let us first define $\Phi^{\{k, n\}}:=\left(\Phi^{\{n, k\}}\right)^{T}$ to be the transpose of $\Phi^{\{n, k\}}$, i.e. the tensor which satisfies the following property:

$$
\forall \mathbf{V} \in \mathbb{T}^{n}, \forall \mathbf{v} \in \mathbb{K}^{k}, \quad\left\langle\mathbf{V}, \Phi^{\{n, k\}} \stackrel{(k)}{\cdot} \mathbf{v}\right\rangle_{\mathbb{T}^{n}}=\left\langle\Phi^{\{k, n\}} \stackrel{(n)}{\cdot} \mathbf{V}, \mathbf{v}\right\rangle_{\mathbb{K}^{k}} .
$$

In terms of components, we have

$$
\left(\Phi^{\{k, n\}}\right)_{i_{1} \ldots i_{n+k}}=\left(\Phi^{\{n, k\}}\right)_{i_{n+1} \ldots i_{n+k} i_{1} \ldots i_{n}} .
$$

We have the following theorem:

Theorem 3.1. Let $\Phi^{\{n, k\}}$ be a harmonic embedding of $\mathbb{K}^{k}$ into $\mathbb{T}^{n}$. The operators $\mathrm{P}^{(n, k)}, \mathrm{p}^{(k)}, \Pi^{\{k, n\}}$ defined above can be expressed in terms of $\Phi^{\{n, k\}}$ as follows:

$$
\Pi^{\{k, n\}}=\frac{1}{\gamma} \Phi^{\{k, n\}}, \quad \mathrm{P}^{(n, k)}=\frac{1}{\gamma} \Phi^{\{n, k\}} \stackrel{(k)}{\cdot} \Phi^{\{k, n\}}, \quad \mathrm{p}^{(k)}=\frac{1}{\gamma} \Phi^{\{k, n\}} \stackrel{(n)}{\cdot} \Phi^{\{n, k\}}
$$

in which $\Phi^{\{k, n\}}$ denotes the transpose of $\Phi^{\{n, k\}}, \gamma$ is defined as

$$
\gamma=\frac{\| \Phi^{\{n, k\}} \cdot(k)}{\|\mathbf{v}\|^{2}}, \mathbf{v} \in \mathbb{K}^{k} \backslash\{0\}
$$

and we adopt the convention that, in the case $k=0, \stackrel{(0)}{\cdot}=\otimes$.

Proof. The proof is made by inserting the result of Lemma A.5 into the relations provided by combining Equations (3.4) and (3.5). Intermediate lemmas are provided in Appendix A.

\footnotetext{
${ }^{7}$ In the notation $\Pi^{\{k, n\}}$ the order of the bracketed exponents is organized so that the left-most exponent indicates the tensor order of the image of the map, while the right-most exponent is the tensor order of the argument. This convention does not apply for in-parenthesis exponents such as those appearing in $\mathrm{P}^{(n, k)}$ which is a $2 n$ th-order tensor and not a $(n+k)$ th-order one.
} 
The following proposition, which is demonstrated in Appendix A, gives another formula for the calculation of the constant $\gamma$ :

Proposition 3.2. The constant $\gamma$ in Theorem 3.1 can be calculated as

$$
\gamma=\frac{1}{2} \operatorname{tr} \mathrm{M}
$$

in which $\mathrm{M}$ is the matrix of the linear map $\eta: \mathbb{K}^{k} \rightarrow \mathbb{K}^{k}$ defined by

$$
\eta(\mathbf{v})=\left(\Phi^{\{k, n\}} \circ \Phi^{\{n, k\}}\right) \cdot \mathbf{v} .
$$

\section{Application to classical elasticity}

The aim of the present section is to detail the method to the well-known situation of the fourth-order elasticity tensor. Results obtained here can be checked with the results available in the literature [76, 26]. The presentation is here mainly illustrative and will be extended in the next section to the more complicated situation of strain-gradient elasticity for which results are original and not available in the literature. Let us begin by the construction of the harmonic decomposition of the state tensor space $\mathbb{T}_{(i j)}$.

4.1. Step 1: Decomposition of the state tensor space $\mathbb{T}_{(i j)}$. As indicated in Table $2.2, \mathbb{T}_{(i j)}$ has the following harmonic structure:

$$
\mathbb{T}_{(i j)} \simeq \mathbb{H}^{(2,2)} \oplus \mathbb{H}^{(2,0)} \simeq \mathbb{K}^{2} \oplus \mathbb{K}^{0}
$$

The decomposition of an element $\underset{\sim}{\mathrm{t}} \in \mathbb{T}_{(i j)}$ is uniquely defined and it corresponds to the partition of $\mathrm{t}_{\sim}$ into a deviatoric tensor $\underset{\sim}{\mathrm{d}} \in \mathbb{H}^{(2,2)}=\widetilde{\mathbb{K}}^{2}$ and a spherical one $\underset{\sim}{\mathrm{s}} \in \mathbb{H}^{(2,0)}$. The identity tensor on $\mathbb{T}_{(i j)}$ can be decomposed as the sum of the deviatoric and spherical projectors denoted $\underset{\approx}{\mathrm{P}}(2,2)$ and $\underset{\approx}{\mathrm{P}}(2,0)$ :

$$
\mathrm{I}^{\mathrm{T}_{(i j)}}=\underset{\approx}{\mathrm{P}}(2,2)+\underset{\approx}{\mathrm{P}^{(2,0)}} \text {. }
$$

The structure of the associated harmonic embeddings are described on the following diagrams

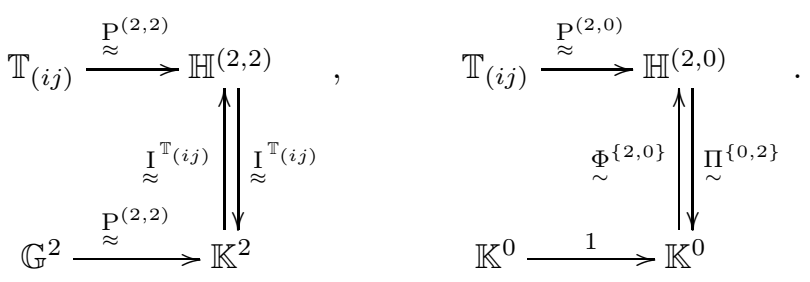

Let us build the spherical projector $\underset{\approx}{\mathrm{P}^{(2,0)}}$ first, the deviatoric projector $\underset{\approx}{\mathrm{P}^{(2,2)}}$ will then be deduced from it. Following the harmonic embedding method, the spherical part of $t$ will be parametrized by a scalar $\alpha \in \mathbb{K}^{0}$. To construct the associated projector, let us first determine the embedding operator ${\underset{\sim}{\sim}}^{\{2,0\}}$ :

$$
\Phi_{\sim}^{\{2,0\}}=\lambda \underset{\sim}{\mathrm{i}^{(2)}}
$$

where $\lambda \in \mathbb{R} \backslash\{0\}$ is a free scaling factor. In this situation, we have $\underset{\sim}{\Phi^{\{2,0\}}}={\underset{\sim}{\Phi}}^{\{0,2\}}$. From Theorem 3.1, $\Pi_{\sim}^{\{0,2\}}$ has the following expression:

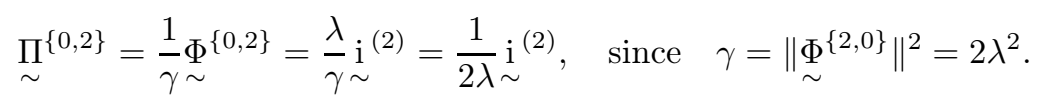

This results in the following expression for $\underset{\approx}{\mathrm{P}}{ }^{(2,0)}$ :

$$
\underset{\approx}{\mathrm{P}^{(2,0)}}=\underset{\sim}{\Pi^{\{2,0\}}} \otimes \underset{\sim}{\Phi^{\{0,2\}}}=2 \underset{\sim}{\Phi^{\{2,0\}}} \otimes \underset{\sim}{\Phi^{\{0,2\}}}=\frac{1}{2} \mathrm{i}_{\sim}^{(2)} \otimes \underset{\sim}{\mathrm{i}^{(2)}}=\frac{1}{2} \mathrm{i}_{\approx}^{(4)} .
$$


We recognise here the well-known expression of the spherical projector. Note that the choice of a specific $\lambda$ has no consequence on the expression of $\underset{\approx}{\mathrm{P}}(2,0)$. The deviatoric projector can then be obtained:

$$
\underset{\approx}{\mathrm{P}^{(2,2)}}:={\underset{\approx}{\mathrm{I}}}^{\mathrm{T}_{(i j)}}-\underset{\approx}{\mathrm{P}^{(2,0)}}=\frac{1}{2}\left({\underset{\approx}{\mathrm{i}}}_{2}^{(4)}+{\underset{\approx}{\mathrm{i}}(4)}_{\mathrm{i}_{3}}^{\mathrm{i}_{\approx}^{(4)}}\right)
$$

Remark 4.1. From Lemma A.6, it appears that the tensors $\underset{\approx}{\mathrm{P}^{(2,2)}}$ and $\underset{\approx}{\mathrm{P}^{(2,0)}}$ can be considered as isotropic elasticity tensors in $\mathbb{E l a}_{4}$. Interpreted as elements of $\left(\mathbb{E l a}_{4},::\right)$, these tensors are associated to the following Gram matrix:

\begin{tabular}{|c|c|c|}
\hline :: & $\mathrm{P}^{(2,2)}$ & $\mathrm{P}^{(2,0)}$ \\
\hline $\mathrm{P}^{(2,2)}$ & 2 & 0 \\
\hline $\mathrm{P}^{(2,0)}$ & 0 & 1 \\
\hline
\end{tabular}

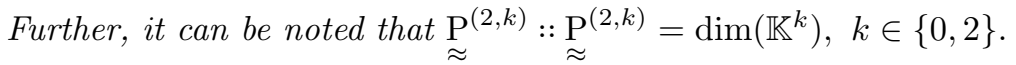

Although the value of $\lambda$ has no importance for the expression of the projectors, it has some to construct an explicit parametrization of $\underset{\sim}{\mathrm{t}}$ in terms of its harmonic components $\underset{\sim}{\mathrm{d}}$ and $\alpha$. For our concern, the value of $\lambda$ will be chosen by imposing $\underset{\sim}{\sim} \prod^{\{0,2\}}$ to be the standard trace operator, i.e.

$$
\prod_{\sim}^{\{0,2\}}: \underset{\sim}{\mathrm{t}}=\frac{1}{2 \lambda} \underset{\sim}{\mathrm{i}}(2): \underset{\sim}{\mathrm{t}}=\operatorname{tr}(\underset{\sim}{\mathrm{t}})
$$

which sets $\lambda$ to $\frac{1}{2}$. Collecting all the previous observations we obtain the following results:

Proposition 4.2. There exists an $\mathrm{O}(2)$-equivariant isomorphism $\varphi$ between $\mathbb{T}_{(i j)}$ and $\mathbb{K}^{2} \oplus \mathbb{K}^{0}$ such that

$$
\underset{\sim}{\mathrm{t}}=\underset{\sim}{\mathrm{d}}+\underset{\sim}{\mathrm{s}}=\underset{\sim}{\mathrm{d}}+\underset{\sim}{\Phi^{\{2,0\}}} \alpha=\varphi(\underset{\sim}{\mathrm{d}}, \alpha)
$$

with $(\underset{\sim}{\mathrm{d}}, \alpha) \in \mathbb{K}^{2} \times \mathbb{K}^{0}$ and $\underset{\sim}{\Phi^{\{2,0\}}}$ is such that

$$
\underset{\sim}{\mathrm{s}}={\underset{\sim}{\Phi}}^{\{2,0\}} \alpha \quad \text { with } \quad{\underset{\sim}{\Phi}}^{\{2,0\}}:=\frac{1}{2} \mathrm{i}_{\sim}^{(2)} .
$$

Conversely, we have

$$
\left.\alpha=\prod_{\sim} \prod^{\{0,2\}}: \underset{\sim}{\mathrm{t}}=\operatorname{tr} \underset{\sim}{\mathrm{t}}\right) \text { with } \underset{\sim}{\Pi^{\{0,2\}}}={\underset{\sim}{\mathrm{i}}}^{(2)} .
$$

4.2. Step 2: Intermediate Block Decomposition of $\mathbb{E}^{2} a_{4}$. In this subsection, and in the following ones, results will be provided under two forms:

(1) the general one which involves embedding operators (as will be used in the next section);

(2) the simplified one, since for the particular case of the elasticity tensor the operators have very simple expressions.

Using the family of projectors $\left.\underset{\approx}{\left(\mathrm{P}^{(2,2)}\right.}, \underset{\approx}{\mathrm{P}^{(2,0)}}\right)$ constructed from the harmonic decomposition of $\mathbb{T}_{(i j)}$, we can demonstrate the following result:

Proposition 4.3. The tensor $\underset{\approx}{\mathrm{C}} \in \mathbb{E} \mathrm{la}_{4}$ admits the uniquely defined Intermediate Block Decomposition associated to the family of projectors $\underset{\approx}{\mathrm{P}}(2,2), \underset{\approx}{\mathrm{P}(2,0)})$ :

$$
\begin{aligned}
& \underset{\approx}{\mathrm{C}}=\underset{\approx}{\mathrm{C}^{2,2}}+2\left(\underset{\sim}{\mathrm{h}^{2,0}} \otimes \underset{\sim}{\Phi^{\{0,2\}}}+\underset{\sim}{\Phi^{\{2,0\}}} \otimes \underset{\sim}{\mathrm{h}^{2,0}}\right)+2 \alpha^{0,0}{\underset{\sim}{\mathrm{P}}}^{(2,0)}
\end{aligned}
$$

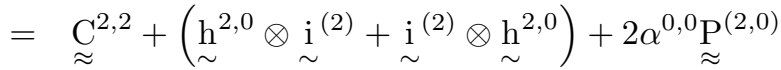


in which $\left.\underset{\approx}{\mathrm{C}^{2,2}}, \mathrm{~h}^{2,0}, \alpha^{0,0}\right)$ are elements of $\left(\mathbb{K}^{2} \otimes^{s} \mathbb{K}^{2}\right) \times \mathbb{K}^{2} \times \mathbb{K}^{0}$. Those elements are defined from $\underset{\approx}{\mathrm{C}}$ as follows:

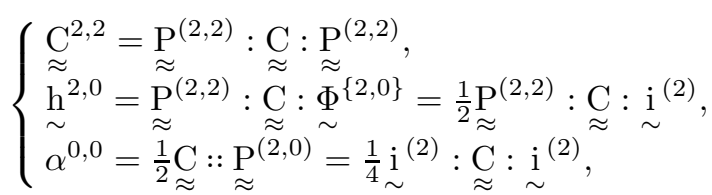

where $\Phi^{\{2,0\}}$ is defined in Proposition 4.2.

Proof. Let consider $\underset{\sim}{\mathrm{t}}$ and $\underset{\sim}{\mathrm{t}^{\star}}$ in $\mathbb{T}_{(i j)}$ such as $\underset{\sim}{\mathrm{t}^{\star}}=\underset{\sim}{\mathrm{C}}: \underset{\sim}{\mathrm{t}}$. The elements $\underset{\sim}{\mathrm{t}}$ and $\underset{\sim}{\mathrm{t}^{\star}}$ can be decomposed as

$$
\underset{\sim}{\mathrm{t}}=\underset{\sim}{\mathrm{d}}+\underset{\sim}{\mathrm{s}}, \underset{\sim}{\mathrm{t}^{\star}}=\mathrm{d}_{\sim}^{\star}+\underset{\sim}{\mathrm{s}^{\star}}
$$

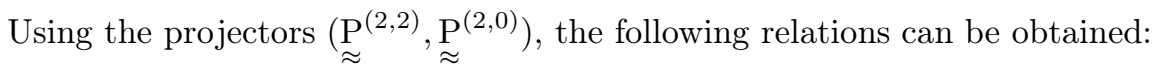

$$
\stackrel{\mathrm{t}}{\star}^{\star}=\underset{\approx}{\mathrm{P}^{(2,2)}}:{\underset{\sim}{\mathrm{t}}}^{\star}+\underset{\approx}{\mathrm{P}^{(2,0)}}: \underset{\sim}{\mathrm{t}^{\star}} \text { and } \underset{\sim}{\mathrm{t}^{\star}}=\left(\underset{\approx}{\mathrm{C}}: \underset{\approx}{\mathrm{P}^{(2,2)}}\right): \underset{\sim}{\mathrm{t}}+\left(\underset{\approx}{\mathrm{C}}: \underset{\approx}{\mathrm{P}^{(2,0)}}\right): \underset{\sim}{\mathrm{t}} .
$$

Through their combination the constitutive law $\underset{\sim}{\mathrm{t}^{\star}}=\underset{\approx}{\mathrm{C}}: \underset{\sim}{\mathrm{t}}$ can be expressed as

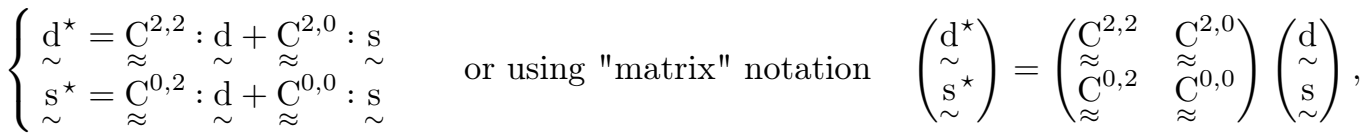

in which:

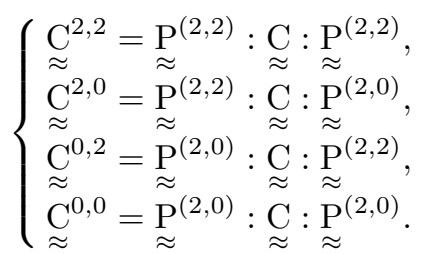

Expressed under this form, the symmetry of the constitutive law is not obvious. Using the relation

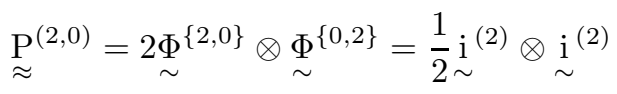

the previous relationships can be rewritten as follows

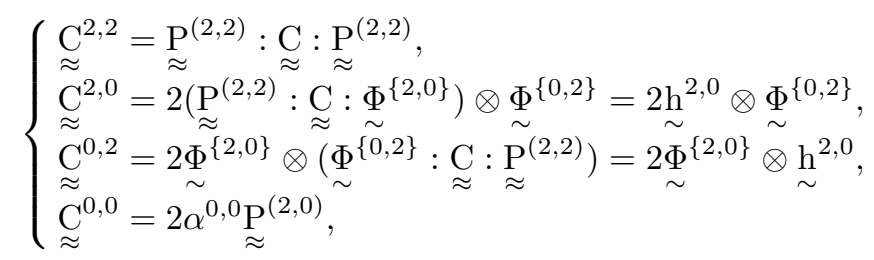

where the following intermediate quantities have been introduced:

$$
{\underset{\sim}{\mathrm{h}}}^{2,0}=\underset{\approx}{\mathrm{P}}{ }^{(2,2)}: \underset{\approx}{\mathrm{C}}: \underset{\sim}{\Phi^{\{2,0\}}}, \quad \alpha^{0,0}=\underset{\sim}{\Phi^{\{0,2\}}}: \underset{\approx}{\mathrm{C}}: \underset{\sim}{\Phi^{\{2,0\}}}=\frac{1}{2} \underset{\approx}{\mathrm{P}^{(2,0)}}:: \underset{\approx}{\mathrm{C}} .
$$

Therefore, we obtain:

$$
\begin{aligned}
& \underset{\mathrm{t}^{\star}}{\sim} \underset{\sim}{\mathrm{d}^{\star}}+\underset{\sim}{\mathrm{s}^{\star}} \\
& =\left(\underset{\sim}{\mathrm{C}^{2,2}}+2 \underset{\sim}{\mathrm{h}^{2,0}} \otimes \underset{\sim}{\Phi^{\{0,2\}}}\right): \underset{\sim}{\mathrm{d}}+\left(2 \underset{\sim}{\Phi^{\{2,0\}}} \otimes \underset{\sim}{\mathrm{h}^{2,0}}+2 \alpha^{0,0} \mathrm{P}^{(2,0)}\right): \underset{\sim}{\mathrm{s}}
\end{aligned}
$$

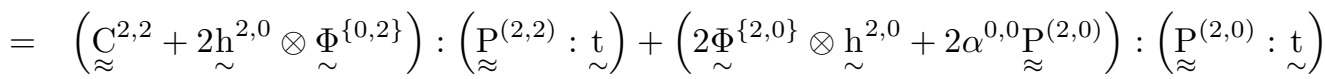

$$
\begin{aligned}
& =\left(\underset{\approx}{\mathrm{C}^{2,2}}+2\left(\underset{\sim}{\Phi^{\{2,0\}}} \otimes \underset{\sim}{\mathrm{h}^{2,0}}+\underset{\sim}{\mathrm{h}^{2,0}} \otimes \underset{\sim}{\Phi^{\{0,2\}}}\right)+2 \alpha^{0,0} \mathrm{P}^{(2,0)}\right): \underset{\sim}{\mathrm{t}}
\end{aligned}
$$

and hence, by identification:

$$
\underset{\approx}{\mathrm{C}}=\underset{\approx}{\mathrm{C}^{2,2}}+2\left(\underset{\sim}{\Phi^{\{2,0\}}} \otimes \underset{\sim}{\mathrm{h}^{2,0}}+\underset{\sim}{\mathrm{h}^{2,0}} \otimes \underset{\sim}{\Phi^{\{0,2\}}}\right)+2 \alpha^{0,0} \mathrm{P}_{\approx}^{(2,0)} .
$$


4.3. Step 3: Clebsch-Gordan Harmonic Decomposition of $\mathbb{E}$ la $_{4}$. The Intermediate Block Decomposition given by Equation (4.6) for the elasticity tensor is not irreducible, meaning that some of its components can further be decomposed. To determine which components can be reduced and how to reduce them, the Clebsch-Gordan formula is essential. By construction, $\underset{\approx}{\mathrm{C}, 2} \in \mathbb{K}^{2} \otimes^{s} \mathbb{K}^{2}, \mathrm{\sim}^{2,0} \in \mathbb{K}^{2} \otimes \mathbb{K}^{0}$ and $\alpha^{0,0} \in \mathbb{K}^{0} \otimes^{s} \mathbb{K}^{0}$. By applying the Clebsch-Gordan formula (cf. Lemma 2.1) to each one of these spaces we obtain:

$$
\mathbb{K}^{2} \otimes \mathbb{K}^{0} \simeq \mathbb{K}^{2}, \quad \mathbb{K}^{0} \otimes^{s} \mathbb{K}^{0} \simeq \mathbb{K}^{0}, \quad \mathbb{K}^{2} \otimes^{s} \mathbb{K}^{2} \simeq \mathbb{K}^{4} \oplus \mathbb{K}^{0},
$$

which indicates that:

- the components $\left(\mathrm{h}^{2,0}, \alpha^{0,0}\right) \in \mathbb{K}^{2} \times \mathbb{K}^{0}$ are already irreducible;

- the component $\underset{\approx}{\mathrm{C}^{2,2}}$ is reducible and can be decomposed into a scalar and a fourth-order harmonic tensor.

To proceed the decomposition of $\underset{\approx}{\mathrm{C}^{2,2}}$ consider the following lemma which is a direct application of Theorem B.1:

Lemma 4.4. Tensors $\underset{\approx}{\mathrm{T}^{2,2}} \in \mathbb{K}^{2} \otimes^{s} \mathbb{K}^{2}$ admit the uniquely defined harmonic decomposition

$$
\underset{\approx}{\mathrm{T}^{2,2}}=\underset{\approx}{\mathrm{H}}+\frac{\alpha}{2} \mathrm{P}^{(2,2)}, \quad \text { with } \underset{\approx}{\mathrm{H}} \in \mathbb{K}^{4}, \alpha \in \mathbb{R}
$$

conversely

$$
\alpha=\underset{\approx}{\mathrm{T}^{2,2}}:: \underset{\approx}{\mathrm{P}}{ }^{(2,2)}, \quad \underset{\approx}{\mathrm{H}}=\underset{\approx}{\mathrm{T}^{2,2}}-\frac{\alpha}{2} \underset{\approx}{ } \mathrm{P}^{(2,2)} .
$$

Remark 4.5. The structure of the projections is summed up in the following diagram:

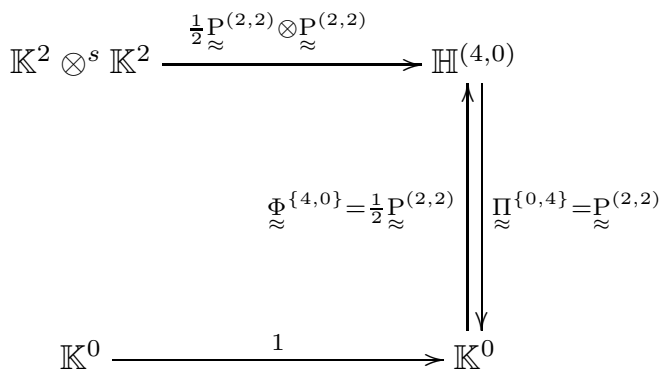

It can be observed that the method provides the intrinsic expression of the projector $\mathbf{P}^{(4,0)}$ from $\mathbb{K}^{2} \otimes^{s} \mathbb{K}^{2}$ onto $\mathbb{H}^{(4,0)}$ :

$$
\mathbf{P}^{(4,0)}=\frac{1}{2} \mathrm{P}^{(2,2)} \otimes \underset{\approx}{\mathrm{P}^{(2,2)}}
$$

The insertion of the result of Lemma 4.4 in the Intermediate Block Decomposition demonstrates the following proposition:

Proposition 4.6. The tensor $\underset{\approx}{\mathrm{C}} \in \mathbb{E} \mathrm{la}_{4}$ admits the uniquely defined Clebsch-Gordan Harmonic Decomposition associated to the family of projectors $\left.\underset{\approx}{\mathrm{P}}{ }^{(2,2)}, \underset{\approx}{\mathrm{P}}(2,0)\right)$ :

\begin{tabular}{|c|c|c|}
\hline $\mathbb{K}^{0}$ & $\mathbb{K}^{2}$ & $\mathbb{K}^{4}$ \\
\hline$\alpha^{0,0}=\underset{\approx}{\mathrm{P}^{(2,0)}}:: \underset{\approx}{\mathrm{C}}$ & $\stackrel{\mathrm{h}}{ }^{2,0}=\underset{\approx}{\mathrm{P}^{(2,2)}}: \underset{\approx}{\mathrm{C}}: \underset{\sim}{\Phi^{\{2,0\}}}$ & \\
\hline$\alpha^{2,2}={\underset{\approx}{(2,2}:: \mathrm{P}_{\approx}^{(2,2)}}^{\mathrm{P}^{2}}$ & & 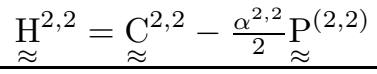 \\
\hline
\end{tabular}

$$
\underset{\approx}{\mathrm{C}}=\underset{\approx}{\mathrm{H}^{2,2}}+\frac{\alpha^{2,2}}{2} \underset{\approx}{\mathrm{P}^{(2,2)}}+2\left({\underset{\sim}{\mathrm{h}^{2,0}} \otimes \underset{\sim}{\Phi^{\{0,2\}}}+\underset{\sim}{\Phi}}^{\{2,0\}} \otimes \underset{\sim}{\mathrm{h}^{2,0}}\right)+2 \alpha^{0,0} \stackrel{\mathrm{P}}{(2,0)}^{(2,0)}
$$

in which $\left.\underset{\approx}{\mathrm{H}^{2,2}}, \mathrm{\sim}_{\sim}^{2,0}, \alpha^{2,2}, \alpha^{0,0}\right)$ are elements of $\mathbb{K}^{4} \times \mathbb{K}^{2} \times \mathbb{K}^{0} \times \mathbb{K}^{0}$ defined from $\underset{\approx}{\mathrm{C}}$ as follows:

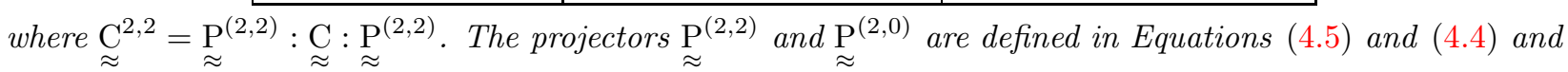
$\Phi^{\{2,0\}}$ is defined in Proposition 4.2. 
4.4. Link with symmetry classes. The symmetry classes ${ }^{8}$ of bidimensional elasticity tensor have been determined in $[41,16,76]$. The space $\mathbb{E} \mathrm{la}_{4}$ is divided into the following 4 symmetry classes:

$$
\Im\left(\mathbb{E} \mathrm{la}_{4}\right)=\left\{\left[\mathrm{Z}_{2}\right],\left[\mathrm{D}_{2}\right],\left[\mathrm{D}_{4}\right],[\mathrm{O}(2)]\right\},
$$

in which $\mathfrak{I}(\mathbb{T})$ is the set of symmetry classes of the space $\mathbb{T}$. These symmetry classes can be connected in the way indicated by the following diagram:

$$
\left[\mathrm{Z}_{2}\right] \longrightarrow\left[\mathrm{D}_{2}\right] \longrightarrow\left[\mathrm{D}_{4}\right] \longrightarrow[\mathrm{O}(2)]
$$

As discussed in [9], for a $\mathrm{Z}_{p}$-invariance, only the harmonic tensors of the order $k p, k \in \mathbb{N}$, are non-zero. The invariance with respect to $\mathrm{Z}_{2}^{\pi}$ cancels, as for it, the pseudo-scalars present in the decomposition. Consequently, the proposed harmonic decomposition becomes simpler as the number of symmetries increases :

- Symmetry class $\left[\mathrm{Z}_{2}\right],\left[\mathrm{D}_{2}\right]$ : the full expression;

- Symmetry class $\left[\mathrm{D}_{4}\right]$ :

$$
\underset{\approx}{\mathrm{C}}=\underset{\approx}{\mathrm{H}^{2,2}}+\frac{\alpha^{2,2}}{2}{\underset{\mathrm{P}}{(2,2)}}^{(2)} \underset{\approx}{0,0} \mathrm{P}^{(2,0)}
$$

- Symmetry class $[\mathrm{O}(2)]$ :

$$
\underset{\approx}{\mathrm{C}}=\frac{\alpha^{2,2}}{2} \mathrm{P}^{(2,2)}+2 \alpha^{0,0} \mathrm{P}^{(2,0)}
$$

As discussed in $[76,10,26]$, these simplifications are related to relations between invariants of the studied tensor. In these references, it is indicated, for instance, how to distinguish $\left[\mathrm{Z}_{2}\right]$ symmetry class from the $\left[\mathrm{D}_{2}\right]$ one in terms of the harmonic tensors. For the sake of brevity, this aspect will not be discussed further here.

\section{Application to strain-Gradient elasticity}

In this last section we apply the proposed methodology to the fifth- and sixth-order elasticity tensors involved in strain-gradient elasticity. To proceed these decompositions, in accordance with our method, the first step consists in decomposing the state space $\mathbb{T}_{(i j) k}$ into a direct sum of $\mathrm{O}(2)$-irreducible spaces.

5.1. Decomposition of the state tensor space $\mathbb{T}_{(i j) k}$. The space $\mathbb{T}_{(i j) k}$ has the following harmonic structure:

$$
\mathbb{T}_{(i j) k} \simeq \mathbb{K}^{3} \oplus 2 \mathbb{K}^{1}
$$

Due to the multiplicity of $\mathbb{K}^{1}$ in the harmonic structure, the explicit harmonic decomposition is not uniquely defined [38]. The tensor in $\mathbb{K}^{3}$ is canonically defined but there are multiple possibilities concerning the decomposition of the vector parts. Among the different possibilities, some have more physical content than

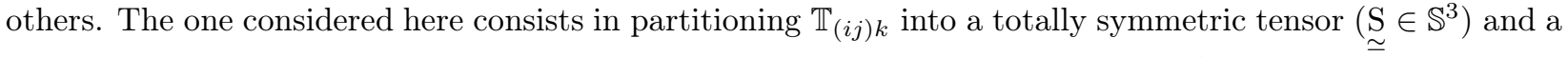
remainder, before proceeding to the harmonic decomposition of each part separately ${ }^{9}$. Another decomposition is proposed in Appendix C. The process of the decomposition is described in the following diagram:

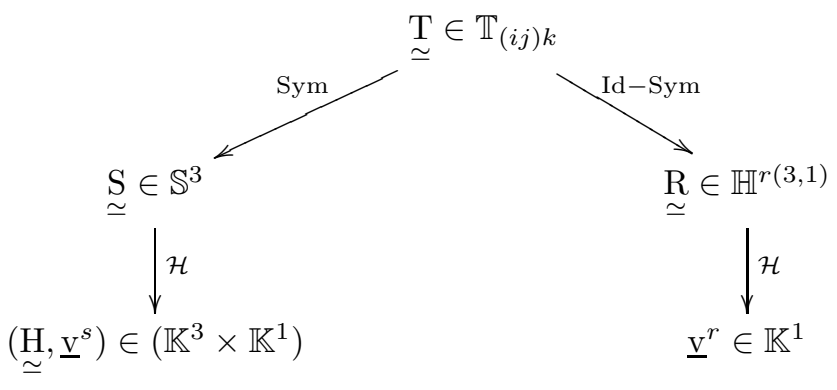

\footnotetext{
${ }^{8}$ The symmetry class of a tensor $\mathbf{T}$ is the conjugacy class, in $\mathrm{O}(2)$, of its symmetry group $G_{\mathbf{T}}:=\{\mathbf{g} \in \mathrm{O}(2), \mathbf{g} \star \mathbf{T}=\mathbf{T}\}$. The interested reader can find more details concerning this concept in the following references [36, 9].

${ }^{9}$ Unlike the decomposition of $\mathbb{T}_{(i j)}$ which was direct, the decomposition of $\mathbb{T}_{(i j) k}$ is two-step: 1) a splitting according to index symmetries of the tensor space; 2 ) the harmonic decomposition of the symmetric elementary part. This approach can be formalised (Schur-Weyl Harmonic Decomposition), however this is not the subject of this contribution.
} 
where Sym and $\mathcal{H}$ stand for the symmetrization and the harmonic decomposition processes, respectively. The space $\mathbb{H}^{r(3,1)}$ appearing in this diagram is defined as

$$
\mathbb{H}^{r(3,1)}:=\left\{\underset{\simeq}{\mathrm{T}} \in \mathbb{T}_{(i j) k} \mid T_{i j k}+T_{j k i}+T_{i k j}=0\right\}, \quad \operatorname{dim}\left(\mathbb{H}^{r(3,1)}\right)=2 .
$$

In the strain-gradient literature $\underset{\sim}{\mathrm{S}}$ describes the stretch-gradient part of the strain-gradient tensor, while $\underset{\simeq}{\mathrm{R}}$ is the rotation-gradient $[52,54]$.

We have the following result:

Theorem 5.1 (Harmonic decomposition of $\mathbb{T}_{(i j) k}$ ). There exists an $\mathrm{O}(2)$-equivariant isomorphism between $\mathbb{T}_{(i j) k}$ and $\mathbb{K}^{3} \oplus \mathbb{K}^{1} \oplus \mathbb{K}^{1}$ such that for $\underset{\simeq}{\mathrm{H}} \in \mathbb{K}^{3}$ and $\left(\underline{\mathrm{v}}^{s}, \underline{\mathrm{v}}^{r}\right) \in \mathbb{K}^{1} \times \mathbb{K}^{1}$,

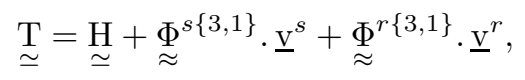

with $\left({\underset{\approx}{\Phi}}^{s\{3,1\}},{\underset{\approx}{\Phi}}^{r\{3,1\}}\right)$ the harmonic embeddings of the form:

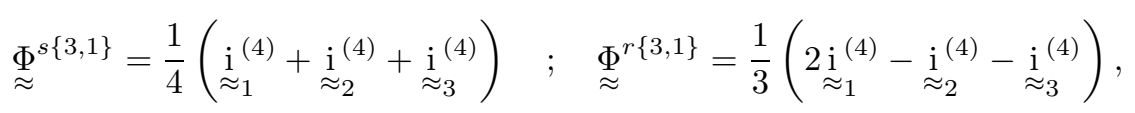

\begin{tabular}{|c|c|}
\hline $\mathbb{K}^{1}$ & $\mathbb{K}^{3}$ \\
\hline 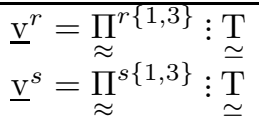 & $\underset{\sim}{\mathrm{H}}=\underset{\sim}{\mathrm{T}}-{\underset{\widetilde{\Phi}}{\Phi}}^{s\{3,1\}} \cdot \underline{\mathrm{v}}^{s}-\Phi_{\widetilde{\sim}}^{r\{3,1\}} \cdot \underline{\mathrm{v}}^{r}$ \\
\hline
\end{tabular}

in which $\left(\underset{\approx_{1}^{(}}{(4)},{\underset{\approx}{\mathrm{i}}}_{2}^{(4)},{\underset{\approx}{\mathrm{i}}}_{3}^{(4)}\right)$ are the fourth-order elementary isotropic tensors defined by Equation (1.1). Conversely, for any $\left.\underset{\simeq}{\mathrm{T}} \in \mathbb{T}_{(i j) k}, \underset{\simeq}{\mathrm{H}}, \underline{\mathrm{v}}^{s}, \underline{\mathrm{v}}^{r}\right) \in \mathbb{K}^{3} \times \mathbb{K}^{1} \times \mathbb{K}^{1}$ are defined from $\underset{\simeq}{\mathrm{T}}$ as follows:

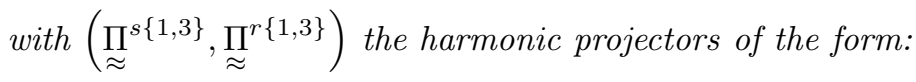

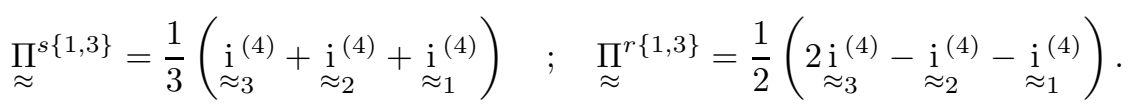

Remark 5.2. In the decomposition given by Equation (5.1), $\underline{\mathrm{v}}^{s}$ represents the vector part of the stretchgradient tensor and $\underline{\mathrm{v}}^{r}$ represents the vector part of the rotation-gradient tensor.

Remark 5.3. The decomposition is only valid for the Type II formulation of strain-gradient elasticity. To obtain the same expressions for the Type I formulation, that is for the decomposition of $\mathbb{T}_{i(j k)}$, the definition of $\underset{\approx}{\Phi^{r\{3,1\}} \text { must be replaced by the following } \underset{\approx}{\Phi^{\sharp}}[r 3,1\}}$

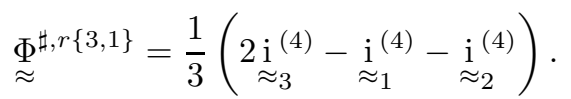

This substitution applied to all future formulas will make it possible to obtain decompositions of the operators of the Type I formulation from those of the Type II formulation.

Proof. The decomposition of $\underset{\simeq}{\mathrm{T}} \in \mathbb{T}_{(i j) k}$ is two-step: 1) $\underset{\simeq}{\mathrm{T}}$ is decomposed according to its index symmetries, then 2) each elementary part is decomposed into harmonic tensors.

Step 1: Index symmetry splitting

Any tensor $\underset{\simeq}{\mathrm{T}} \in \mathbb{T}_{(i j) k}$ can be decomposed into a complete symmetric tensor $\underset{\simeq}{\mathrm{S}} \in \mathbb{S}^{3}$ and a remainder $\underset{\simeq}{\mathrm{V}^{r}}$ as:

$$
\underset{\underline{\mathrm{T}}}{=} \underset{\underline{\mathrm{S}}}{\mathrm{S}}+{\underset{\mathrm{V}}{r}}^{r}
$$

with

$$
S_{i j k}=\frac{1}{3}\left(T_{i j k}+T_{i k j}+T_{j k i}\right) \quad \text { and } \quad V_{i j k}^{r}=\frac{1}{3}\left(2 T_{i j k}-T_{i k j}-T_{j k i}\right) .
$$

It is direct to check that the tensor $\underset{\simeq}{\mathrm{V}^{r}}$ belongs to $\mathbb{H}^{r(3,1)}$ and that the spaces $\mathbb{S}^{3}$ and $\mathbb{H}^{r(3,1)}$ are in direct sum. 


\section{Step 2: Harmonic decompositions}

Let us decompose $\underset{\sim}{\mathrm{S}} \in \mathbb{S}^{3}$ and $\underset{\sim}{\mathrm{V}^{r}} \in \mathbb{H}^{r(3,1)}$ into harmonic tensors. We consider this last case first. The following map

$$
v_{i}^{r} \mapsto V_{i j k}^{r}:=\frac{1}{3}\left(2 \delta_{i j} v_{k}^{r}-\delta_{i k} v_{j}^{r}-\delta_{j k} v_{i}^{r}\right)
$$

is an embedding of $\mathbb{K}^{1}$ into $\mathbb{H}^{r(3,1)}$, which can be rewritten in an intrinsic form as

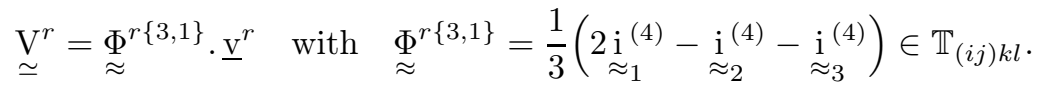

Since $\operatorname{dim}\left(\mathbb{H}^{r(3,1)}\right)=2=\operatorname{dim}\left(\mathbb{K}^{1}\right)$, there is no other harmonic embedding to consider. The expression of the projection from $\mathbb{H}^{r(3,1)}$ to $\mathbb{K}^{1}$ is obtained using Theorem 3.1:

$$
{\underset{\approx}{\pi}}^{r\{1,3\}}=\frac{1}{2}\left(\underset{\approx 3}{2 \mathrm{i}^{(4)}}-\underset{\approx 2}{\mathrm{i}^{(4)}}-\underset{\approx 1}{\mathrm{i}(4)}\right) \text {. }
$$

Now consider the decomposition of $\underset{\sim}{\mathrm{S}} \in \mathbb{S}^{3}$ into the sum of harmonic tensors. Using Lemma 2.1 the harmonic structure of $\mathbb{S}^{3}$ is known to be isomorphic to $\mathbb{K}^{3} \oplus \mathbb{K}^{1}$. Let $\mathbb{H}^{s(3,1)}$ be the space of totally symmetric third-order tensors which are orthogonal to tensors in $\mathbb{K}^{3}$ :

$$
\mathbb{H}^{s(3,1)}:=\left\{\underset{\simeq}{\mathrm{T}} \in \mathbb{S}^{3} \mid \underset{\simeq}{\forall \mathrm{H}} \in \mathbb{K}^{3}, \underset{\simeq}{\mathrm{H}}: \underset{\simeq}{\mathrm{T}}=0\right\} .
$$

Since $\operatorname{dim}\left(\mathbb{S}^{3}\right)=4$ and $\operatorname{dim}\left(\mathbb{K}^{3}\right)=2$, we deduce that $\operatorname{dim}\left(\mathbb{H}^{s(3,1)}\right)=2$. The following map:

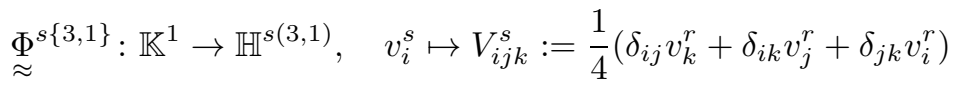

which can be rewritten in an intrinsic form as

$$
{\underset{\simeq}{\mathrm{V}}}^{s}=\underset{\approx}{\Phi^{s\{3,1\}}} \cdot \underline{\mathrm{v}}^{s} \quad \text { with } \quad \underset{\approx}{\Phi^{s\{3,1\}}}=\frac{1}{4}\left(\underset{\approx}{\mathrm{i}_{1}^{(4)}}+\underset{\approx 2}{\mathrm{i}(4)}+\underset{\approx}{\mathrm{i}^{(4)}}\right) \in \mathbb{S}^{4}
$$

is an embedding of $\mathbb{K}^{1}$ into $\mathbb{H}^{s(3,1)}$. A direct application of Theorem 3.1 provides the expression of the projection from $\mathbb{H}^{s(3,1)}$ onto $\mathbb{K}^{1}$ :

$$
\prod_{\approx}^{s\{1,3\}}=\frac{1}{3}\left(\underset{\approx}{\mathrm{i}_{3}^{(4)}}+\underset{\approx_{2}^{\mathrm{i}}}{(4)}+\underset{\mathrm{\sim}_{1}^{(4)}}{(4)}\right)
$$

The tensor $\underset{\simeq}{\mathrm{H}} \in \mathbb{K}^{3}$ is then directly deduced:

$$
\underset{\simeq}{\mathrm{H}}=\underset{\simeq}{\mathrm{S}}-\underset{\approx}{\Phi^{s\{3,1\}}} \cdot \underline{\mathrm{v}}^{s}
$$

From the embedding operators involved in Theorem 3.1 a family of projectors can be deduced.

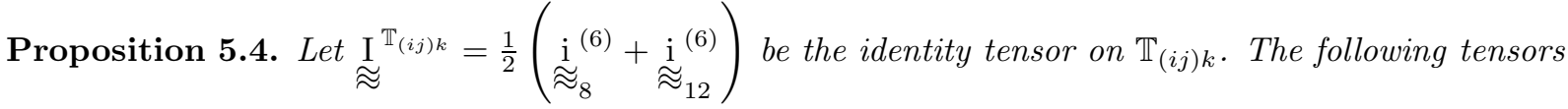

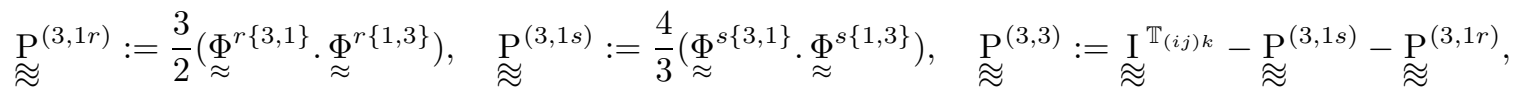

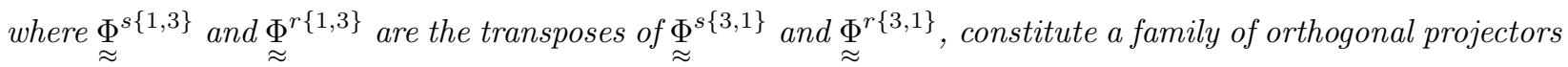
on $\mathbb{H}^{r(3,1)}, \mathbb{H}^{s(3,1)}$ and $\mathbb{K}^{3}$, respectively.

Proof. The multiplication table of the family $\left.\underset{\approx}{\left(\mathrm{P}^{(3,3)}\right.}, \underset{\approx}{\mathrm{P}^{(3,1 s)}}, \underset{\approx}{\mathrm{P}^{(3,1 r)}}\right)$ given below 


\begin{tabular}{|c|c|c|c|}
\hline$\vdots$ & $\stackrel{\mathrm{P}}{ }^{(3,3)}$ & $\mathrm{P}^{(3,1 s)}$ & $\mathrm{P}^{(3,1 r)}$ \\
\hline $\mathrm{P}^{(3,3)}$ & $\begin{array}{l}\mathrm{P}^{(3,3)} \\
\approx\end{array}$ & $\approx$ & $\approx$ \\
\hline$\stackrel{\mathrm{P}}{(3,1 s)}^{(3,1)}$ & $\underset{0}{\approx}$ & $\stackrel{\mathrm{P}}{(3,1 s)}^{(3)}$ & $\underset{0}{\approx}$ \\
\hline $\begin{array}{l}\mathrm{P}^{(3,1 r)} \\
\approx^{(3)}\end{array}$ & $\approx$ & $\underset{0}{\approx}$ & $\mathrm{P}^{(3,1 r)}$ \\
\hline
\end{tabular}

shows that $\left.\underset{\approx}{\mathrm{P}^{(3,3)}}, \underset{\approx}{\mathrm{P}^{(3,1 s)}}, \underset{\approx}{\mathrm{P}^{(3,1 r)}}\right)$ constitutes a family of orthogonal projectors with respect to the triple contraction.

Remark 5.5. To obtain the projectors for the Type I formulation, that is for the decomposition of $\mathbb{T}_{i(j k)}$, the definition of $\underset{\approx}{\mathrm{I}^{\mathbb{T}}}{ }^{(i j) k}$ must be replaced by the following one

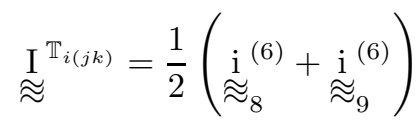

By combining this identity with $\underset{\approx}{\Phi^{\sharp}}[3,1\}$, the projectors $\left(\underset{\approx}{\mathrm{P}^{\sharp(3,3)}}, \underset{\approx}{\mathrm{P}^{(3,1 s)}}, \underset{\approx}{\mathrm{P}^{\sharp(3,1 r)}}\right)$ adapted to the Type I formulation are constructed.

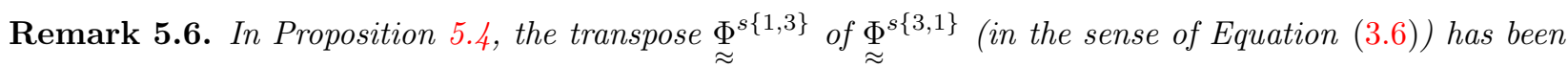

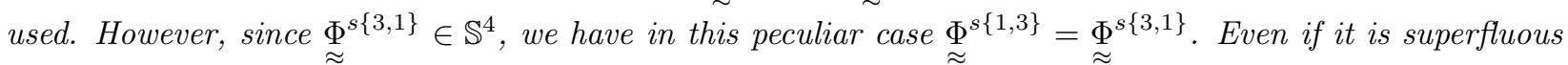

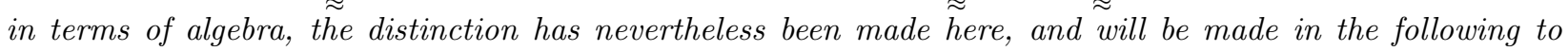
ensure consistency of notation in our different expressions.

Remark 5.7. From Lemma A.6, the tensors $\left.\underset{\approx}{\mathrm{P}^{(3,3)}}, \underset{\approx}{\mathrm{P}^{(3,1 s)}}, \underset{\approx}{\mathrm{P}^{(3,1 r)}}\right)$ can be considered as isotropic elasticity tensors in $\mathbb{E l a}_{6}$. Interpreted as elements of $\left(\mathbb{E} \mathrm{la}_{6},{ }^{(6)}\right)$, these tensors are associated to the following Gram matrix:

\begin{tabular}{|c|c|c|c|}
\hline $\begin{array}{l}(6) \\
\end{array}$ & $\begin{array}{l}\mathrm{P}^{(3,3)} \\
\approx\end{array}$ & $\stackrel{\mathrm{P}}{(3,1 s)}^{(3,1)}$ & $\stackrel{\mathrm{P}}{(3,1 r)}^{(3,)}$ \\
\hline $\begin{array}{l}\mathrm{P}^{(3,3)} \\
\approx\end{array}$ & 2 & 0 & 0 \\
\hline $\mathrm{P}^{(3,1 s)}$ & 0 & 2 & 0 \\
\hline $\mathrm{P}^{(3,1 r)}$ & 0 & 0 & 2 \\
\hline
\end{tabular}

on which it can be checked, with a slight abuse of notation, that $\underset{\approx}{\mathrm{P}}{ }^{(3, k)} \stackrel{(6)}{\mathrm{P}^{(3, k)}}=\operatorname{dim}\left(\mathbb{K}^{k}\right), k \in\{1,3\}$.

5.2. Decomposition of $\mathbb{E l a}_{6}$. We will begin by considering this case, since the construction of ClebschGordan Harmonic Decomposition of $\underset{\approx}{\mathrm{A}} \in \mathbb{E} \mathrm{Ela}_{6}$ follows almost directly the method previously introduced for $\mathrm{Ela}_{4}$.

5.2.1. Clebsch-Gordan Decomposition. In the following proposition, the tensors $\underset{\approx}{a^{1 s, 3}}$ and $\underset{\approx}{a^{1 r, 3}}$ will denote the transposes of the tensors $\underset{\approx}{\mathrm{a}^{3,1 s}}$ and $\underset{\approx}{\mathrm{a}^{3,1 r}}$, respectively, in the sense defined by Equation (3.6). 
Proposition 5.8. The tensor $\underset{\approx}{\mathrm{A}} \in \mathbb{E} \mathrm{la}_{6}$ admits the uniquely defined Intermediate Block Decomposition associated to the family of projectors $\left.\underset{\approx}{\mathrm{P}^{(3,3)}}, \underset{\mathrm{P}}{(3,1 s)}, \underset{\mathrm{P}}{(3,1 r)}\right)$ :

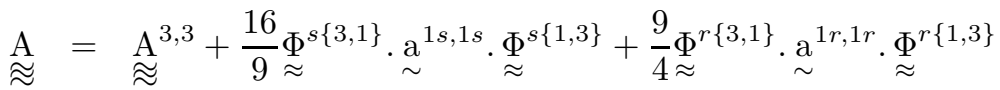

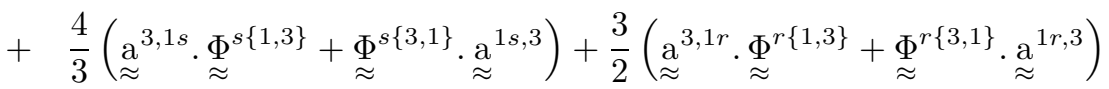

$$
\begin{aligned}
& +2\left(\underset{\approx}{\Phi^{s\{3,1\}}} \cdot{\underset{\sim}{\mathrm{a}}}^{1 s, 1 r} \cdot \underset{\approx}{\Phi^{r\{1,3\}}}+\underset{\approx}{\Phi^{r\{3,1\}}} \cdot{\underset{\sim}{\mathrm{a}}}^{1 r, 1 s} \cdot{\underset{\approx}{\Phi}}^{s\{1,3\}}\right)
\end{aligned}
$$

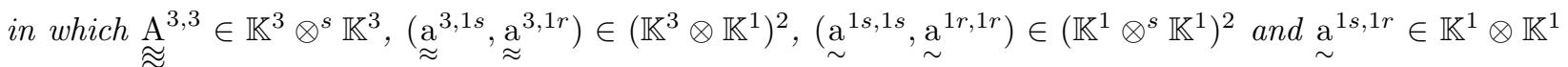

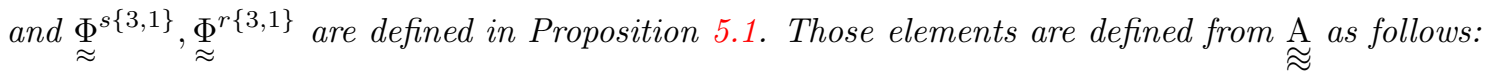

\begin{tabular}{|c|c|c|}
\hline $\mathbb{T}^{2}$ & $\mathbb{T}^{4}$ & $\mathbb{T}^{6}$ \\
\hline 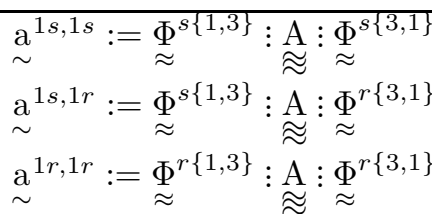 & 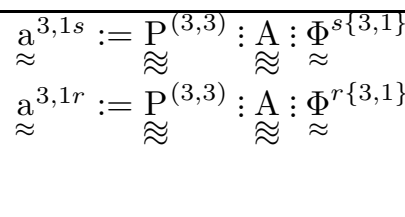 & $\stackrel{\mathrm{A}}{\text { A }}^{3,3}:=\underset{\approx}{\mathrm{P}}{ }^{(3,3)} \vdots \underset{\approx}{\mathrm{A}}: \underset{\approx}{\mathrm{P}^{(3,3)}}$ \\
\hline
\end{tabular}

Proof. The proof follows the steps detailed in the proof of Proposition 4.3, only the main points will be summed up here. Starting from the decomposition introduced in Theorem 5.1, any $\underset{\simeq}{\mathrm{T}} \in \mathbb{T}_{(i j) k}$ decomposes as follows:

$$
\underset{\simeq}{\mathrm{T}}=\underset{\simeq}{\mathrm{H}}+\underset{\simeq}{\mathrm{V}^{s}}+\underset{\simeq}{\mathrm{V}^{r}}
$$

Using the projectors $\left(\underset{\approx}{\mathrm{P}^{(3,3)}}, \underset{\approx}{\mathrm{P}^{(3,1 s)}}, \underset{\approx}{\mathrm{P}^{(3,1 r)}}\right)$ and following the method used in the proof of Proposition 4.3, the constitutive law can be brought to the following matrix form:

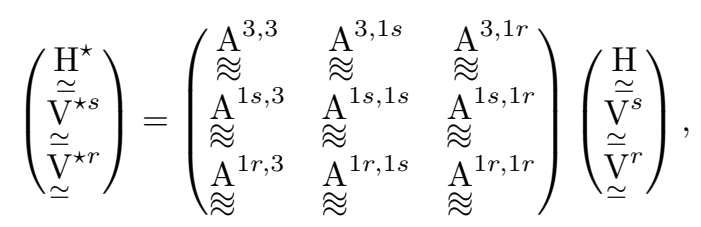

where the quantities are defined as:

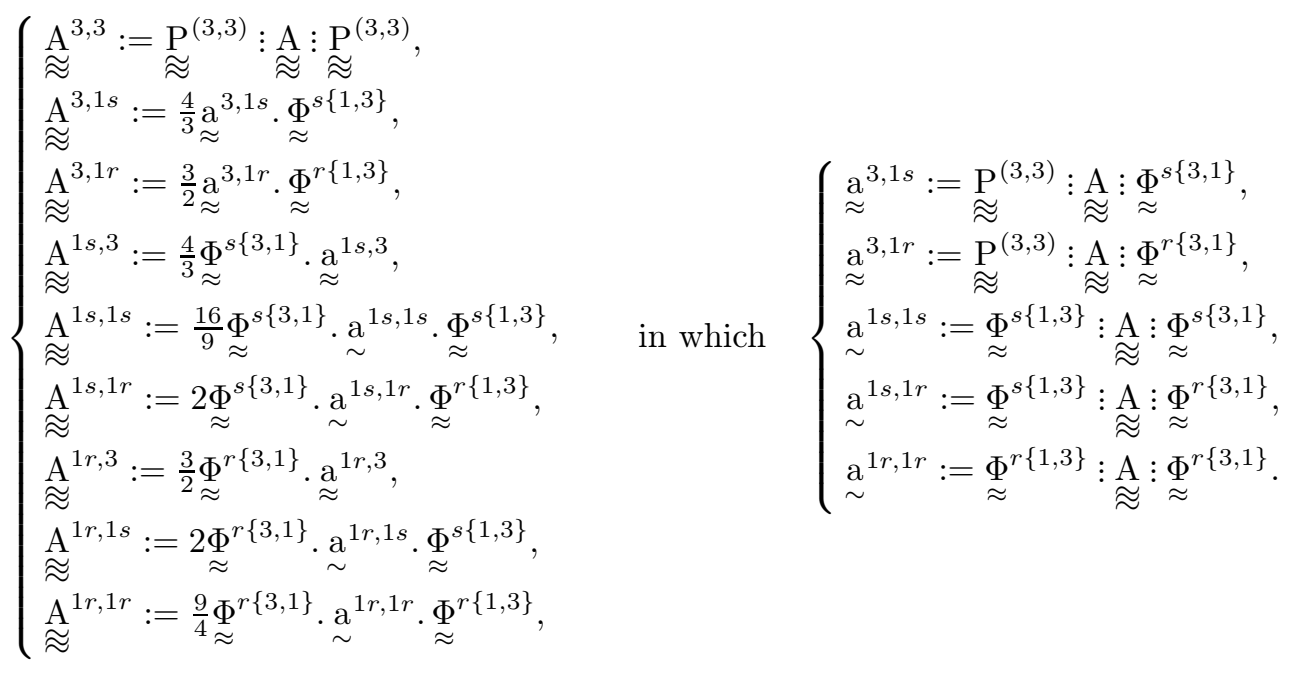


Using matrix notation, we have

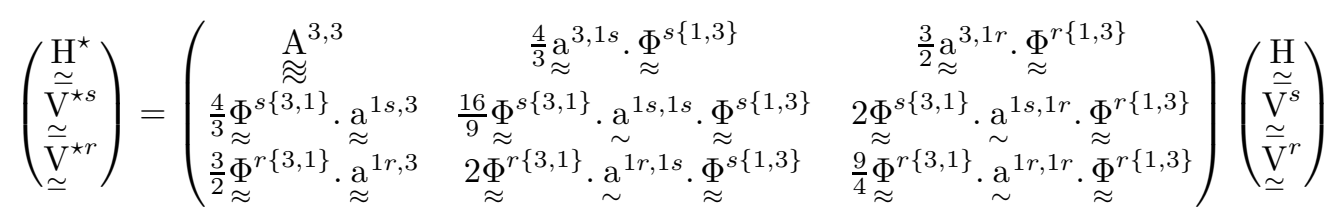

5.2.2. Harmonic Decomposition. In the Intermediate Block Decomposition of $\underset{\approx}{\mathrm{A}}$ the non-harmonic tensors belong to 4 different spaces:

- $\underset{\approx}{\mathrm{A}^{3,3}} \in \mathbb{K}^{3} \otimes^{s} \mathbb{K}^{3} \simeq \mathbb{K}^{6} \oplus \mathbb{K}^{0}$

- $\left(\underset{\widetilde{a^{3}}}{3,1 s}, \underset{\sim}{\mathrm{a}^{3,1 r}}\right) \in \mathbb{K}^{3} \otimes \mathbb{K}^{1} \simeq \mathbb{K}^{4} \oplus \mathbb{K}^{2}$;

- $\left(\underset{\sim}{\mathrm{a}^{1 s, 1 s}}, \underset{\sim}{\mathrm{a}^{1 r, 1 r}}\right) \in \mathbb{K}^{1} \otimes^{s} \mathbb{K}^{1} \simeq \mathbb{K}^{2} \oplus \mathbb{K}^{0}$

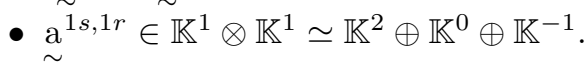

Their harmonic decompositions are provided in Appendix B, the associated results are the following:

- Tensors $\underset{\approx}{\mathrm{A}^{3,3}} \in \mathbb{K}^{3} \otimes^{s} \mathbb{K}^{3}$ admit the uniquely defined harmonic decomposition

$$
\underset{\mathrm{A}}{\approx} \approx \underset{2}{\approx} \underset{\approx}{\mathrm{H}}+\frac{\alpha}{2} \mathrm{P}^{(3,3)}, \text { where } \underset{\approx}{\mathrm{H}} \in \mathbb{K}^{6} \text { and } \alpha \in \mathbb{K}^{0}
$$

Conversely,

$$
\alpha=\underset{\mathrm{A}^{3,3}}{\approx} \stackrel{(6)}{\underset{\mathrm{P}}{(3,3)}} \text { and } \underset{\approx}{\mathrm{H}}=\underset{\approx}{\mathrm{A}^{3,3}}-\frac{\alpha}{2} \underset{\mathrm{P}^{(3,3)}}{\approx}
$$

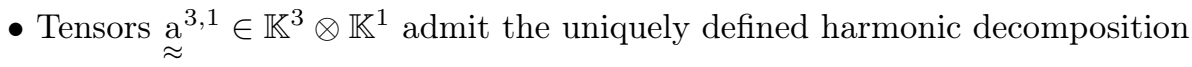

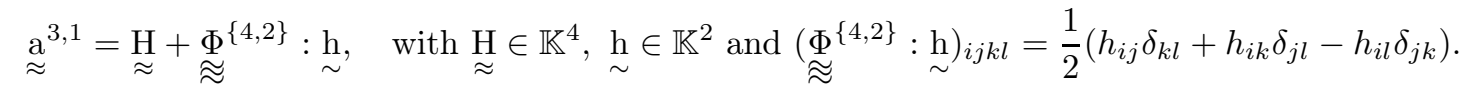

Conversely,

$$
\underset{\sim}{\mathrm{h}}=\operatorname{tr}_{14} \underset{\approx}{\mathrm{a}^{3,1}} \text { and } \underset{\approx}{\mathrm{H}}=\underset{\approx}{\mathrm{a}^{3,1}}-\underset{\approx}{\underset{\approx}{\{4,2\}}: \mathrm{h} .}
$$

The notation $\operatorname{tr}_{i j}$ indicates that the contraction should be done on the $i$ th and $j$ th indices.

- Tensors $\underset{\sim}{a^{1,1}} \in \mathbb{K}^{1} \otimes \mathbb{K}^{1}$ admit the uniquely defined harmonic decomposition

$$
\underset{\sim}{\mathrm{a}}{ }^{1,1}=\underset{\sim}{\mathrm{d}}+\frac{\beta}{2} \epsilon+\frac{\alpha}{2} \mathrm{i}_{\sim}^{(2)}, \text { with } \underset{\sim}{\mathrm{d}} \in \mathbb{K}^{2},(\alpha, \beta) \in \mathbb{K}^{0} .
$$

Conversely,

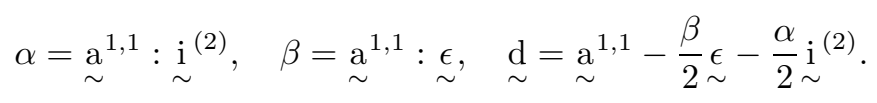

In the following proposition, the notation $\mathbf{T}^{T_{\alpha, \beta}}$ indicates a generalized transposition operation in which, in components, the first $\alpha$ indices are permuted with the last $\beta$ ones.

Proposition 5.9 (Clebsch-Gordan Harmonic Decomposition of $\underset{\approx}{\mathrm{A}} \in \mathbb{E} \mathrm{la}_{6}$ ). The tensor $\underset{\approx}{\mathrm{A}} \in \mathbb{E} \mathrm{la}_{6}$ admits the uniquely defined Clebsch-Gordan Harmonic Decomposition associated to the family of projectors $\left(\mathrm{P}^{(3,3)}, \mathrm{P}^{(3,1 s)}, \mathrm{P}^{(3,1 r)}\right)$ 


$$
\begin{aligned}
& \underset{\approx}{\mathrm{A}}=\underset{\approx}{\mathrm{H}^{3,3}}+\frac{4}{3}\left(\underset{\approx}{\mathrm{H}^{3,1 s}} \cdot \underset{\approx}{\Phi^{s\{1,3\}}}+\underset{\approx}{\Phi^{s\{3,1\}}} \cdot \underset{\approx}{\mathrm{H}^{3,1 s}}\right)+\frac{3}{2}\left(\underset{\approx}{\mathrm{H}^{3,1 r}} \cdot \underset{\approx}{\Phi^{r\{1,3\}}}+\underset{\approx}{\Phi^{r\{3,1\}}} \cdot \underset{\approx}{\mathrm{H}^{3,1 r}}\right)
\end{aligned}
$$

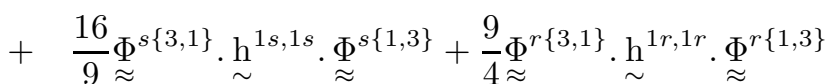

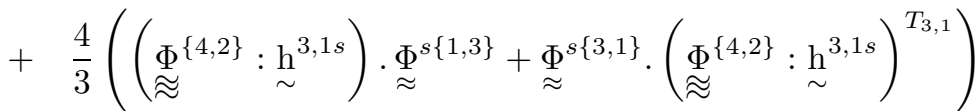

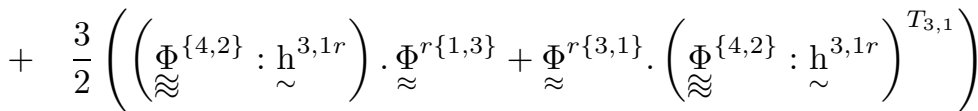

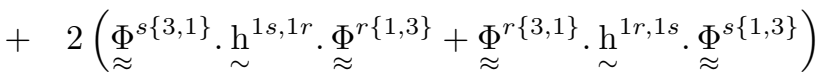

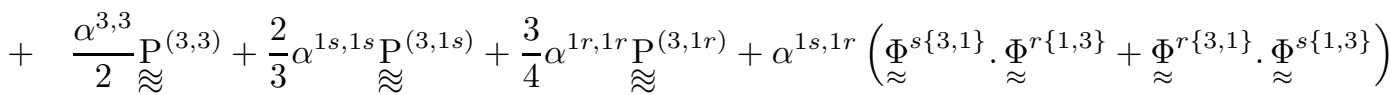

$$
\begin{aligned}
& +\beta^{1 s, 1 r}\left(\underset{\approx}{\Phi^{s\{3,1\}}} \cdot \underset{\sim}{\epsilon} \underset{\approx}{\Phi^{r\{1,3\}}}-\underset{\approx}{\Phi^{r\{3,1\}}} \cdot \underset{\sim}{\epsilon} \cdot \underset{\approx}{\Phi^{s\{1,3\}}}\right),
\end{aligned}
$$

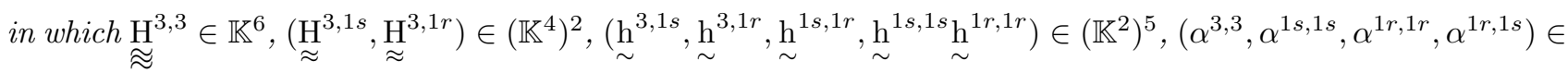
$\left(\mathbb{K}^{0}\right)^{4}$ and $\beta^{1 r, 1 s} \in \mathbb{K}^{-1}$. Those elements are defined from $\underset{\approx}{\mathrm{A}}$ as follows:

\begin{tabular}{|c|c|c|c|c|}
\hline $\mathbb{K}^{-1}$ & $\mathbb{K}^{0}$ & $\mathbb{K}^{2}$ & $\mathbb{K}^{4}$ & $\mathbb{K}^{6}$ \\
\hline$\beta^{1 s, 1 r}={\underset{\sim}{a^{1 s, 1 r}}: \underset{\sim}{\epsilon}}^{\epsilon}$ & 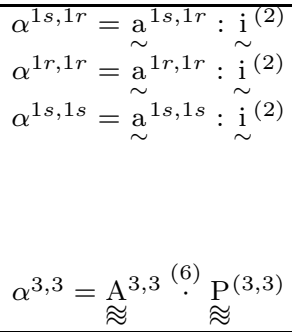 & 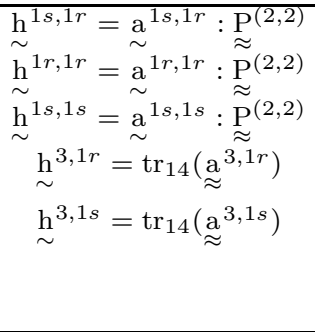 & 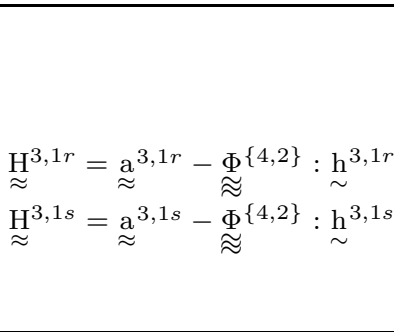 & $\underset{\approx}{\mathrm{H}^{3,3}}=\underset{\approx}{\mathrm{A}^{3,3}}-\frac{\alpha^{3,3}}{2} \underset{\approx}{\stackrel{\mathrm{P}}{(3,3)}}$ \\
\hline
\end{tabular}

in which the intermediate quantities are defined in Proposition 5.8.

Remark 5.10. Following Remarks 5.3 and 5.5, the formula for strain-gradient elasticity expressed within type I formulation is directly obtained by substituting $\underset{\approx}{\stackrel{\mathrm{P}}{\sharp(3,3)}, \underset{\approx}{\mathrm{P}}}$ $\Phi^{r\{3,1\}}$ in the given formula.

5.3. Link with symmetry classes. As demonstrated in [7, 9], the space $\mathbb{E} l_{6}$ is divided into the 8 following symmetry classes:

$$
\mathfrak{I}\left(\mathbb{E l a} a_{6}\right)=\left\{\left[\mathrm{Z}_{2}\right],\left[\mathrm{D}_{2}\right],\left[\mathrm{Z}_{4}\right],\left[\mathrm{D}_{4}\right],\left[\mathrm{Z}_{6}\right],\left[\mathrm{D}_{6}\right],[\mathrm{SO}(2)],[\mathrm{O}(2)]\right\}
$$

These symmetry classes can be linked as shown in the following diagram:

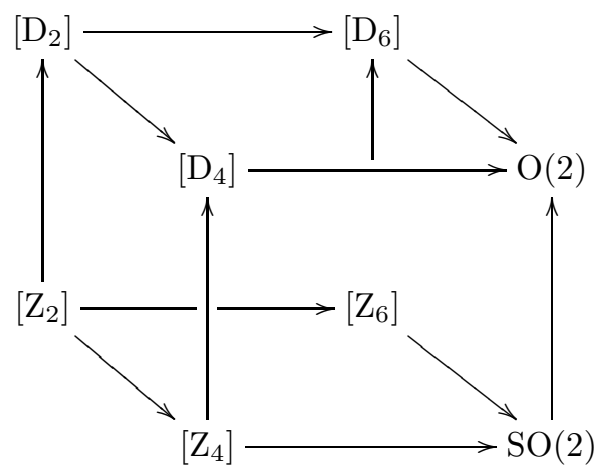

For the high-symmetry classes $\left[\mathrm{Z}_{6}\right],\left[\mathrm{D}_{6}\right],[\mathrm{SO}(2)],[\mathrm{O}(2)]$ the harmonic decomposition reduces to 
- Symmetry class $\left[\mathrm{Z}_{6}\right]$ :

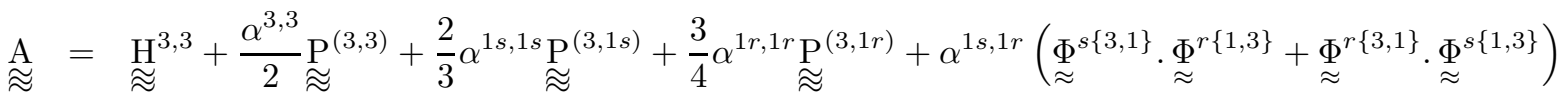

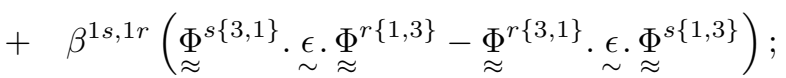

- Symmetry class $\left[\mathrm{D}_{6}\right]$ :

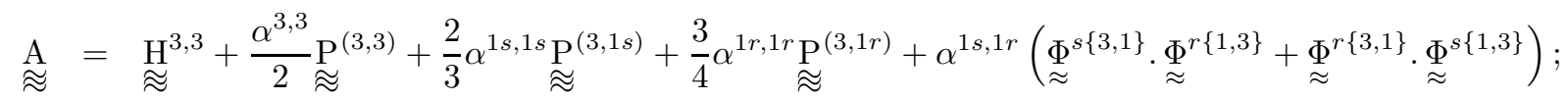

- Symmetry class $[\mathrm{SO}(2)]$ :

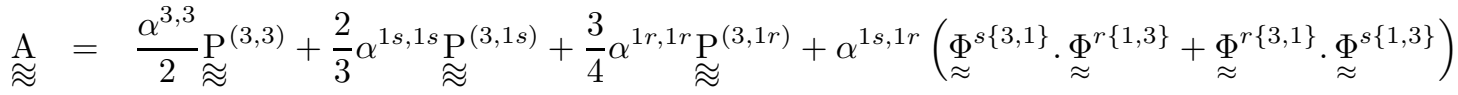

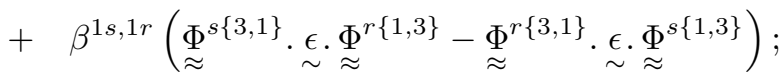

- Symmetry class $[\mathrm{O}(2)]$ :

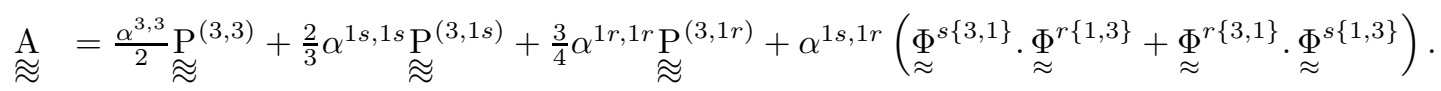

In order to provide the harmonic decomposition for the other cases without ambiguity, additional tools, not discussed in this paper, are needed. We therefore defer this point to a future contribution.

5.4. Decomposition of $\mathbb{E}$ las. In this last subsection, the Clebsch-Gordan Harmonic Decomposition of $\underset{\approx}{\mathrm{M}} \in$ $\mathbb{E}$ la $_{5}$ is provided. Since $\mathbb{E} l_{5} \simeq \mathcal{L}\left(\mathbb{T}_{(i j) k}, \mathbb{T}_{(i j)}\right)$, two different state tensor spaces are involved for constructing the decomposition.

\subsubsection{Clebsch-Gordan Decomposition.}

Proposition 5.11. The tensor $\underset{\approx}{\mathrm{M}} \in \mathbb{E} \mathrm{la}_{5}$ admits the uniquely defined Intermediate Block Decomposition associated to the family of projectors $\underset{\approx}{\mathrm{P}^{(3,3)}} \underset{\approx}{\mathrm{P}}\left(\mathrm{P}^{(3,1 s)}, \underset{\approx}{\mathrm{P}^{(3,1 r)}}, \underset{\approx}{\mathrm{P}^{(2,2)}}, \underset{\approx}{\mathrm{P}^{(2,0)}}\right)$ :

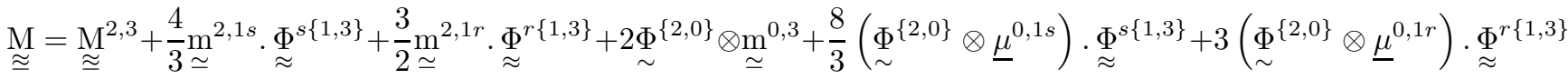

\begin{tabular}{|c|c|c|}
\hline $\mathbb{T}^{1}$ & $\mathbb{T}^{3}$ & $\mathbb{T}^{5}$ \\
\hline $\begin{array}{l}\underline{\mu}^{0,1 s}:={\underset{\sim}{\Phi}}^{\{0,2\}}: \underset{\approx}{\mathrm{M}}: \underset{\approx}{\Phi^{s\{3,1\}}} \\
\underline{\mu}^{0,1 r}:={\underset{\sim}{ }}^{\{0,2\}}: \underset{\approx}{\mathrm{M}}: \underset{\approx}{\Phi^{r\{3,1\}}}\end{array}$ & 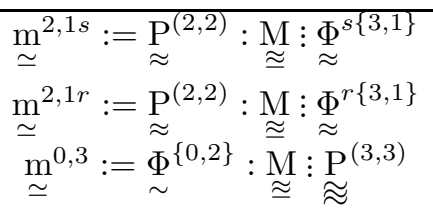 & $\stackrel{\mathrm{M}}{ }^{2,3}:={\underset{\approx}{\mathrm{P}}}^{(2,2)}: \underset{\approx}{\mathrm{M}}: \mathrm{P}^{(3,3)}$ \\
\hline
\end{tabular}

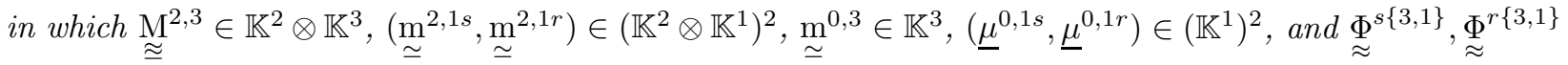
and ${\underset{\sim}{\Phi}}^{\{0,2\}}$ are defined, respectively, in Propositions 5.1 and 4.2. Those elements are defined from $\underset{\approx}{\mathrm{M}}$ as follows:

Proof. The proof follows the steps detailed in the proof of Proposition 4.3, only the main points will be summed up here. The constitutive law can be expressed in matrix notation as follows:

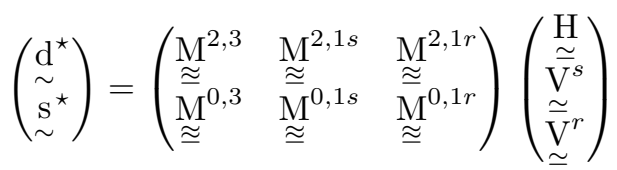


with

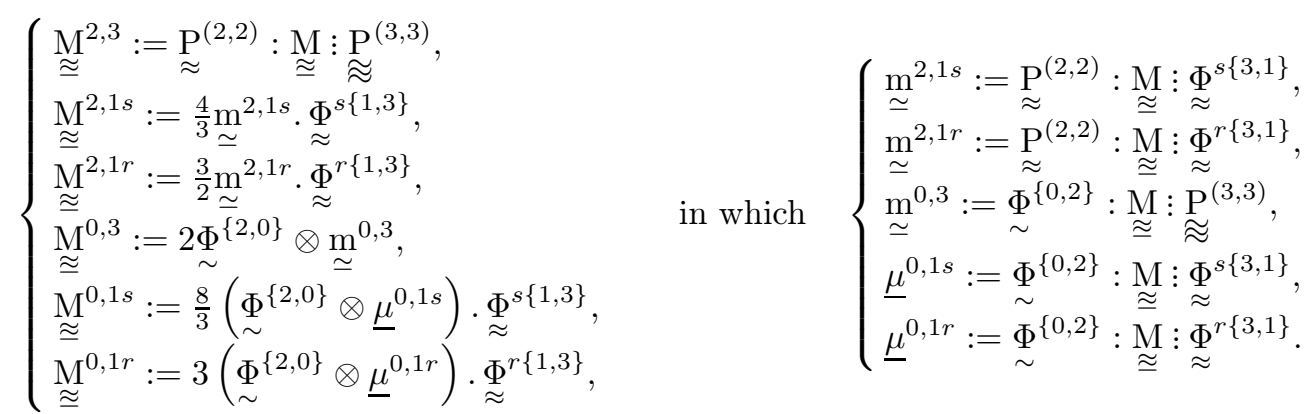

In matrix form, we have

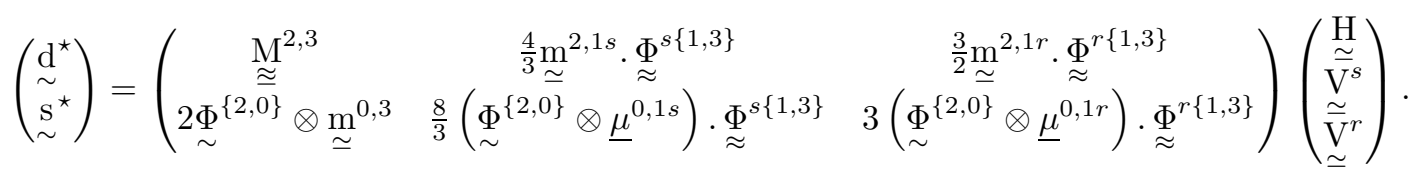

By construction, it can directly be checked that

$$
\underset{\widetilde{\mathrm{M}}}{2,3} \in \mathbb{K}^{2} \otimes \mathbb{K}^{3},\left(\underset{\simeq}{\mathrm{m}^{2,1 s}}, \underline{\simeq}^{2,1 r}\right) \in \mathbb{K}^{2} \otimes \mathbb{K}^{1}, \underline{\simeq}^{0,3} \in \mathbb{K}^{0} \otimes \mathbb{K}^{3}, \operatorname{and}\left(\underline{\mu}^{0,1 s}, \underline{\mu}^{0,1 r}\right) \in \mathbb{K}^{0} \otimes \mathbb{K}^{1} .
$$

5.4.2. Harmonic Decomposition. In the Intermediate Block Decomposition of $\underset{\approx}{\mathrm{M}}$ the only non-harmonic tensors are $\underset{\widetilde{\sim}}{\mathrm{M}^{2,3}} \in \mathbb{K}^{2} \otimes \mathbb{K}^{3}$ and $\left.\underset{\simeq}{\mathrm{m}^{2,1 s}}, \underset{\simeq}{\mathrm{m}^{2,1 r}}\right)$ which belong to $\mathbb{K}^{2} \otimes \mathbb{K}^{1}$. Their harmonic decompositions are provided in Appendix B, the associated results are the following:

- Tensors $\mathrm{m}^{2,1} \in \mathbb{K}^{2} \otimes \mathbb{K}^{1}$ admit the uniquely defined harmonic decomposition

$$
\left.{\underset{\mathrm{m}}{2}}^{2,1}=\underset{\simeq}{\mathrm{H}}+\underset{\approx}{\Phi^{\{3,1\}}} \cdot \underline{\mathrm{v}}, \quad \text { where } \underset{\simeq}{\mathrm{H}} \in \mathbb{K}^{3}, \underline{\mathrm{v}} \in \mathbb{K}^{1}, \text { and }{\underset{\approx}{\Phi}}^{\{3,1\}} \cdot \underline{\mathrm{v}}\right)_{i j k}=\frac{1}{2}\left(v_{i} \delta_{j k}+v_{j} \delta_{i k}-v_{k} \delta_{i j}\right) .
$$

Conversely,

$$
\underline{\mathrm{v}}=\operatorname{tr}_{13} \underset{\simeq}{\mathrm{m}^{2,1}} \text { and } \underset{\simeq}{\mathrm{H}}={\underset{\simeq}{\mathrm{m}}}^{2,1}-\underset{\approx}{\Phi}{ }^{\{3,1\}} \cdot \underline{\mathrm{v}} .
$$

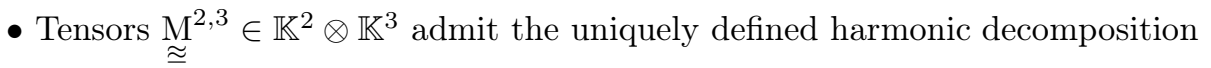

$$
\underset{\approx}{\mathrm{M}^{2,3}}=\underset{\approx}{\mathrm{H}}+\underset{\approx}{\Phi} \Phi^{\{5,1\}} \cdot \underline{\mathrm{v}}, \text { where } \underset{\approx}{\mathrm{H}} \in \mathbb{K}^{5}, \underline{\mathrm{v}} \in \mathbb{K}^{1}
$$

and

$$
\begin{aligned}
4\left(\stackrel{\Phi}{\Phi}^{\{5,1\}} \cdot \underline{\mathrm{v}}\right)_{i j k l m} & =v_{i}\left(\delta_{j k} \delta_{l m}-\delta_{j l} \delta_{k m}-\delta_{j m} \delta_{k l}\right)-v_{j} \delta_{i m} \delta_{k l} \\
& +v_{k}\left(-2 \delta_{i j} \delta_{l m}+\delta_{i l} \delta_{j m}+2 \delta_{i m} \delta_{j l}\right)+v_{l} \delta_{i k} \delta_{j m}+v_{m} \delta_{i j} \delta_{k l}
\end{aligned}
$$

Conversely,

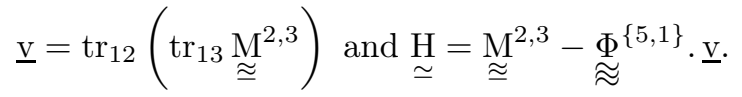

Using these results we obtain:

Proposition 5.12 (Clebsch-Gordan Harmonic Decomposition of $\underset{\approx}{\mathrm{M}} \in \mathbb{E}$ las $)$. The tensor $\underset{\approx}{\mathrm{M}} \in \mathbb{E} \mathrm{la}_{5}$ admits the uniquely defined Clebsch-Gordan Harmonic Decomposition associated to the family of projectors $\left(\underset{\mathrm{P}}{(3,3)}, \underset{\approx}{\mathrm{P}}{ }^{(3,1 s)}, \underset{\approx}{\mathrm{P}}{ }^{(3,1 s)}, \underset{\approx}{\mathrm{P}^{(2,2)}}, \underset{\approx}{\mathrm{P}^{(2,0)}}\right)$

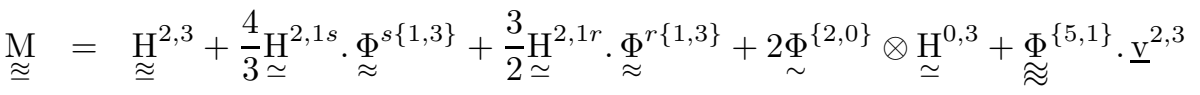

$$
\begin{aligned}
& +\frac{4}{3}\left({\underset{\approx}{\Phi}}^{\{3,1\}} \cdot \underline{\underline{v}}^{2,1 s}\right) \cdot{\underset{\approx}{\Phi}}^{s\{1,3\}}+\frac{3}{2}\left({\underset{\approx}{\Phi}}^{\{3,1\}} \cdot \underline{\mathrm{v}}^{2,1 r}\right) \cdot{\underset{\approx}{\Phi}}^{r\{1,3\}}+\frac{8}{3}\left({\underset{\sim}{\Phi}}^{\{2,0\}} \otimes \underline{\mu}^{0,1 s}\right) \cdot{\underset{\approx}{\Phi}}^{s\{1,3\}} \\
& +3\left(\underline{\sim}^{\{2,0\}} \otimes \underline{\mu}^{0,1 r}\right) \cdot \Phi^{\Phi}{ }^{r\{1,3\}}
\end{aligned}
$$


in which $\left.\underset{\widetilde{\mathrm{H}}}{\mathrm{H}}{ }^{, 3} \in \mathbb{K}^{5},{\underset{\underline{\mathrm{H}}}{ }}^{2,1 s}, \underline{\mathrm{H}}^{2,1 r}, \underline{\mathrm{H}}^{0,3}\right) \in\left(\mathbb{K}^{3}\right)^{3},\left(\underline{\mathrm{v}}^{2,3}, \underline{\mathrm{v}}^{2,1 s}, \underline{\mathrm{v}}^{2,1 r}, \underline{\mathrm{v}}^{0,1 s}, \underline{\mathrm{v}}^{0,1 r}\right) \in\left(\mathbb{K}^{1}\right)^{5}$. Those elements are defined from $\underset{\approx}{\mathrm{M}}$ as follows:

\begin{tabular}{|c|c|c|}
\hline $\mathbb{K}^{1}$ & $\mathbb{K}^{3}$ & $\mathbb{K}^{5}$ \\
\hline $\begin{array}{c}\underline{\mathrm{v}}^{0,1 r}=\underline{\mu}^{0,1 r} \\
\underline{\mathrm{v}}^{0,1 s}=\bar{\mu}^{0,1 s} \\
\underline{\mathrm{v}}^{2,1 r}=\underline{\mathrm{m}}^{2,1 r}: \stackrel{\mathrm{i}}{ }^{(2)} \\
\underline{\mathrm{v}}^{2,1 s}={\underset{\mathrm{m}}{ }}^{2,1 s}:{\underset{\mathrm{i}}{ }}^{(2)} \\
\underline{\mathrm{v}}^{2,3}=\operatorname{tr}_{12}\left(\operatorname{tr}_{13}\left({\underset{\widetilde{\mathrm{M}}}{ }}^{2,3}\right)\right)\end{array}$ & 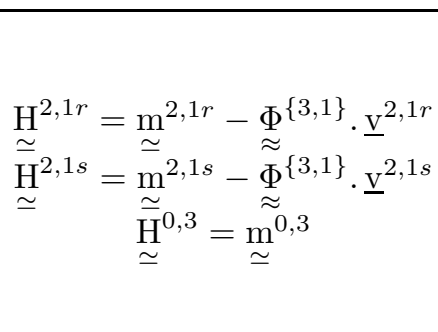 & ${\underset{\mathrm{H}}{\mathrm{H}}}^{2,3}={\underset{\approx}{\mathrm{M}}}^{2,3}-{\underset{\Phi}{\Phi}}^{\{5,1\}} \cdot \underline{\mathrm{v}}^{2,3}$ \\
\hline
\end{tabular}

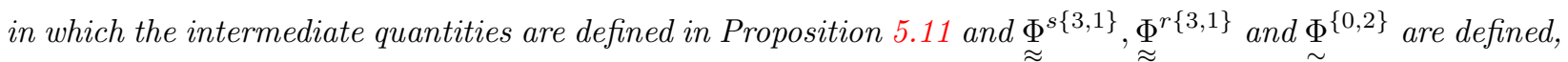
respectively, in Propositions 5.1 and 4.2.

Remark 5.13. Following Remarks 5.3 and 5.5, the formula for strain-gradient elasticity expressed within

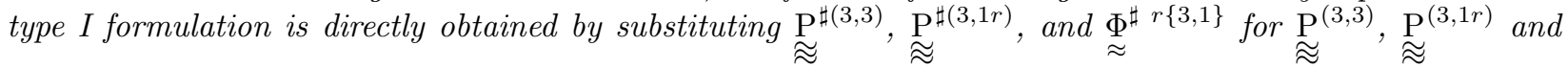
${\underset{\Phi}{r\{3,1\}}}^{r \text { in the given formula. }}$

5.5. Link with symmetry classes. As demonstrated in $[8,9]$, the space Elas is divided into the 6 following symmetry classes:

$$
\mathfrak{I}\left(\mathbb{E l a}_{5}\right)=\left\{\mathbb{1},\left[\mathrm{Z}_{2}^{\pi}\right],\left[\mathrm{Z}_{3}\right],\left[\mathrm{D}_{3}\right],\left[\mathrm{D}_{5}\right],[\mathrm{O}(2)]\right\}
$$

These symmetry classes can be linked as shown in the following diagram:

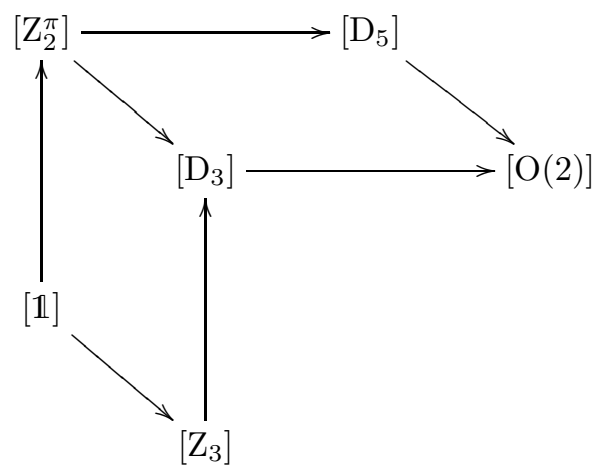

For the high-symmetries classes $\left[\mathrm{D}_{5}\right],[\mathrm{O}(2)]$ the harmonic decomposition simplifies into

- Symmetry class $\left[\mathrm{D}_{5}\right]$ :

$$
\underset{\approx}{\mathrm{M}}=\underset{\approx}{\mathrm{H}^{2,3}}
$$

- Symmetry class $[\mathrm{O}(2)]$ :

$$
\underset{\cong}{\mathrm{M}}=\underset{\approx}{0}
$$

In order to provide the harmonic decomposition for the other cases without ambiguity, additional tools, not discussed in this paper, are needed. We therefore defer this point to a future contribution.

Remark 5.14. As demonstrated in [8], the set of symmetry classes for the complete constitutive law, that is of a triplet $\mathcal{E}:=(\underset{\approx}{\mathrm{C}}, \underset{\approx}{\mathrm{M}} \underset{\underset{\approx}{\mathrm{A}}}{\mathrm{\approx}}) \in \mathcal{S}$ grd, is not the union of the symmetry classes of each tensor space considered separately. It can be shown, for example, that $\left[\mathrm{Z}_{5}\right]$ is a symmetry class of the complete constitutive law, although it is not a symmetry classes of any individual tensor in the triplet $\mathcal{E}$. We therefore refer interested readers to the reference [8] to see how to correctly combine the different results for each of the 14 symmetry classes of the 2D strain-gradient elasticity. 


\section{Conclusion}

In this paper the harmonic decomposition of the constitutive tensors appearing in the 2D Mindlin's straingradient elasticity has been investigated. Since no method available in the literature was considered satisfactory for the harmonic decomposition of higher-order tensors, a new harmonic decomposition, referred to here as the Clebsch-Gordan Harmonic Decomposition, was proposed. The main results of the paper are two-fold:

- the explicit 2D Clebsh-Gordan harmonic decompositions of:

- the fifth-order coupling tensor of strain-gradient elasticity;

- the sixth-order elasticity tensor of strain-gradient elasticity.

- the algorithm for the explicit Clebsh-Gordan harmonic decomposition for bidimensional tensors.

The Clebsch-Gordan algorithm is two-step and based on the explicit construction of the Clebsch-Gordan harmonic products. This approach, which shares some ideas with the one introduced by Zou in [82], allows one to easily obtain orthogonal harmonic decompositions of higher-order tensors. Since the Clebsch-Gordan construction generates a new harmonic decomposition from a known one, the procedure can be iterated to obtain harmonic decompositions of arbitrary order tensors. The approach developed here in the $2 \mathrm{D}$ situation can be extended without any problem to the harmonic decomposition of $3 \mathrm{D}$ tensors. The study of this extension will be the object of a future contribution.

As we have already mentioned, the proposed method for decomposing tensors that we have introduced is very general and its applicability is by no means restricted to strain-gradient elasticity. We believe that this method will find interesting applications beyond the one considered in the present contribution.

\section{ACKNOWLEDGEMENTS}

We thank Marc Olive for the multiple scientific discussions throughout the redaction of the manuscript. The first and the second authors acknowledge the support of the French Agence Nationale de la Recherche (ANR), under grant ANR-17-CE08-0039 (project ArchiMatHOS).

\section{Appendix A. Proofs of Theorem 3.1 And Proposition 3.2}

This appendix is devoted to the formulation and the proofs of Lemmas required to demonstrate Theorem 3.1 and Proposition 3.2. The main results of this appendix are Proposition A.4 and Lemmas A.6 and A.4. The other Propositions are intermediate results necessary to demonstrate them.

Proposition A.1. If $n$ and $k$ are of the same parity, $\Phi^{\{n, k\}}$ is an non-null isotropic tensor of order $k+n$, i.e.

$$
\Phi^{\{n, k\}}=\sum_{i} \lambda_{i} \mathbf{i}_{i}^{(n+k)}
$$

otherwise $\Phi^{\{n, k\}}$ is the null tensor.

Proof. $\Phi^{\{n, k\}}$ is an $\mathrm{O}(2)$-equivariant linear map between tensor spaces of order $n$ and $k$ with $n \geq k$, consequently:

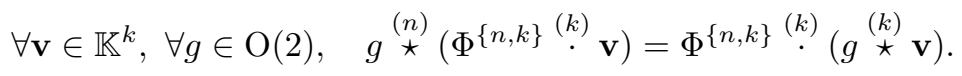

Using the change of variables $\mathbf{v}=g^{T} \stackrel{(k)}{\cdot} \mathbf{v}^{\star}$, we have

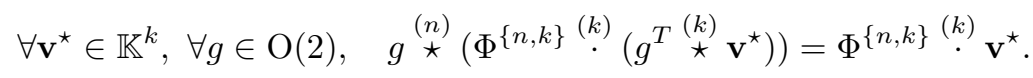

Moreover,

$$
\begin{aligned}
& g \stackrel{(n)}{\star}\left(\Phi^{\{n, k\}} \stackrel{(k)}{\cdot}\left(g^{T \stackrel{(k)}{\star}} \mathbf{v}^{\star}\right)\right)=g_{i_{1} j_{1}} \ldots g_{i_{n} j_{n}} \Phi_{j_{1} \ldots j_{n} k_{1} \ldots k_{k}} g_{k_{1} l_{1}}^{T} \ldots g_{k_{k} l_{k}}^{T} v_{l_{1} \ldots l_{k}}^{\star} \\
& =g_{i_{1} j_{1}} \ldots g_{i_{n} j_{n}} g_{k_{1} l_{1}}^{T} \ldots g_{k_{k} l_{k}}^{T} \Phi_{j_{1} \ldots j_{n} k_{1} \ldots k_{k}} v_{l_{1} \ldots l_{k}}^{\star} \\
& =g_{i_{1} j_{1}} \ldots g_{i_{n} j_{n}} g_{l_{1} k_{1}} \ldots g_{l_{k} k_{k}} \Phi_{j_{1} \ldots j_{n} k_{1} \ldots k_{k}} v_{l_{1} \ldots l_{k}}^{\star} \\
& =\left(g^{(n+k)} \Phi^{\{n, k\}}\right)^{(k)} \cdot \mathbf{v}^{\star} \text {. }
\end{aligned}
$$

Then

$$
\forall \mathbf{v}^{\star} \in \mathbb{K}^{k}, \forall g \in \mathrm{O}(2), \quad\left(g^{(n+k)} \Phi^{\{n, k\}}-\Phi^{\{n, k\}}\right)^{(k)} \cdot \mathbf{v}^{\star}=0
$$


which implies that

$$
\forall g \in \mathrm{O}(2), \quad g_{\star}^{(n+k)} \Phi^{\{n, k\}}=\Phi^{\{n, k\}} .
$$

Since the only isotropic tensor of odd order is the null tensor, $\Phi^{\{n, k\}}$ is null if $n$ and $k$ are of different parity. If $n$ and $k$ are of the same parity, then $\Phi^{\{n, k\}}$ is an isotropic tensor of order $n+k$, and thus can be expressed as a linear combination of elements of $\mathbb{I}^{(n+k)}$, i.e.

$$
\Phi^{\{n, k\}}=\sum_{i} \lambda_{i} \mathbf{i}_{i}^{(n+k)} .
$$

Proposition A.2. Consider $\Phi^{\{n, k\}} \in \mathbb{I}^{(n+k)}$ and $\mathbf{v} \in \mathbb{K}^{k}$. The image $\mathbf{V} \in \mathbb{H}^{(n, k)}$ of $\mathbf{v}$ by $\Phi^{\{n, k\}}$ has the following form:

$$
\mathbf{V}=\Phi^{\{n, k\}} \stackrel{(k)}{\cdot} \mathbf{v}=\sum_{j} \lambda_{j} \varsigma_{j} *\left(\mathbf{i}_{1}^{(n-k)} \otimes \mathbf{v}\right), \quad \text { with } \varsigma_{j} \in \mathfrak{S}_{n} .
$$

Proof. Since $\Phi^{\{n, k\}}=\sum_{i} \lambda_{i} \mathbf{i}_{i}^{(n+k)}, \mathbf{V}$ has the following expression

$$
\mathbf{V}=\Phi^{\{n, k\}} \stackrel{(k)}{\cdot} \mathbf{v}=\sum_{i} \lambda_{i} \mathbf{i}_{i}^{(n+k)} \stackrel{(k)}{\cdot} \mathbf{v}
$$

Since $\mathbf{v} \in \mathbb{K}^{k}, \mathbf{v}$ is totally symmetric and traceless, as such any term $\mathbf{i}_{i}^{(n+k)} \stackrel{(k)}{\cdot} \mathbf{v}$ which implies contraction within $\mathbf{v}$ disappears. The non-zero terms are, up to index permutation, those of the form $\mathbf{i}_{1}^{(n-k)} \otimes \mathbf{v}$, which gives the announced result.

Proposition A.3. Let $\mathbf{V} \in \mathbb{H}^{(n, k)}$ be the image of $\mathbf{v} \in \mathbb{K}^{k} \backslash\{0\}$ by $\Phi^{\{n, k\}} \in \mathbb{I}^{(n+k)}$. There exists $\gamma>0$ independent of $\mathbf{v}$ such that

$$
\|\mathbf{V}\|^{2}=\gamma\|\mathbf{v}\|^{2} .
$$

Proof. As

$$
\mathbf{V}=\sum_{j} \lambda_{j} \varsigma_{j} *\left(\mathbf{i}_{1}^{(n-k)} \otimes \mathbf{v}\right) \quad \text { with } \varsigma_{j} \in \mathfrak{S}_{n},
$$

we remark that $\mathbf{V}$ is null if and only if $\mathbf{v}$ is null. So let us assume that $\mathbf{v} \in \mathbb{K}^{k} \backslash\{0\}$. Since $\|\mathbf{V}\|^{2}$ and $\|\mathbf{v}\|^{2}$ are strictly positive, there exists $\gamma>0$ such $\|\mathbf{V}\|^{2}=\gamma\|\mathbf{v}\|^{2}$. Let us show that $\gamma$ is independent of $\mathbf{v}$. We consider the function $\rho: \mathbb{K}^{k} \backslash\{0\} \rightarrow \mathbb{R}^{+}$defined by

$$
\rho(\mathbf{v}):=\frac{\left\|\Phi^{\{n, k\}}{ }^{(k)} \cdot \mathbf{v}\right\|^{2}}{\|\mathbf{v}\|^{2}}
$$

in which the norms are the Frobenius norms associated with the dot product corresponding to the tensor order, i.e. $\stackrel{(k)}{.}$ for $k$ th-order tensors. Since $\mathbb{K}^{k}$ is irreducible and its elements transform as vectors, any element of $\mathbb{K}^{k}$ can be obtained from a non-null reference one, $\mathbf{v}^{1}$, up to a scaling factor and up to a rotation. We can then write

$$
\mathbf{v}=\lambda g \stackrel{(k)}{\star} \mathbf{v}^{1}, \quad \text { with } \lambda \in \mathbb{R}^{*}, \mathbf{g} \in \mathrm{O}(2),
$$

obviously the scaling and the rotation transformations commute. As a consequence, $\gamma$ is independent of $\mathbf{v}$ if the function $\rho$ is constant on $\mathbb{K}^{k} \backslash\{0\}$. Let us show that the function $\rho$ is constant on $\mathbb{K}^{k} \backslash\{0\}$.

- We observe that

$$
\forall(\lambda, \mathbf{v}) \neq(0, \mathbf{0}), \quad \rho(\lambda \mathbf{v})=\frac{\left\|\Phi^{\{n, k\}}{ }^{(k)} \cdot \lambda \mathbf{v}\right\|^{2}}{\|\lambda \mathbf{v}\|^{2}}=\frac{\left\|\Phi^{\{n, k\}}{ }^{(k)} \cdot \mathbf{v}\right\|^{2}}{\|\mathbf{v}\|^{2}}=\rho(\mathbf{v}) .
$$

So $\rho$ is an homogeneous function of degree 0 , meaning that $\rho(\mathbf{v})$ is independent of the norm of $\mathbf{v}$.

- $\rho$ is an isotropic function, i.e. $\rho(\mathbf{g} \star \mathbf{v})=\rho(\mathbf{v})$ for $\mathbf{v} \neq 0$ and $\forall \mathbf{g} \in \mathrm{O}(2)$. Firstly, $\Phi^{\{n, k\}}$ is $\mathrm{O}(2)$ equivariant, hence

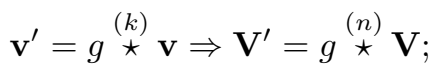


secondly, norms are isotropic functions, as such

$$
\forall \mathbf{g} \in \mathrm{O}(2), \rho(\mathbf{g} \stackrel{(k)}{\star} \mathbf{v})=\frac{\| \Phi^{\{n, k\}} \stackrel{(k)}{\cdot(\mathbf{g} \stackrel{(k)}{\star} \mathbf{v}) \|^{2}}}{\|\mathbf{g} \stackrel{(k)}{\star} \mathbf{v}\|^{2}}=\frac{\left\|\mathbf{g}^{(n)} \star \mathbf{V}\right\|^{2}}{\left\|\mathbf{g}{ }^{(k)} \mathbf{v}\right\|^{2}}=\frac{\|\mathbf{V}\|^{2}}{\|\mathbf{v}\|^{2}}=\rho(\mathbf{v}) .
$$

This result means that $\rho(\mathbf{v})$ is independent of the orientation of $\mathbf{v}$.

Since the scaling and the rotation transformations commute, and since the function is constant for both actions considered separately, we have

$$
\rho(\mathbf{v})=\rho\left(\lambda \mathbf{g} \stackrel{(k)}{\star} \mathbf{v}^{1}\right)=\rho\left(\mathbf{v}^{1}\right)=: \gamma, \quad \forall \mathbf{v} \in \mathbb{K}^{k} \backslash\{0\} .
$$

Hence the constant $\gamma$ is independent of the considered vector $\mathbf{v} \in \mathbb{K}^{k}$.

The following proposition gives a method to compute the parameter $\gamma$, it corresponds to Proposition 3.2 of Section 3.

Proposition A.4. The constant $\gamma$ defined in Proposition A.3 can be also calculated as

$$
\gamma=\frac{1}{2} \operatorname{tr} \mathrm{M}
$$

in which $\mathrm{M}$ is the matrix of the linear map $\eta: \mathbb{K}^{k} \rightarrow \mathbb{K}^{k}$ defined by

$$
\eta(\mathbf{v})=\left(\Phi^{\{k, n\}} \circ \Phi^{\{n, k\}}\right) \cdot \mathbf{v} .
$$

Proof. Let $\mathbf{v} \in \mathbb{K}^{k} \backslash\{0\}$ and $\mathbf{V}:=\Phi^{\{n, k\}} \stackrel{(k)}{\cdot} \mathbf{v}$. From Proposition A.3, there exists $\gamma>0$ such that $\|\mathbf{V}\|^{2}=\gamma\|\mathbf{v}\|^{2}$. Let us consider

$$
\mathrm{M}^{(k, k)}=\Phi^{\{k, n\}} \stackrel{(n)}{\cdot} \Phi^{\{n, k\}}
$$

which can be considered as a symmetric second-order tensor on $\mathbb{K}^{k}$. By introducing $\mathrm{I}^{\mathbb{K}^{k}}$ the second-order identity tensor on $\mathbb{K}^{k}$, the relation $\|\mathbf{V}\|^{2}=\gamma\|\mathbf{v}\|^{2}$ can be expressed as:

$$
\mathbf{v} \cdot \mathrm{M}^{(k, k)} \cdot \mathbf{v}=\gamma \mathbf{v} \cdot \mathrm{I}^{\mathbb{K}^{k}} \cdot \mathbf{v} \text {. }
$$

Differentiating with respect to $\mathbf{v}$ we obtain

$$
\left(\mathrm{M}^{(k, k)}-\gamma \mathrm{I}^{\mathbb{K}^{k}}\right) \cdot \mathbf{v}=0 .
$$

By considering a specific basis for $\mathbb{K}^{k}$, the former relation can be reformulated in terms of matrix,

$$
([\mathrm{M}]-\gamma[\mathrm{I}]) \cdot[\mathbf{v}]=0 .
$$

Since $\mathbf{v} \neq 0$ the previous relation shows that $\gamma$ is an eigenvalue of $[\mathrm{M}]$. This result can be refined by noticing the following points:

- $\forall k>0, \operatorname{dim}\left(\mathbb{K}^{k}\right)=2$, as such $[\mathrm{M}]$ has at most 2 different eigenvalues ;

- Since by construction $\mathrm{M}^{(k, k)}$ is an isotropic tensor, $[\mathrm{M}]$ is proportional to [I]. A a result, $\gamma$ is a double eigenvalue.

As a consequence,

$$
\gamma=\frac{1}{2} \operatorname{tr}[\mathrm{M}]
$$

The next result is fundamental to demonstrate Theorem 3.1.

Lemma A.5. The tensor $\Phi^{\{n, k\}}$ is invertible, and its inverse $\left(\Phi^{\{n, k\}}\right)^{-1}$ has the following expression:

$$
\left(\Phi^{\{n, k\}}\right)^{-1}=\frac{1}{\gamma} \Phi^{\{k, n\}}
$$

in which $\Phi^{\{k, n\}}$ denotes the transpose of $\Phi^{\{n, k\}}$ as defined by Equation (3.6). 
Proof. Let $\mathbf{v} \in \mathbb{K}^{k}$ and $\mathbf{V}=\Phi^{\{n, k\}}{ }^{(k)} \cdot \mathbf{v}$, we have

$$
\|\mathbf{V}\|^{2}=\left\langle\Phi^{\{n, k\}} \stackrel{(k)}{\cdot} \mathbf{v}, \Phi^{\{n, k\}} \stackrel{(k)}{\cdot} \mathbf{v}\right\rangle_{\mathbb{H}^{(n, k)}}=\left\langle\mathbf{v}, \Phi^{\{k, n\}} \stackrel{(n)}{\cdot} \Phi^{\{n, k\}} \stackrel{(k)}{\cdot} \mathbf{v}\right\rangle_{\mathbb{K}^{k}} .
$$

From Lemma A.3 we have:

$$
\|\mathbf{V}\|^{2}=\gamma\|\mathbf{v}\|^{2}
$$

then

which is equivalent to

$$
\left\langle\mathbf{v}, \Phi^{\{k, n\}} \stackrel{(n)}{\cdot} \Phi^{\{n, k\}} \stackrel{(k)}{\cdot} \mathbf{v}\right\rangle_{\mathbb{K}^{k}}=\left\langle\mathbf{v}, \gamma \mathbf{I}^{\mathbb{K}^{k}} \stackrel{(k)}{\cdot} \mathbf{v}\right\rangle_{\mathbb{K}^{k}}
$$

$$
\left\langle\mathbf{v},\left(\Phi^{\{k, n\}} \stackrel{(n)}{\cdot} \Phi^{\{n, k\}}-\gamma \mathbf{I}^{\mathbb{K}^{k}}\right) \cdot \mathbf{v}\right\rangle_{\mathbb{K}^{k}}=0 .
$$

Since this true for all $\mathbf{v} \in \mathbb{K}^{k}$, we deduce that

$$
\Phi^{\{k, n\}} \stackrel{(n)}{\cdot\{n, k\}}=\gamma \mathbf{I}^{\mathbb{K}^{k}}
$$

and then

$$
\left(\Phi^{\{n, k\}}\right)^{-1}=\frac{1}{\gamma} \Phi^{\{k, n\}}
$$

Finally, the next result will be used in Appendix B to directly characterize some harmonic embeddings.

Lemma A.6. Consider $\mathbb{T}^{n}$ a space of $n$ th-order tensors and let $\mathbb{H}^{(n, k)}$ be a subspace of $\mathbb{T}^{n}$ isomorphic to a harmonic space $\mathbb{K}^{k}$ with $k \leq n$. Then the projector $\mathrm{P}^{(n, k)}$ from $\mathbb{T}^{n}$ onto $\mathbb{H}{ }^{(n, k)}$ belongs to $\mathcal{L}^{s}\left(\mathbb{T}^{n}, \mathbb{T}^{n}\right)$.

Proof. By definition, $\mathrm{P}^{(n, k)}$ is a linear map from $\mathbb{T}^{n}$ to $\mathbb{T}^{n}$. We can therefore consider it as a tensor of order $2 n$. It remains to verify the major symmetry, i.e. that $\mathrm{P}^{(n, k)}=\left(\mathrm{P}^{(n, k)}\right)^{T}$. Since

$$
\mathrm{P}^{(n, k)}=\frac{1}{\gamma} \Phi^{\{n, k\}}{ }^{(k)}\left(\Phi^{\{n, k\}}\right)^{T},
$$

the major symmetry is verified.

\section{Appendix B. Clebsch-Gordan harmonic embeddings}

This appendix is devoted to the demonstrations of the fundamental explicit harmonic decompositions associated with the embeddings $\mathbb{K}^{p+q} \oplus \mathbb{K}^{|p-q|} \hookrightarrow \mathbb{K}^{p} \otimes \mathbb{K}^{q}$ used in the main part of the article. Since the embedding of $\mathbb{K}^{p+q}$ into $\mathbb{K}^{p} \otimes \mathbb{K}^{q}$ is trivial, the problem reduces to the determination of the unique embedding of $\mathbb{K}^{|p-q|}$ into $\mathbb{K}^{p} \otimes \mathbb{K}^{q}$. Knowing the algebraic characterisation of $\mathbb{K}^{p} \otimes \mathbb{K}^{q}$, this question can be reformulated in terms of linear algebra.

We have the following result:

Theorem B.1. For $n \geq 1$, let $\mathbf{P}^{(n, n)}$ be the tensor associated to the projector from $\mathbb{T}^{n}$ onto $\mathbb{K}^{n}$ and consider $\mathbf{T}^{n, n}$ an element of $\mathcal{L}^{s}\left(\mathbb{K}^{n}, \mathbb{K}^{n}\right) \simeq \mathbb{K}^{n} \otimes^{s} \mathbb{K}^{n}$. The tensor $\mathbf{T}^{n, n}$ can be parametrized as follows:

$$
\mathbf{T}^{n, n}=\mathbf{K}+\frac{\alpha}{2} \mathbf{P}^{(n, n)}, \quad(\mathbf{K}, \alpha) \in \mathbb{K}^{2 n} \times \mathbb{K}^{0},
$$

in such a way that $\mathbf{T}^{n, n}{ }^{(2 n)} \mathbf{P}^{(n, n)}=\alpha$.

Proof. First, by using the Clebsch-Gordan formula, it is known that $\mathcal{L}^{s}\left(\mathbb{K}^{n}, \mathbb{K}^{n}\right) \simeq \mathbb{K}^{2 n} \oplus \mathbb{K}^{0}$. As a consequence $\mathbf{T}^{n, n} \in \mathcal{L}^{s}\left(\mathbb{K}^{n}, \mathbb{K}^{n}\right)$ can be written as

$$
\mathbf{T}^{n, n}=\mathbf{K}+\alpha \Phi^{\{2 n, 0\}}
$$

with $\mathbf{K} \in \mathbb{K}^{2 n}, \alpha \in \mathbb{K}^{0}$ and $\Phi^{\{2 n, 0\}}$ is an isotropic tensor of order $2 n$ element of $\mathcal{L}^{s}\left(\mathbb{T}^{n}, \mathbb{T}^{n}\right)$. As a direct consequence of Lemma A.6, it can be observed that $\mathbf{P}^{(n, n)}$ is also an isotropic tensor of order $2 n$ element of $\mathcal{L}^{s}\left(\mathbb{T}^{n}, \mathbb{T}^{n}\right)$. Since $\operatorname{dim}\left(\mathbb{H}^{(n, 0)}\right)=1$,

$$
\Phi^{\{2 n, 0\}}=\lambda \mathbf{P}^{(n, n)} .
$$

The scaling factor $\lambda$ is determined so that $\mathbf{T}^{n, n}{ }^{(2 n)} \mathbf{P}^{(n, n)}=\alpha$. We have

$$
\mathbf{T}^{n, n} \stackrel{(2 n)}{\cdot} \mathbf{P}^{(n, n)}=\alpha \lambda \mathbf{P}^{(n, n)} \stackrel{(2 n)}{\cdot} \mathbf{P}^{(n, n)} .
$$


Since $\mathbf{P}^{(n, n)}=\mathrm{I}^{\mathbb{K}^{n}},\left(\mathbf{P}^{(n, n)} \stackrel{(2 n)}{\cdot} \mathbf{P}^{(n, n)}\right)=\operatorname{dim}\left(\mathbb{K}^{n}\right)=2$. Then $\mathbf{T}^{n, n} \stackrel{(2 n)}{{ }^{(n, n)}}=2 \alpha \lambda$ and we deduce that $\lambda=\frac{1}{2}$.

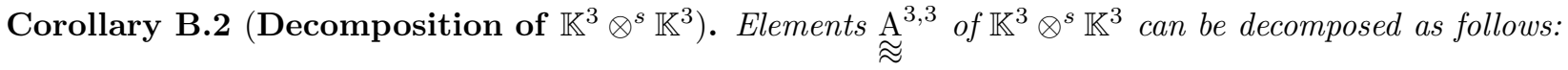

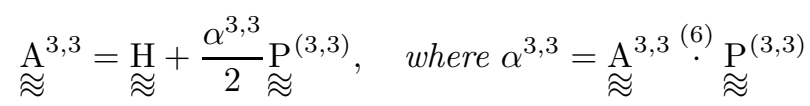

with $\underset{\approx}{\mathrm{P}^{(3,3)}}$ defined in Proposition 5.4.

Lemma B.3 (Decomposition of $\left.\mathbb{K}^{3} \otimes \mathbb{K}^{1}\right)$. There exists an $\mathrm{O}(2)$-equivariant isomorphism between $\mathbb{K}^{3} \otimes \mathbb{K}^{1}$

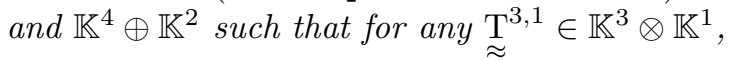

$$
\underset{\approx}{\mathrm{T}^{3,1}}=\underset{\approx}{\mathrm{H}}+\underset{\approx}{\Phi} \underset{\sim}{\{4,2\}}: \mathrm{h}
$$

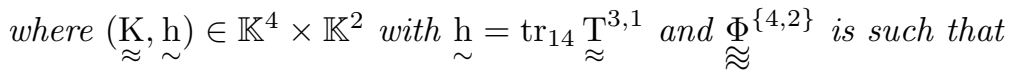

$$
\left({\underset{\Phi}{\Phi}}^{\{4,2\}}: \underset{\sim}{\mathrm{h}}\right)_{i j k l}=\frac{1}{2}\left(h_{i j} \delta_{k l}+h_{i k} \delta_{j l}-h_{i l} \delta_{j k}\right)
$$

and

$$
\left({\underset{\Phi}{\Phi}}^{\{4,2\}}\right)_{i j k l m n}=\frac{1}{2}\left(\delta_{k l} P_{i j m n}^{(2,2)}+\delta_{j l} P_{i k m n}^{(2,2)}-\delta_{j k} P_{i l m n}^{(2,2)}\right) .
$$

Above, $\underset{\approx}{\mathrm{P}^{(2,2)}}$ is the standard deviatoric projector defined in Equation (4.5). Moreover, the inverse of $\underset{\approx}{\approx 4,2\}}$ is given by

$$
\left({\underset{\Pi}{\approx}}^{\{2,4\}}\right)_{i j k l m n}={\underset{\approx}{\Phi_{k l m n i j}}}_{\underset{\approx}{2}, 4\}}=\frac{1}{2}\left(\delta_{m n} P_{k l i j}^{(2,2)}+\delta_{l n} P_{k m i j}^{(2,2)}-\delta_{l m} P_{k n i j}^{(2,2)}\right) .
$$

Proof. From the Clebsch-Gordan formula in 2D, it is known that

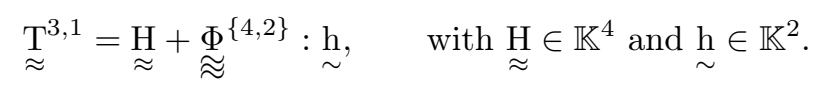

It can be checked that $\mathbb{K}^{4} \subset \mathbb{K}^{3} \otimes \mathbb{K}^{1}$ and the question is the embedding of $\mathbb{K}^{2}$ into $\mathbb{K}^{3} \otimes \mathbb{K}^{1}$. Up to a scaling factor, there is a unique way to do so. The embedding can be determined by solving a linear system. A general embedding of $\mathbb{K}^{2}$ into $\stackrel{4}{\otimes} \mathbb{R}^{2}$ is given by:

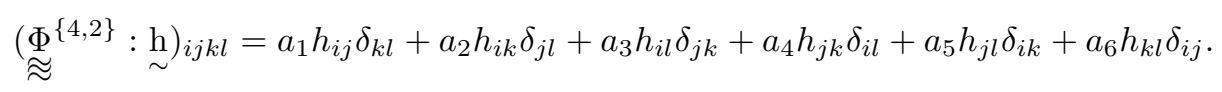

It can be checked that, in $\mathbb{R}^{2}$, for a generic $\underset{\sim}{\mathrm{h}} \in \mathbb{K}^{2}$, the family of tensors $\left\{h_{i j} \delta_{k l}, h_{i k} \delta_{j l}, h_{i l} \delta_{j k}, h_{j k} \delta_{i l}, h_{j l} \delta_{i k}, h_{k l} \delta_{i j}\right\}$ is not free. For instance,

$$
\left\{\begin{array}{l}
h_{k l} \delta_{i j}=h_{i l} \delta_{j k}+h_{j k} \delta_{i l}-h_{i j} \delta_{k l} \\
h_{j l} \delta_{i k}=h_{i l} \delta_{j k}+h_{j k} \delta_{i l}-h_{i k} \delta_{j l} .
\end{array}\right.
$$

However, the same family restricted to its four first elements is free. As a consequence, we will consider the following free parametrization:

$$
\left(\underset{\Phi}{\stackrel{\Phi}{\approx}}{ }^{4,2\}}: \underset{\sim}{h}\right)_{i j k l}=b_{1} h_{i j} \delta_{k l}+b_{2} h_{i k} \delta_{j l}+b_{3} h_{i l} \delta_{j k}+b_{4} h_{j k} \delta_{i l} .
$$

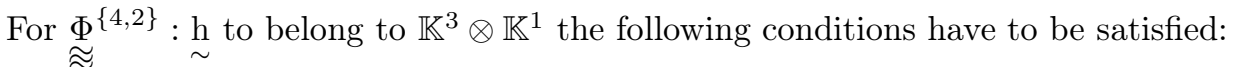

(1) complete symmetry with respect to $(i j k)$ :

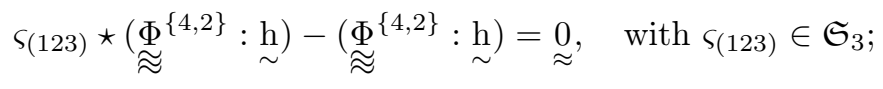

(2) traceless with respect to $(i j k)$ :

$$
\stackrel{\mathrm{i}}{\sim}^{(2)}:\left(\underset{\approx}{\Phi^{\{4,2\}}: \mathrm{h}}\right)=\underset{\sim}{\sim} .
$$




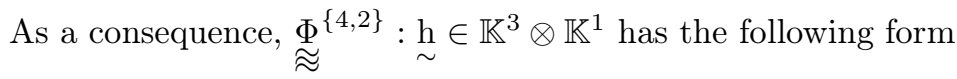

$$
\left(\stackrel{\Phi}{\Phi}^{\{4,2\}}: \underset{\sim}{\mathrm{h}}\right)_{i j k l}=b_{1}\left(h_{i j} \delta_{k l}+h_{i k} \delta_{j l}-h_{i l} \delta_{j k}\right) .
$$

The value of $b_{1}$ is determined by the closure condition:

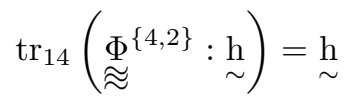

which implies that $b_{1}=\frac{1}{2}$. So, at the end:

$$
\left({\underset{\approx}{\Phi}}^{\{4,2\}}: \underset{\sim}{\mathrm{h}}\right)_{i j k l}=\frac{1}{2}\left(h_{i j} \delta_{k l}+h_{i k} \delta_{j l}-h_{i l} \delta_{j k}\right)
$$

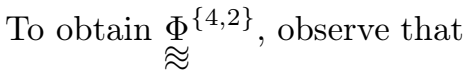

$$
\underset{\sim}{\mathrm{h}}=\underset{\approx}{\mathrm{I}^{\mathbb{K}^{2}}}: \underset{\sim}{\mathrm{h}}=\underset{\approx}{\mathrm{P}} \mathrm{P}^{(2,2)}: \underset{\sim}{\mathrm{h}} .
$$

Inserting this relation into Equation (B.1), we obtain the expression of $\Phi^{(4,2)}$. Since $\left\|\underset{\approx}{\Phi^{\{4,2\}}: \mathrm{h}}\right\|^{2}=\|\underset{\approx}{\mathrm{h}}\|^{2}$, the application of Theorem 3.1 gives

$$
{\underset{\approx}{\approx}}^{\{2,4\}}=\left({\underset{\approx}{\Phi}}^{\{4,2\}}\right)^{T}
$$

and a direct computation allows us to check that

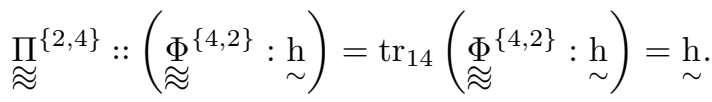

The structure of the harmonic embedding is summed-up in the following diagram

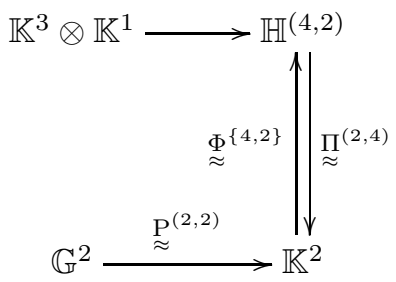

For the three next lemmas, the proofs follow the same lines and will not be detailed.

Lemma B.4 (Decomposition of $\mathbb{K}^{2} \otimes \mathbb{K}^{3}$ ). There exists an $\mathrm{O}(2)$-equivariant isomorphism between $\mathbb{K}^{2} \otimes \mathbb{K}^{3}$

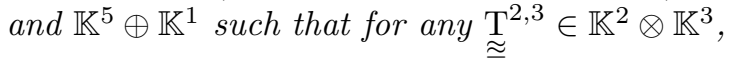

$$
\stackrel{\mathrm{T}}{\overparen{2}}^{2,3}=\underset{\approx}{\mathrm{H}}+{\underset{\Phi}{\Phi}}^{\{5,1\}} \cdot \underline{\mathrm{v}}
$$

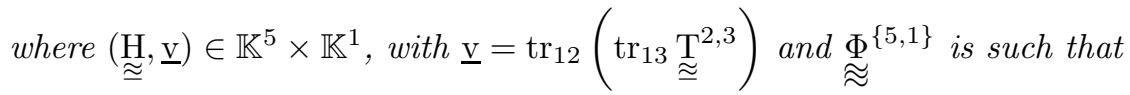

$$
\begin{aligned}
\left(\stackrel{\Phi}{\Phi}^{\{5,1\}} \cdot \underline{\mathrm{v}}\right)_{i j k l m} & =\frac{1}{4}\left(v_{i}\left(\delta_{j k} \delta_{l m}-\delta_{j l} \delta_{k m}-\delta_{j m} \delta_{k l}\right)-v_{j} \delta_{i m} \delta_{k l}\right. \\
& \left.+v_{k}\left(-2 \delta_{i j} \delta_{l m}+\delta_{i l} \delta_{j m}+2 \delta_{i m} \delta_{j l}\right)+v_{l} \delta_{i k} \delta_{j m}+v_{m} \delta_{i j} \delta_{k l}\right),
\end{aligned}
$$

Intrinsically ,

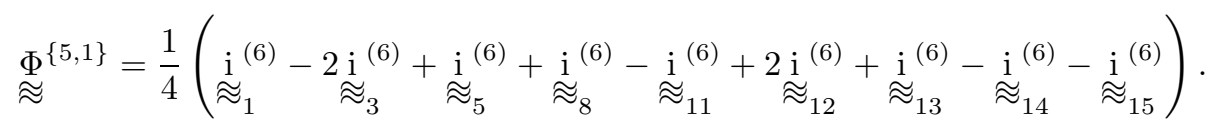

The inverse of $\underset{\approx}{\Phi^{\{5,1\}}}$ is given by

$$
\left(\prod^{\{1,5\}}\right)_{i j k l m n}=\left({\underset{\approx}{(5,1\}}}^{\{k k l m n i} .\right.
$$


Lemma B.5 (Decomposition of $\left.\mathbb{K}^{2} \otimes \mathbb{K}^{1}\right)$. There exists an $\mathrm{O}(2)$-equivariant isomorphism between $\mathbb{K}^{2} \otimes \mathbb{K}^{1}$ and $\mathbb{K}^{3} \oplus \mathbb{K}^{1}$ such that

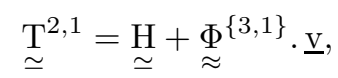

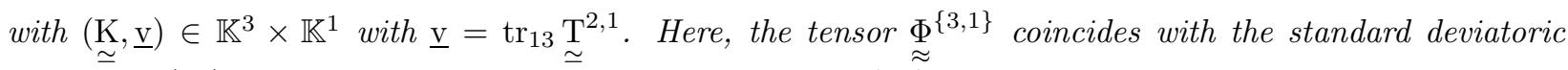

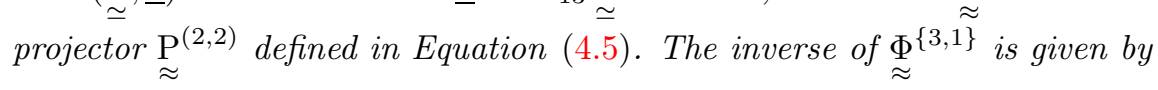

$$
\left({\underset{\approx}{\Pi}}^{\{1,3\}}\right)_{i j k l}=\left({\underset{\mathrm{P}}{(2,2)}}^{(2 i j k}\right. \text {. }
$$

Lemma B.6. There exists an $\mathrm{O}(2)$-equivariant embedding of $\mathbb{K}^{-1}$ into $\mathbb{G}^{2}$ such that

$$
\stackrel{\mathrm{T}}{ }^{1,1}=\beta \underset{\sim}{\Phi^{\{2,-1\}}}
$$

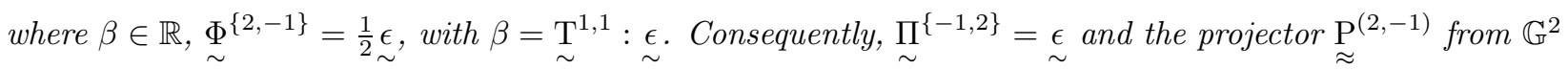
onto $\mathbb{H}^{(2,-1)}$ has the following expression:

$$
{\underset{\mathrm{P}}{(2,-1)}}^{\left(2, \frac{1}{2} \epsilon\right.} \otimes \underset{\sim}{\epsilon}
$$

\section{Appendix C. Alternative decomposition of $\mathbb{T}_{(i j) k}$}

Due to the multiplicity of $\mathbb{K}^{1}$ in the harmonic structure of $\mathbb{T}_{(i j) k}$, its explicit harmonic decomposition is not uniquely defined [38]. As a consequence of the Clebsch-Gordan Harmonic Algorithm, considering another explicit decomposition for the state tensor space will affect the explicit harmonic decomposition of the associated constitutive tensors. In this appendix we propose another decomposition for the tensors in $\mathbb{T}_{(i j) k}$ which is used in strain-gradient elasticity [29] and in strain-gradient plasticity [67, 43]. In the modified strain-gradient theory developed in [29], the strain-gradient tensor is reduced to the gradient of the hydrostatic strain tensor. In contrast, in the incompressible strain-gradient plasticity theory [67, 43], this contribution is constrained to be zero. In both theories, the constraint relates to a derivative of the strain tensor. Consequently, we introduce here a decomposition of $\mathbb{T}_{(i j) k}$ based on the derivation of the harmonic decomposition of symmetric second-order tensors. This approach, which is related to Mindlin Type II formulation, can be described as follows :

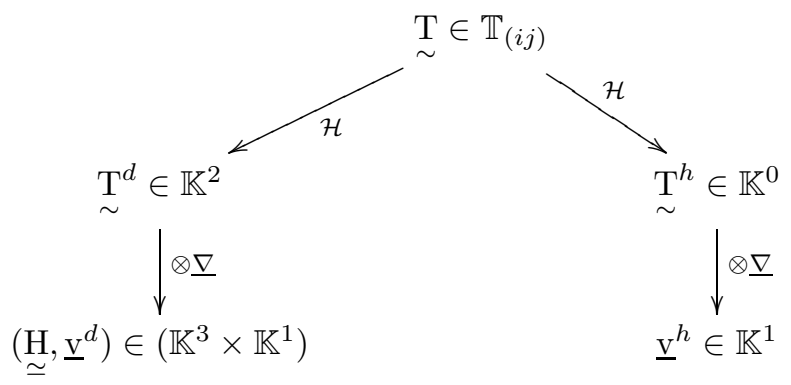

Associated to this decomposition we have the following result:

Theorem C.1 (Alternative Harmonic Decomposition of $\left.\mathbb{T}_{(i j) k}\right)$. There exists an $\mathrm{O}(2)$-equivariant isomorphism between $\mathbb{T}_{(i j) k}$ and $\mathbb{K}^{3} \oplus \mathbb{K}^{1} \oplus \mathbb{K}^{1}$ such that for $\underset{\simeq}{\mathrm{H}} \in \mathbb{K}^{3}$ and $\left(\underline{\mathrm{v}}^{d}, \underline{\mathrm{v}}^{h}\right) \in \mathbb{K}^{1} \times \mathbb{K}^{1}$,

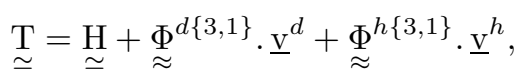

with $\left({\underset{\Phi}{\Phi}}^{d\{3,1\}},{\underset{\Phi}{\Phi}}^{h\{3,1\}}\right)$ the harmonic embeddings of the form:

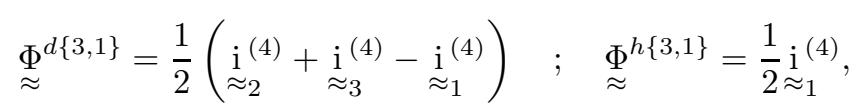

in which $\left(\underset{\approx}{\mathrm{i}}(4), \underset{\approx}{\mathrm{i}}(4), \underset{\approx}{\mathrm{i}^{(4)}}\right)$ are the fourth-order elementary isotropic tensors defined by Equation (1.1). Conversely, for any $\left.\underset{\simeq}{\mathrm{T}} \in \mathbb{T}_{(i j) k}, \underset{\simeq}{\mathrm{H}}, \underline{\mathrm{v}}^{d}, \underline{\mathrm{v}}^{h}\right) \in \mathbb{K}^{3} \times \mathbb{K}^{1} \times \mathbb{K}^{1}$ are defined from $\underset{\simeq}{\mathrm{T}}$ as follows: 


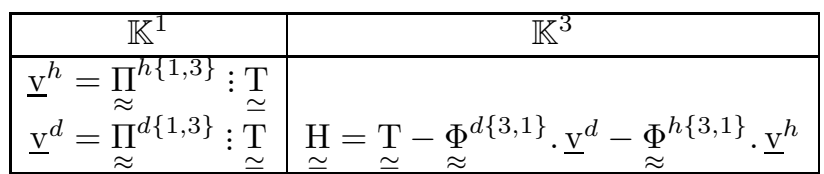

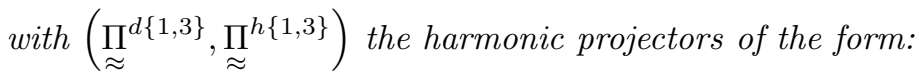

$$
\prod_{\approx} \prod^{d\{1,3\}}=\frac{1}{2}\left(\underset{\approx_{2}^{(4)}}{\underset{\mathrm{i}_{2}}{\mathrm{i}}}{\underset{\approx}{(4)}}^{(4)} \underset{\approx_{3}^{(4)}}{\mathrm{i}}\right) \quad ; \quad \underset{\approx}{\prod^{h\{1,3\}}}=\underset{\approx_{3}^{\mathrm{i}}}{(4)} .
$$

From the embedding operators involved in Theorem 3.1 a family of projectors can be deduced.

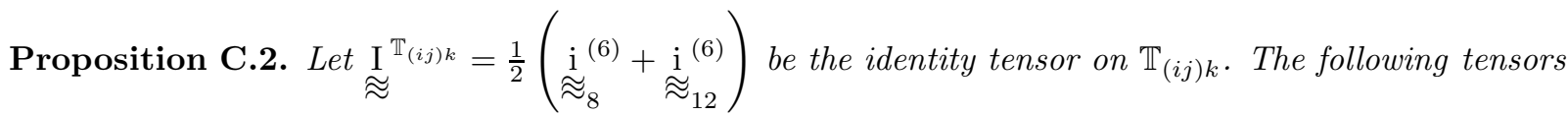

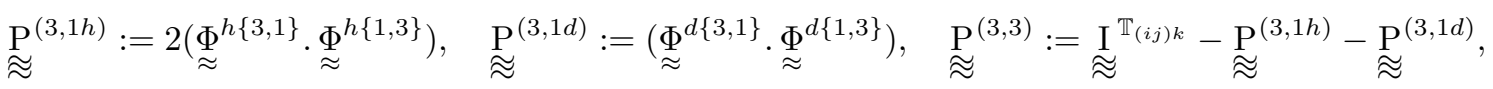

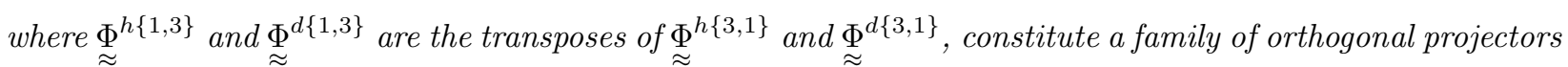
on $\underset{\mathbb{H}^{h(3,1)}}{\approx}, \mathbb{H}^{d(3,1)}$ and $\mathbb{K}^{3}$, respectively.

In this Proposition, the spaces $\mathbb{H}^{d(3,1)}$ and $\mathbb{H}^{h(3,1)}$ are defined as follows. $\mathbb{H}^{d(3,1)}$ is the subspace of $\mathbb{K}^{2} \otimes \mathbb{K}^{1}$ of tensors orthogonal to elements in $\mathbb{K}^{3}$ :

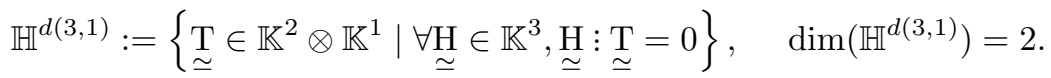

Unlike the tensors belonging to $\mathbb{K}^{3}$, the elements of $\mathbb{H}^{d(3,1)}$ are traceless only with respect to their first two indices. The space $\mathbb{H}^{h(3,1)}$ is defined as

$$
\mathbb{H}^{h(3,1)}:=\left\{\underset{\simeq}{\mathrm{T}} \in \mathbb{T}_{(i j) k} \mid \underset{\simeq}{\forall \mathrm{H}} \in \mathbb{T}_{(i j) k}, \underset{\simeq}{\mathrm{H}}: \underset{\simeq}{\mathrm{T}}=0\right\}, \quad \operatorname{dim}\left(\mathbb{H}^{h(3,1)}\right)=2 .
$$

It is the subspace of $\mathbb{T}_{(i j) k}$ of tensors having none vanishing trace (12).

Proposition C.3. The tensor $\underset{\approx}{\mathrm{M}} \in \mathbb{E} \mathrm{la}_{5}$ admits the uniquely defined Intermediate Block Decomposition associated to the family of projectors $\left.\underset{\approx}{\left(\mathrm{P}^{(3,3)}\right.}, \underset{\approx}{\mathrm{P}^{(3,1 d)}}, \underset{\approx}{\mathrm{P}^{(3,1 h)}}, \underset{\approx}{\mathrm{P}^{(2,2)}}, \underset{\approx}{\mathrm{P}^{(2,0)}}\right)$ :

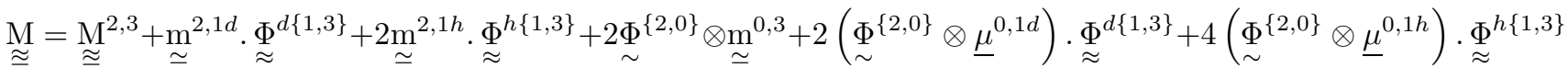

\begin{tabular}{|c|c|c|}
\hline $\mathbb{T}^{1}$ & $\mathbb{T}^{3}$ & $\mathbb{T}^{5}$ \\
\hline$\underline{\mu}^{0,1 d}:={\underset{\sim}{\Phi}}^{\{0,2\}}: \underset{\approx}{\mathrm{M}}: \underset{\approx}{\Phi^{d\{3,1\}}}$ & ${\underset{\simeq}{\simeq}}^{2,1 d}:=\underset{\approx}{\mathrm{P}^{(2,2)}}: \underset{\approx}{\mathrm{M}}:{\underset{\approx}{\Phi}}^{d\{3,1\}}$ & $\stackrel{\mathrm{M}}{\approx}^{2,3}:=\underset{\approx}{\mathrm{P}^{(2,2)}}: \underset{\approx}{\mathrm{M}}:{\underset{\approx}{\mathrm{P}}}^{(3,3)}$ \\
\hline$\underline{\mu}^{0,1 h}:={\underset{\sim}{\Phi}}^{\{0,2\}}: \underset{\approx}{\mathrm{M}}:{\underset{\approx}{\Phi}}^{h\{3,1\}}$ & 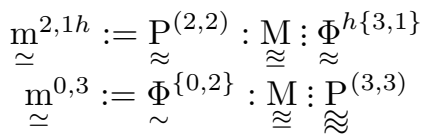 & \\
\hline
\end{tabular}
in which $\underset{\approx}{\mathrm{M}}{ }^{2,3} \in \mathbb{K}^{2} \otimes \mathbb{K}^{3},\left(\underset{\simeq}{\mathrm{m}^{2,1 d}}, \underset{\simeq}{\mathrm{m}^{2,1 h}}\right) \in\left(\mathbb{K}^{2} \otimes \mathbb{K}^{1}\right)^{2},{\underset{\sim}{\mathrm{m}}}^{0,3} \in \mathbb{K}^{3},\left(\underline{\mu}^{0,1 d}, \underline{\mu}^{0,1 h}\right) \in\left(\mathbb{K}^{1}\right)^{2}$, and $\underset{\approx}{\Phi^{d\{3,1\}}}, \underset{\approx}{\Phi^{h\{3,1\}}}$ and $\underset{\sim}{\Phi^{\{0,2\}}}$ are defined, respectively, in Propositions 5.1 and 4.2. Those elements are defined from $\underset{\approx}{\mathrm{M}}$ as follows:

Proposition C.4 (Clebsch-Gordan Harmonic Decomposition of $\underset{\approx}{\mathrm{M}} \in \mathbb{E} \mathrm{la}_{5}$ ). The tensor $\underset{\approx}{\mathrm{M}} \in \mathbb{E} \mathrm{la}_{5}$ admits the uniquely defined Clebsch-Gordan Harmonic Decomposition associated to the family of projectors

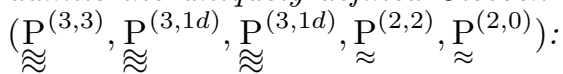

$$
\begin{aligned}
& \underset{\approx}{\mathrm{M}}=\underset{\approx}{\mathrm{H}^{2,3}}+\underset{\simeq}{\mathrm{H}^{2,1 d}} \cdot \underset{\approx}{\Phi} \Phi^{d 1,3\}}+\underset{\simeq}{\mathrm{H}^{2,1 h}} \cdot \underset{\approx}{\Phi^{h\{1,3\}}}+2 \underset{\sim}{\Phi^{\{2,0\}}} \otimes \underset{\simeq}{\mathrm{H}^{0,3}}+\underset{\approx}{\Phi^{\{5,1\}}} \cdot \underline{\mathrm{v}}^{2,3}
\end{aligned}
$$

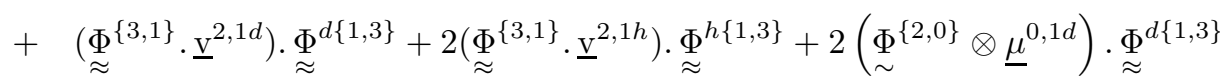

$$
\begin{aligned}
& +4\left({\underset{\sim}{\Phi}}^{\{2,0\}} \otimes \underline{\mu}^{0,1 h}\right) \cdot \Phi^{\Phi^{h\{1,3\}}}
\end{aligned}
$$


in which $\left.\underset{\approx}{\mathrm{H}^{2,3}} \in \mathbb{K}^{5}, \underset{\simeq}{\left(\mathrm{H}^{2,1 d}\right.},{\underset{\sim}{\mathrm{H}}}^{2,1 h}, \underline{\simeq}^{0,3}\right) \in\left(\mathbb{K}^{3}\right)^{3},\left(\underline{\mathrm{v}}^{2,3}, \underline{\mathrm{v}}^{2,1 d}, \underline{\mathrm{v}}^{2,1 h}, \underline{\mathrm{v}}^{0,1 d}, \underline{\mathrm{v}}^{0,1 h}\right) \in\left(\mathbb{K}^{1}\right)^{5}$. Those elements are defined from $\underset{\approx}{\mathrm{M}}$ as follows:

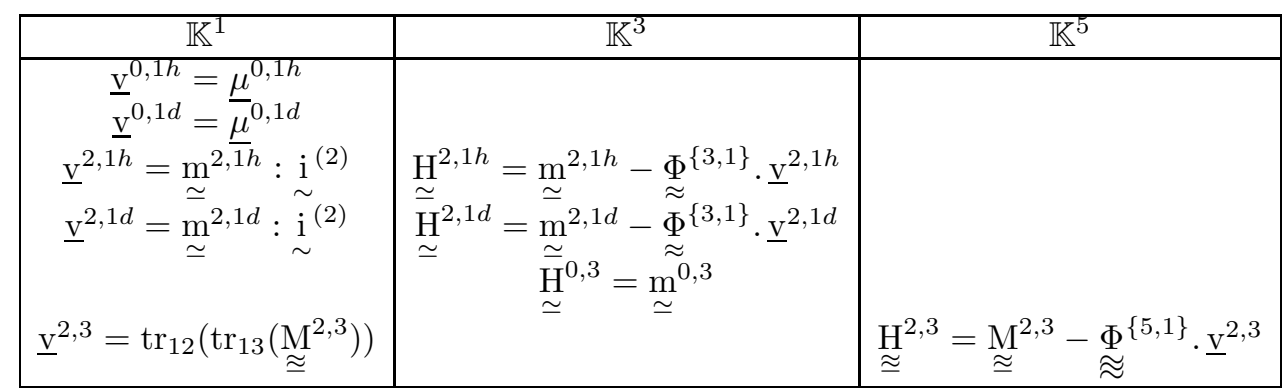

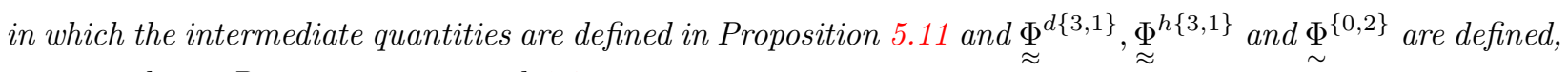
respectively, in Propositions 5.1 and 4.2.

Proposition C.5. The tensor $\underset{\approx}{\mathrm{A}} \in \mathbb{E} \mathrm{la}_{6}$ admits the uniquely defined Intermediate Block Decomposition associated to the family of projectors $\left.\underset{\approx}{\stackrel{\left(\mathrm{P}^{(3,3)}\right.}{\approx}} \underset{\mathrm{P}}{(3,1 d)}, \underset{\mathrm{P}}{(3,1 h)}\right)$ :

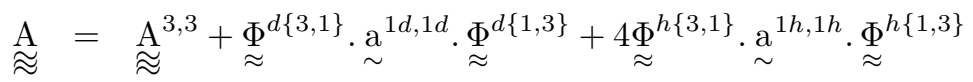

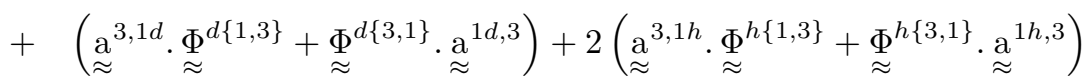

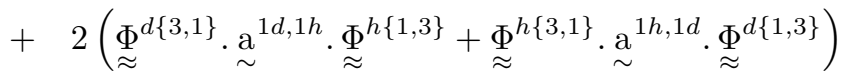

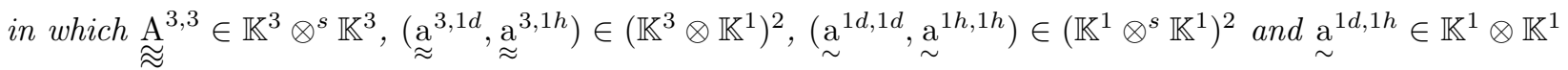

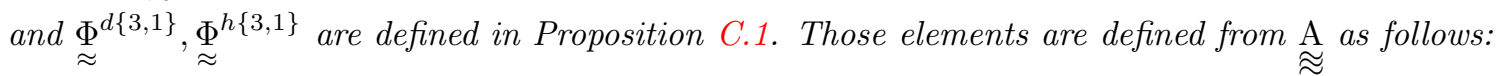

\begin{tabular}{|c|c|c|}
\hline $\mathbb{T}^{2}$ & $\mathbb{T}^{4}$ & $\mathbb{T}^{6}$ \\
\hline 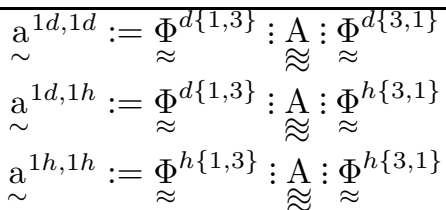 & 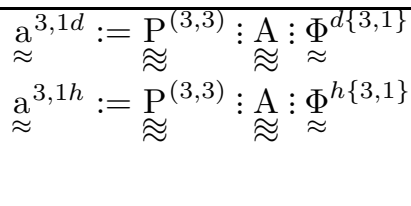 & 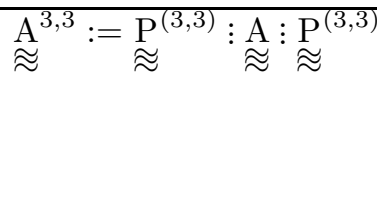 \\
\hline
\end{tabular}

Proposition C.6 (Clebsch-Gordan Harmonic Decomposition of $\underset{\approx}{\mathrm{A}} \in \mathbb{E} \mathrm{la}_{6}$ ). The tensor $\underset{\approx}{\mathrm{A}} \in \mathbb{E} \mathrm{la}_{6}$ admits the uniquely defined Clebsch-Gordan Harmonic Decomposition associated to the family of projectors 


$$
\begin{aligned}
& \left(\mathrm{P}^{(3,3)}, \underset{\mathrm{P}^{(3,1 d)}}{\approx} \underset{\mathrm{P}}{\left.\mathrm{P}^{(3,1 h)}\right)}\right.
\end{aligned}
$$

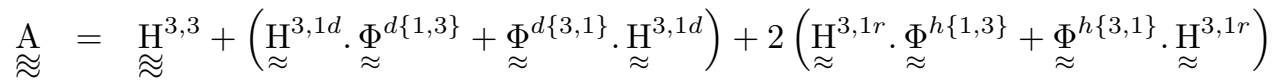

$$
\begin{aligned}
& +\underset{\approx}{\Phi^{d\{3,1\}}} \cdot{\underset{\sim}{h^{1 d, 1 d}}}^{\underset{\approx}{\Phi}}{ }^{d\{1,3\}}+4 \underset{\approx}{\Phi^{h\{3,1\}}} \cdot \operatorname{\sim }^{1 r, 1 r} \cdot{\underset{\approx}{\Phi}}^{h\{1,3\}}
\end{aligned}
$$

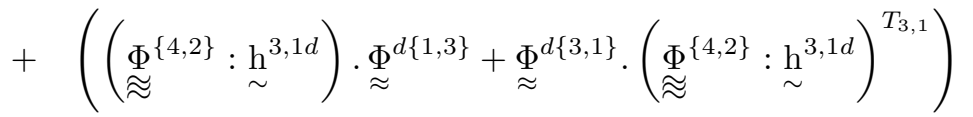

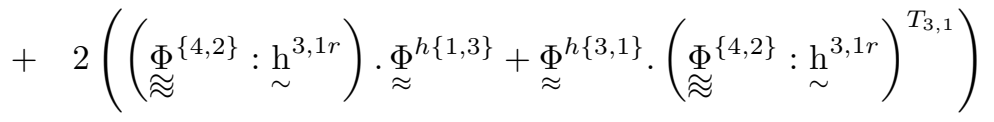

$$
\begin{aligned}
& +2\left(\underset{\approx}{\Phi^{d\{3,1\}}} \cdot \sim_{\sim}^{1 d, 1 r} \cdot \underset{\approx}{\Phi^{h\{1,3\}}}+\underset{\approx}{\Phi^{h\{3,1\}}} \cdot \mathrm{h}^{1 r, 1 d} \cdot \underset{\approx}{\Phi^{d\{1,3\}}}\right)
\end{aligned}
$$

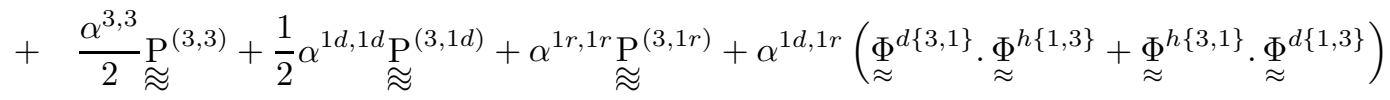

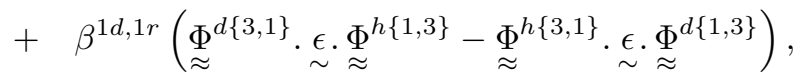

in which $\left.\left.\underset{\approx}{\mathrm{H}^{3,3}} \in \mathbb{K}^{6}, \underset{\approx}{\mathrm{H}^{3,1 d}}, \underset{\approx}{\mathrm{H}^{3,1 r}}\right) \in\left(\mathbb{K}^{4}\right)^{2}, \underset{\sim}{\mathrm{h}^{3,1 d}}, \underset{\sim}{\mathrm{h}^{3,1 r}}, \underset{\sim}{\mathrm{h}^{1 d, 1 r}}, \underset{\sim}{\mathrm{h}^{1 d, 1 d}} \underset{\sim}{\mathrm{h}^{1 r, 1 r}}\right) \in\left(\mathbb{K}^{2}\right)^{5},\left(\alpha^{3,3}, \alpha^{1 d, 1 d}, \alpha^{1 r, 1 r}, \alpha^{1 r, 1 d}\right) \in$ $\left(\mathbb{K}^{0}\right)^{4}$ and $\beta^{1 r, 1 d} \in \mathbb{K}^{-1}$. Those elements are defined from $\mathrm{A}$ as follows:

\begin{tabular}{|c|c|c|c|c|}
\hline $\mathbb{K}^{-1}$ & $\mathbb{K}^{0}$ & $\mathbb{K}^{2}$ & $\mathbb{K}^{4}$ & $\mathbb{K}^{6}$ \\
\hline$\beta^{1 d, 1 h}={\underset{\sim}{\mathrm{a}}}^{1 d, 1 h}: \underset{\sim}{\epsilon}$ & 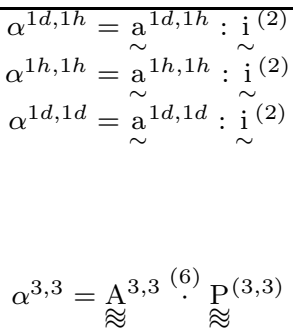 & 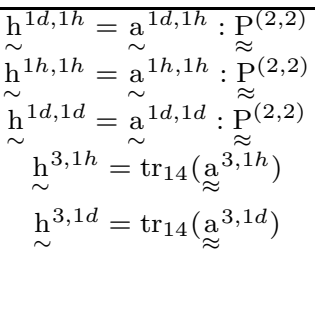 & 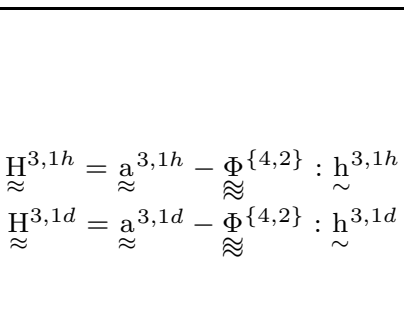 & ${\underset{\approx}{\mathrm{H}}}^{3,3}=\underset{\approx}{\mathrm{A}^{3,3}}-\frac{\alpha^{3,3}}{2} \underset{\approx}{\mathrm{*}}{ }^{(3,3)}$ \\
\hline
\end{tabular}

in which the intermediate quantities are defined in Proposition C.5. 


\section{REFERENCES}

[1] H. Abdoul-Anziz and P. Seppecher. Strain gradient and generalized continua obtained by homogenizing frame lattices. Mathematics and Mechanics of Complex Systems, 6(3):213-250, 2018.

[2] D. Abueidda, M. Bakir, R. Al-Rub, J. Bergström, N. Sobh, and I. Jasiuk. Mechanical properties of 3D printed polymeric cellular materials with triply periodic minimal surface architectures. Materials $\&$ Design, 122:255-267, 2017.

[3] J.-J. Alibert, P. Seppecher, and F. dell'Isola. Truss modular beams with deformation energy depending on higher displacement gradients. Mathematics and Mechanics of Solids, 8(1):51-73, 2003.

[4] G. Allaire, P. Geoffroy-Donders, and O. Pantz. Topology optimization of modulated and oriented periodic microstructures by the homogenization method. Computers 83 Mathematics with Applications, 78(7):2197-2229, 2019.

[5] J. Altenbach, H. Altenbach, and V. Eremeyev. On generalized Cosserat-type theories of plates and shells: a short review and bibliography. Archive of Applied Mechanics, 80(1):73-92, 2010.

[6] H. Askes and E. C. Aifantis. Gradient elasticity in statics and dynamics: an overview of formulations, length scale identification procedures, finite element implementations and new results. International Journal of Solids and Structures, 48(13):1962-1990, 2011.

[7] N. Auffray, R. Bouchet, and Y. Bréchet. Derivation of anisotropic matrix for bi-dimensional strain-gradient elasticity behavior. International Journal of Solids and Structures, 46(2):440-454, 2009.

[8] N. Auffray, J. Dirrenberger, and G. Rosi. A complete description of bi-dimensional anisotropic strain-gradient elasticity. International Journal of Solids and Structures, 69-70:195-206, 2015.

[9] N. Auffray, B. Kolev, and M. Olive. Handbook of bi-dimensional tensors: Part I: Harmonic decomposition and symmetry classes. Mathematics and Mechanics of Solids, 22(9):1847-1865, 2017.

[10] N. Auffray and P. Ropars. Invariant-based reconstruction of bidimensionnal elasticity tensors. International Journal of Solids and Structures, 87:183-193, 2015.

[11] A. Bacigalupo and L. Gambarotta. Second-order computational homogenization of heterogeneous materials with periodic microstructure. ZAMM-Journal of Applied Mathematics and Mechanics/Zeitschrift für Angewandte Mathematik und Mechanik, 90(10-11):796-811, 2010.

[12] A. Bacigalupo and L. Gambarotta. Second-gradient homogenized model for wave propagation in heterogeneous periodic media. International Journal of Solids and Structures, 51(5):1052-1065, 2014.

[13] E. Barchiesi, F. dell'Isola, F. Hild, and P. Seppecher. Two-dimensional continua capable of large elastic extension in two independent directions: asymptotic homogenization, numerical simulations and experimental evidence. Mechanics Research Communications, 103:103466, 2020.

[14] M. Bendsoe and O. Sigmund. Topology optimization: theory, methods, and applications. Springer Science \& Business Media, 2013.

[15] A. Bertram and S. Forest. Mechanics of strain gradient materials, 2020.

[16] A. Blinowski, J. Ostrowska-Maciejewska, and J. Rychlewski. Two-dimensional hooke's tensors-isotropic decomposition, effective symmetry criteria. Archives of Mechanics, 48(2):325-345, 1996.

[17] J.-P. Boehler, A. Kirillov, Jr., and E. Onat. On the polynomial invariants of the elasticity tensor. Journal of Elasticity, 34(2):97-110, 1994.

[18] M. Bonnet, R. Cornaggia, and B. Guzina. Microstructural topological sensitivities of the second-order macroscopic model for waves in periodic media. SIAM Journal on Applied Mathematics, 78(4):2057-2082, 2018.

[19] S. Brulé, B. Ungureanu, Y. Achaoui, A. Diatta, R. Aznavourian, T. Antonakakis, R. Craster, S. Enoch, and S. Guenneau. Metamaterial-like transformed urbanism. Innovative Infrastructure Solutions, 2(1):20, 2017.

[20] P. Casal. Capillarité interne en mécanique des millieux continus. Comptes Rendu Mécanique, 256(3), 1961.

[21] Z. Chen, Y. Chen, L. Qi, and W. Zou. Two irreducible functional bases of isotropic invariants of a fourth-order threedimensional symmetric and traceless tensor. Mathematics and Mechanics of Solids, 24(10):3092-3102, 2019.

[22] N. Cordero, S. Forest, and E. Busso. Second strain gradient elasticity of nano-objects. Journal of the Mechanics and Physics of Solids, 97:92-124, 2016.

[23] E. Cosserat and F. Cosserat. Théorie des corps déformables. 1909.

[24] D. Da, J. Yvonnet, L. Xia, M. V. Le, and G. Li. Topology optimization of periodic lattice structures taking into account strain gradient. Computers \& Structures, 210:28-40, 2018.

[25] F. dell'Isola, P. Seppecher, M. Spagnuolo, E. Barchiesi, F. Hild, T. Lekszycki, I. Giorgio, L. Placidi, U. Andreaus, M. Cuomo, et al. Advances in pantographic structures: design, manufacturing, models, experiments and image analyses. Continuum Mechanics and Thermodynamics, 31(4):1231-1282, 2019.

[26] B. Desmorat and N. Auffray. Space of 2D elastic materials: a geometric journey. Continuum Mechanics and Thermodynamics, 31(4):1205-1229, 2019.

[27] B. Desmorat, M. Olive, N. Auffray, R. Desmorat, and B. Kolev. Computation of minimal covariants bases for 2D coupled constitutive laws. arXiv preprint arXiv:2007.01576, 2020.

[28] D. P. DiVincenzo. Dispersive corrections to continuum elastic theory in cubic crystals. Physical Review B, 34(8):5450, 1986.

[29] V. Eremeyev, S. Lurie, Y. Solyaev, and F. dell'Isola. On the well posedness of static boundary value problem within the linear dilatational strain gradient elasticity. Zeitschrift für angewandte Mathematik und Physik, 71(6):1-16, 2020.

[30] V. Eremeyev and W. Pietraszkiewicz. Local symmetry group in the general theory of elastic shells. Journal of Elasticity, 85(2):125-152, 2006.

[31] V. A. Eremeyev. On effective properties of materials at the nano-and microscales considering surface effects. Acta Mechanica, $227(1): 29-42,2016$. 
[32] A. C. Eringen. Mechanics of micromorphic continua. In Mechanics of generalized continua, pages 18-35. Springer, Berlin, Heidelberg, 1968.

[33] A. C. Eringen. Micropolar theory of liquid crystals. In Liquid crystals and ordered fluids, pages 443-474. Springer, Boston, MA., 1978.

[34] S. Forest. Mechanics of generalized continua: construction by homogenizaton. Journal de Physique IV, 8(PR4):Pr4-39, 1998.

[35] S. Forest. Strain gradient elasticity from capillarity to the mechanics of nano-objects. In Mechanics of Strain Gradient Materials, pages 37-70. Springer, 2020.

[36] S. Forte and M. Vianello. Symmetry classes for elasticity tensors. Journal of Elasticity, 43(2):81-108, 1996.

[37] S. Forte and M. Vianello. Symmetry classes and harmonic decomposition for photoelasticity tensors. International Journal of Engineering Science, 35(14):1317 - 1326, 1997.

[38] M. Golubitsky, I. Stewart, and D. Schaeffer. Singularities and Groups in Bifurcation Theory. Vol. II, volume 69 of Applied Mathematical Sciences. Springer-Verlag, New York, 1988.

[39] M. Gotoh. A theory of plastic anisotropy based on a yield function of fourth order (plane stress state)-I. International Journal of Mechanical Sciences, 19(9):505-512, 1977.

[40] A. E. Green and W. Zerna. Theoretical elasticity. Courier Corporation, 1992.

[41] Q.-C. He and Q.-S. Zheng. On the symmetries of 2D elastic and hyperelastic tensors. Journal of elasticity, 43:203-225, 1996.

[42] R. Hedayati, A. Leeflang, and A. Zadpoor. Additively manufactured metallic pentamode meta-materials. Applied Physics Letters, 110(9):091905, 2017.

[43] J. Hutchinson and N. Fleck. Strain gradient plasticity. Advances in Applied Mechanics, 33:295-361, 1997.

[44] L. Jakabčin and P. Seppecher. On periodic homogenization of highly contrasted elastic structures. Journal of the Mechanics and Physics of Solids, 144:104104, 2020.

[45] J. Jerphagnon, D. Chemla, and R. Bonneville. The description of the physical properties of condensed matter using irreducible tensors. Advances in Physics, 27:609-650, 1978.

[46] M. Kadic, T. Bückmann, N. Stenger, M. Thiel, and M. Wegener. On the practicability of pentamode mechanical metamaterials. Applied Physics Letters, 100(19):191901, 2012.

[47] K.-I. Kanatani. Distribution of directional data and fabric tensors. International Journal of Engineering Science, 22(2):149164,1984

[48] W. Koiter. Couple-stresses in the theory of elasticity, I \& II. Philosophical Transactions Of The Royal Society Of London $B, 67: 17-44,1969$.

[49] H. Le Quang and Q.-C. He. The number and types of all possible rotational symmetries for flexoelectric tensors. Proceedings of the Royal Society A, 467(2132):2369-2386, 2011.

[50] G. Maugin. A historical perspective of generalized continuum mechanics. In Mechanics of generalized continua, pages 3-19. Springer, 2011.

[51] G. W. Milton and A. V. Cherkaev. Which elasticity tensors are realizable? Journal of Engineering Materials and Technology, 117(4):483-493, 1995.

[52] R. Mindlin. Micro-structure in linear elasticity. Archive for Rational Mechanics and Analysis, 16(1), 1964.

[53] R. Mindlin. Second gradient of strain and surface-tension in linear elasticity. International Journal of Solids and Structures, 1(4):417-438, 1965.

[54] R. Mindlin and N. Eshel. On first strain-gradient theories in linear elasticity. International Journal of Solids and Structures, 4(1):109-124, 1968.

[55] N. Muskhelishvili. Some basic problems of the mathematical theory of elasticity. Springer Science \& Business Media, 2013.

[56] M. Olive, B. Kolev, and N. Auffray. A minimal integrity basis for the elasticity tensor. Archive for Rational Mechanics and Analysis, 226(1):1-31, 2017.

[57] M. Poncelet, A. Somera, C. Morel, C. Jailin, and N. Auffray. An experimental evidence of the failure of Cauchy elasticity for the overall modeling of a non-centro-symmetric lattice under static loading. International Journal of Solids and Structures, 147:223-237, 2018

[58] G. Racah. Determinazione del numero dei tensori isotropi indipendenti di rango n. Rendiconti della R. Accademia dei Lincei, classe di scienze fisiche, matematiche e naturali, 18:387-389, 1933.

[59] N. Ranaivomiarana. Simultaneous optimization of topology and material anisotropy for aeronautic structures. PhD thesis, Sorbonne Université, 2019.

[60] N. Ranaivomiarana, F.-X. Irisarri, D. Bettebghor, and B. Desmorat. Concurrent optimization of material spatial distribution and material anisotropy repartition for two-dimensional structures. Continuum Mechanics and Thermodynamics, 31(1):133$146,2019$.

[61] G. Rosi and N. Auffray. Anisotropic and dispersive wave propagation within strain-gradient framework. Wave Motion, 63:120-134, 2016.

[62] G. Rosi and N. Auffray. Continuum modelling of frequency dependent acoustic beam focussing and steering in hexagonal lattices. European Journal of Mechanics-A/Solids, 77:103803, 2019.

[63] G. Rosi, L. Placidi, and N. Auffray. On the validity range of strain-gradient elasticity: a mixed static-dynamic identification procedure. European Journal of Mechanics-A/Solids, 69:179-191, 2018.

[64] G. Rosi, P. Ropars, and N. Auffray. New mitigation solution by waves deviation, numerical experiments. In Euronoise, 2018. 
[65] P. Seppecher. Etude des conditions aux limites en théorie du second gradient: cas de la capillarité. Comptes rendus de l'Académie des sciences. Série 2, Mécanique, Physique, Chimie, Sciences de l'univers, Sciences de la Terre, 309(6):497$502,1989$.

[66] G. F. Smith and G. Bao. Isotropic invariants of traceless symmetric tensors of orders three and four. International Journal of Engineering Science, 35(15):1457-1462, 1997.

[67] V. Smyshlyaev and N. Fleck. The role of strain gradients in the grain size effect for polycrystals. Journal of the Mechanics and Physics of Solids, 44(4):465-496, 1996.

[68] A. Spencer. A note on the decomposition of tensors into traceless symmetric tensors. International Journal of Engineering Science, 8:475-481, 1970.

[69] E. Tonti. The mathematical structure of classical and relativistic physics. Springer, 2013.

[70] R. Toupin and B. Bernstein. Sound waves in deformed perfectly elastic materials. acoustoelastic effect. The Journal of the Acoustical Society of America, 33(2):216-225, 1961.

[71] R. A. Toupin. Elastic materials with couple-stresses. Archive for Rational Mechanics and Analysis, 11:385-414, 1962.

[72] D. K. Trinh, R. Janicke, N. Auffray, S. Diebels, and S. Forest. Evaluation of generalized continuum substitution models for heterogeneous materials. International Journal for Multiscale Computational, 10(6), 2012.

[73] P. Vannucci. The polar analysis of a third order piezoelectricity-like plane tensor. International Journal of Solids and Structures, 44:7803-7815, 2007.

[74] G. Verchery. Les invariants des tenseurs d'ordre 4 du type de l'élasticité. In Mechanical Behavior of Anisotropic Solids/Comportment Méchanique des Solides Anisotropes, pages 93-104. Springer, 1982.

[75] F. Vernerey, W. Liu, and B. Moran. Multi-scale micromorphic theory for hierarchical materials. Journal of the Mechanics and Physics of Solids, 55(12):2603-2651, 2007.

[76] M. Vianello. An integrity basis for plane elasticity tensors. Archives of Mechanics, 49(1):197-208, 1997.

[77] K. Wang, Y.-H. Chang, Y. Chen, C. Zhang, and B. Wang. Designable dual-material auxetic metamaterials using threedimensional printing. Materials \& Design, 67:159-164, 2015.

[78] H. Weyl. The classical groups: their invariants and representations, volume 45. Princeton University Press, 1946.

[79] Q.-S. Zheng. Theory of representations for tensor functions - a unified invariant approach to constitutive equations. Applied Mechanics Reviews, 47:545-587, 1994.

[80] Q.-S. Zheng and W.-N. Zou. Irreducible decompositions of physical tensors of high orders. Journal of Engineering Mathematics, 37(1-3):273-288, 2000.

[81] H. Zhu. Size-dependent elastic properties of micro-and nano-honeycombs. Journal of the Mechanics and Physics of Solids, $58(5): 696-709,2010$

[82] W.-N. Zou, Q.-S. Zheng, D.-X. Du, and J. Rychlewski. Orthogonal irreducible decompositions of tensors of high orders. Mathematics and Mechanics of Solids, 6(3):249-267, 2001.

(Nicolas Auffray) Univ Gustave Eiffel, CNRS, MSMe UMR 8208, F-77454 Marne-la-Vallée, France

Email address: nicolas.auffray@univ-eiffel.fr

(Houssam Abdoul-Anziz) Univ Gustave Eiffel, CNRS, MSME UMR 8208, F-77454 Marne-la-Vallée, France

Email address: houssam.abdoulanziz@u-pem.fr

(Boris Desmorat) Sorbonne Université, CNRS, Institut Jean Le Rond D'Alembert, UMR 7190, 75005 Paris, FRANCE

Email address: boris.desmorat@sorbonne-universite.fr 